\title{
Bees of the genus Lasioglossum (Hymenoptera: Halictidae) from Greater Puerto Rico, West Indies
}

\author{
Jason GIBBS \\ Department of Entomology, University of Manitoba, 12 Dafoe Road, \\ Winnipeg, R3T 2N2 Manitoba, Canada. \\ Email: jason.gibbs@umanitoba.ca \\ urn:1sid:zoobank.org:author:BA42A49F-3EBC-4679-8F03-A58E798106B1
}

\begin{abstract}
The species of Lasioglossum from Greater Puerto Rico are reviewed. Nine species are recognized, including five new species described herein: Lasioglossum (Dialictus) genaroi sp. nov., L. (D.) dispersum sp. nov., L. (D.) enatum sp. nov., $L$. (D.) monense sp. nov. and $L$. (D.) amona sp. nov. The latter two are known only from Mona Island. Keys and images are provided to assist in identification. Details of nesting biology, floral hosts and distribution are provided where available. Three species, L. (D.) parvum (Cresson, 1865), L. (D.) busckiellum (Cockerell, 1915), and L. (D.) mestrei (Baker, 1906) are removed from the list of species for Puerto Rico. Details on their revised distribution are provided. Three new records for Haiti, L. (D.) gundlachii (Baker, 1906), L. (D.) ferrerii (Baker, 1906) and L. (D.) busckiellum are documented. Notes on other species in the Greater Antilles are provided, including the synonymy of Lasioglossum bruesi (Cockerell, 1912) and L. jamaicae (Ellis, 1914) under L. gemmatum (Smith, 1853).
\end{abstract}

Keywords. Apoidea, distribution, new species, synonymy, taxonomy.

Gibbs J. 2018. Bees of the genus Lasioglossum (Hymenoptera: Halictidae) from Greater Puerto Rico, West Indies. European Journal of Taxonomy 400: 1-57. https://doi.org/10.5852/ejt.2018.400

\section{Introduction}

Halictid bees are abundant members of most terrestrial ecosystems (Michener 1979; Eickwort 1988). In the Greater Antilles group of Caribbean Islands, members of the family Halictidae make up nearly one third of bee species (Alayo 1973, 1976; Raw 1985; Eickwort 1988; Ascher \& Pickering 2016). The halictid genus Lasioglossum Curtis, 1933 is the most species rich (Ascher \& Pickering 2016) and behaviourally diverse group of bees globally (Michener 1974, 2007; Wcislo 1997; Yanega 1997; Schwarz et al. 2007). Twenty-one described species of Lasioglossum are currently recognized from the Greater Antilles. These have been described over a period of more than 160 years (Smith 1853; Cresson 1865; Ashmead 1896; Baker 1906; Cockerell 1912, 1915; Ellis 1914; Sandhouse 1924; Graenicher 1927; Engel 2001b; Genaro 2001, 2016). Undescribed species of Lasioglossum are also known to occur on islands across the Greater Antilles (Alayo 1976; J. Gibbs, unpublished data; J.A. Genaro, in litt.). 
Two subgenera of Lasioglossum are known from the West Indies: L. (Dialictus) Robertson, 1902 and L. (Habralictellus) Moure \& Hurd, 1982. Lasioglossum (Dialictus) is globally distributed and includes hundreds of species (Michener 2007; Gibbs et al. 2012b; Ascher \& Pickering 2016; Gibbs 2016), while L. (Habralictellus) is endemic to the West Indies (Eickwort 1988; Gibbs 2016), with ten described species (Engel 2001b; Genaro 2001, 2016; Gibbs 2016). Lasioglossum (Dialictus) are dull metallic bees, while L. (Habralictellus) are typically bright iridescent green or blue, often similar in colour to some augochlorine bees (Michener 2007). It has been presumed that L. (Habralictellus) is derived from and therefore should be synonymized with L. (Dialictus) (Eickwort 1988; Michener 2007; Genaro \& Franz 2008; Gibbs 2011). However, preliminary analyses of molecular data from multiple loci suggest this synonymy may be premature (J. Gibbs, unpublished data; see also Fig. 1). The relationship of L. (Habralictellus) to L. (Dialictus) and even the monophyly of L. (Habralictellus) remain uncertain. Eickwort (1988) recognized three species groups (A, B, and C) within L. (Habralictellus) based on the morphological variation within that group. These apparently correspond to groups represented by L. rufopanticis (Engel, 2001), L. auratum (Ashmead, 1900), and L. adriani Genaro, 2001. These species groups differ in body size and shape, surface sculpturing and wing venation (Engel 2001b; Genaro 2001; Gibbs 2016). Despite these differences, there are undescribed species that might represent intermediate forms between these species groups that could serve to unite them (J. Gibbs, unpublished data).

Eickwort (1988) recognized eleven species groups of Lasioglossum from the West Indies, summarized available knowledge of West Indian halictid bee biogeography and published brief biological notes on some species from Puerto Rico and the Bahamas. In a survey of the bees of Greater Puerto Rico (including the US and British Virgin Islands), Genaro \& Franz (2008) recognized seven species of Lasioglossum (all as subgenus Dialictus): L. (Dialictus) busckiellum (Cockerell, 1915), L. (D.) ferrerii (Baker, 1906); L. (D.) gundlachii (Baker, 1906), L. (D.) mestrei (Baker, 1906), L. (D. ) parvum (Cresson, 1865), L. (Habralictellus) eickwortellum (Engel, 2001) and L. (H.) rufopanticis. Lasioglossum busckiellum was described from the Dominican Republic (Cockerell 1915); the remaining four L. (Dialictus) were described from Cuba (Baker 1906; Cresson 1865); L. rufopanticis was described from the British Virgin Islands and four Puerto Rican paratypes including Mona Island (Engel 2001b); and L. eickwortellum was known only from the Puerto Rican holotype (Engel 2001b; Genaro \& Franz 2008). As part of ongoing revisionary studies of North American Lasioglossum (e.g., Gibbs 2010b, 2011, 2016; Gibbs et al. 2013), and to make names available for ecological studies of Puerto Rican pollinators (S. Prado, unpublished data), the species of Lasioglossum occurring in Greater Puerto Rico (sensu Thomas \& Joglar 1996) are reviewed and five new species described. Three species are removed from list of Puerto Rican bees. Extra-limital records are provided for those species with broader ranges and species removed from the Puerto Rican fauna.

\section{Material and methods}

West Indian halictid bee specimens have been examined from the following institutions:
AMNH $=$ American Museum of Natural History, New York, New York
ANSP = Academy of Natural Sciences of Drexler University, Philadelphia, Pennsylvania
BBSL = Bee Biology and Systematics Laboratory, Logan, Utah
$\mathrm{CMNH}=$ Carnegie Museum of Natural History, Pittsburgh, Pennsylvania
CUIC $=$ Cornell University Insect Collection, Ithaca, New York
FSCA = Florida State Collection of Arthropods, Gainesville, Florida
JBWM = J.B. Wallis/R.E. Roughley Museum of Entomology, Winnipeg, Manitoba
LACM = Natural History Museum of Los Angeles County, Los Angeles, California
$\mathrm{NCSU}=$ North Carolina State University, Raleigh, North Carolina 


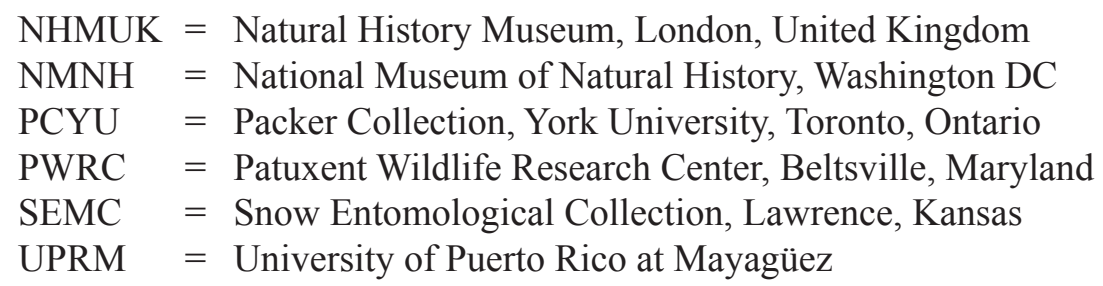

Additional specimens in the care of Julio Genaro (either in his personal collection or on loan) were also examined with his permission (JAGC). Type material for all species of Lasioglossum from the Greater Antilles has been examined, with the exception of L. albitarsatum (Ashmead, 1896), the type series of which is evidently lost (Moure 2007). Attempts to locate the holotype specimen at the three most likely depositories (NMNH, NHMUK and Iowa State Insect Collection) were unsuccessful.

The higher classification of bees is based on Michener (2007), except for the genus Lasioglossum, which follows Gibbs et al. (2013) and Gibbs (2016). Lasioglossum (Habralictellus) is recognized as a valid subgenus separate from $L$. (Dialictus) based on unpublished molecular phylogenetic results (J. Gibbs, unpublished data) and following Gibbs (2016). Species descriptions follow a format used in other recent papers on Lasioglossum (Gibbs 2010b, 2011; Gibbs et al. 2013). Terminology for structures and surface sculpturing follows Michener (2007), Engel (2001a), Gibbs (2010b), Gibbs et al. (2013) and Harris (1979). Puncture density is given in terms of puncture diameter (pd) relative to interspace distance between punctures (i). Metasomal terga (T), sterna (S) and antennal flagellomeres (F) are indicated by their abbreviation followed by the relevant number. Measurements were taken using an ocular micrometre in a Nikon SMZ25 at 40-120× magnification. Maps and statistics were done in $\mathrm{R}$ version 3.2.2 (R Core Team 2015). Maps were created using the $\mathrm{R}$ packages maptools (Bivand \& Lewin-Koh 2017) and ggplot2 (Wickham 2009 and data available from https://catalog.data.gov).

DNA barcodes were generated for a subset of specimens using the primers LepF1 and LepR1 (Hebert et al. 2004) at the Biodiversity Institute of Ontario (Guelph University) following protocols described elsewhere (Gibbs 2009a) (Table 1). Sequences were uploaded to the Barcode of Life Data Systems (www.boldsystems.org) (Ratnasingham \& Hebert 2007) and submitted to GenBank (accession numbers not yet available). Sequences were downloaded and realigned using Muscle (Edgar 2004) to remove unnecessary gaps. A maximum likelihood phylogeny was generated using an online tool (Dereeper et al. 2008) to illustrate the sequence differences between species. Only sequences with greater than $600 \mathrm{bp}$ were included. A small number of additional taxa were included for comparison using publicly available DNA barcode sequences (Gibbs 2009a; Gibbs et al. 2012a, 2013). Two members of the subgenus L. (Hemihalictus) Cockerell, 1897 were included and L. (Evylaeus) cinctipes (Provancher, 1888) was used to root the tree.

\section{Results}

\section{Phylogeny}

Nineteen DNA barcode sequences greater than $600 \mathrm{bp}$ in length were generated from Puerto Rican Lasioglossum (Table 1). Due to limited fresh material and Wolbachia amplifications, no sequences are available for L. eickwortellum or L. ferrerii. Cuban individuals of L. mestrei and L. parvum were also sequenced. A maximum likelihood phylogeny shows discrete, well-supported clades (branch support $>0.95$ ) or distinct branches for five species of Lasioglossum in Puerto Rico (Fig. 1). Puerto Rican specimens in the $L$. parvum species complex are a distinct clade from other $L$. parvum group species $-L$. lepidii (Graenicher, 1927), L. surianae (Mitchell, 1960) and L. parvum - that were included in the analysis. Lasioglossum rufopanticis appears more closely related to members of L. (Hemihalictus), but this analysis is insufficient to resolve its phylogenetic position. 


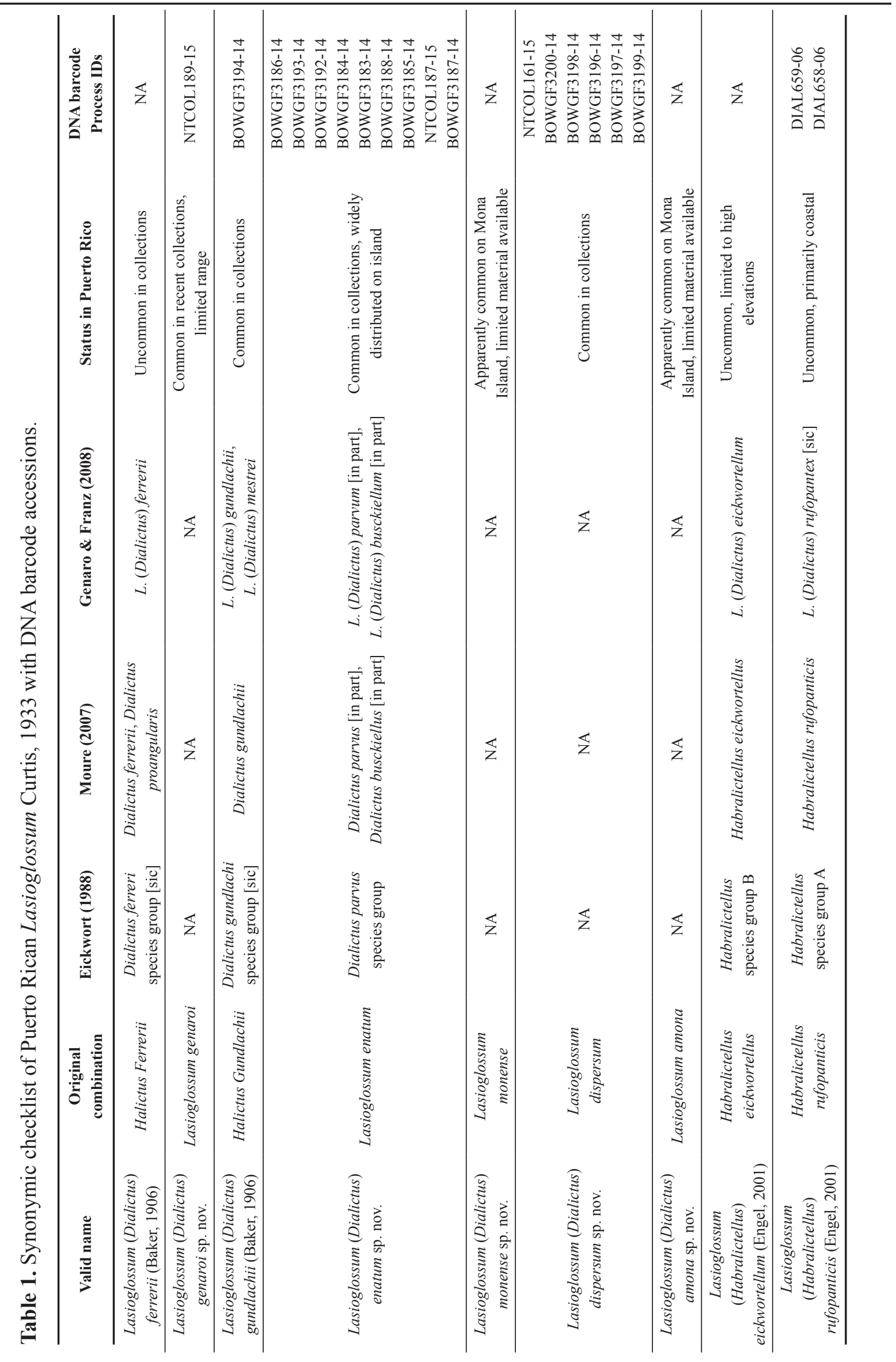


Puerto Rican species of Lasioglossum

Class Hexapoda Blainville, 1816

Order Hymenoptera Linnaeus, 1758

Family Halictidae Thomson, 1869

Tribe Halictini Thomson, 1869

Genus Lasioglossum Curtis, 1933

Subgenus Dialictus Robertson, 1902

Paralictus Robertson, 1901: 229 (type species: Halictus cephalicus Robertson, 1892 (= Halictus cephalotes Dalla Torre, 1896), by original designation).

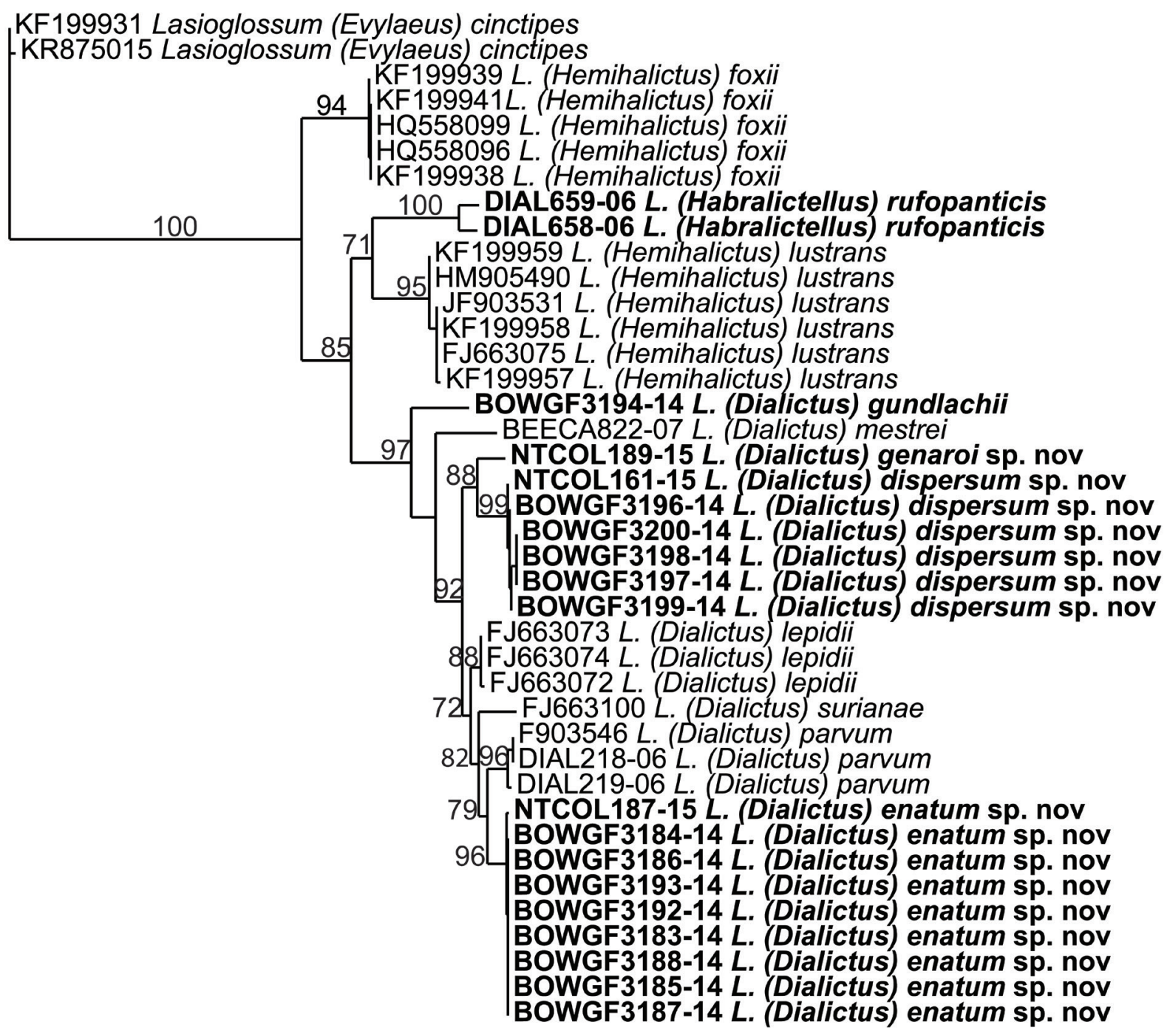

0.4

Fig. 1. Maximum-likelihood phylogeny of DNA barcode sequences of Puerto Rican Lasioglossum species (bold text) including extra-limital taxa for reference. Terminals include the species name with the GenBank accession or BOLD Process ID. Support values are not shown for intraspecific nodes. 
Dialictus Robertson, 1902a: 48 (type species: Halictus anomalus Robertson, 1892, by original designation and monotypy; given priority over Paralictus Robertson, 1901 (International Commission of Zoological Nomenclature 1997)).

Chloralictus Robertson, 1902b: 248 (type species: Halictus cressonii Robertson, 1890, by original designation; given priority over Paralictus Robertson, 1901 (International Commission of Zoological Nomenclature 1997)).

Halictus (Gastrolictus) Ducke, 1902: 102 (type species: Halictus osmioides Ducke, 1902, by monotypy).

Halictomorpha Schrottky, 1911: 81 (type species: Halictomorpha phaedra Schrottky, 1911, by original designation).

Rhynchalictus Moure, 1947: 5 (type species: Rhynchalictus rostratus Moure, 1947, by original designation).

Halictus (Smeathhalictus) Warncke, 1975: 88 (type species: Melitta smeathmanella Kirby, 1802, by original designation).

Lasioglossum (Afrodialictus) Pauly, 1984: 142 (type species: Halictus bellulus Vachal, 1909, by original designation).

Gnathalictus Moure, 2001: 493 (type species: Gnathalictus capitatus Moure, 2001, by original designation).

Evylaeus (Viridihalictus) Pesenko, 2007: 25 (type species: Halictus viridis Brullé, 1840, by original designation).

Evylaeus (Glauchalictus) Pesenko, 2007: 26 (type species: Halictus problematicus Blüthgen, 1823, by original designation).

Evylaeus (Virenshalictus) Pesenko, 2007: 26 (type species: Hylaeus virens Erichson, 1835, by original designation).

Evylaeus (Loethalictus) Pesenko, 2007: 26 (type species: Halictus loetus Brullé, 1840, by original designation).

Evylaeus (Aerathalictus) Pesenko, 2007: 27 (type species: Melitta aerata Kirby, 1802, by original designation).

Lasioglossum (Dialictus) dispersum sp. nov. urn:1sid:zoobank.org:act:36BD3C86-C7F7-4B36-B542-2A5190FE1190

Figs $2-3,4 \mathrm{~B}, 5$

\section{Diagnosis}

Both sexes of $L$. dispersum sp. nov. can be recognized by the combination of tegula oval without extended posterior angle, mesepisternum punctate and metasoma brown. The female has a distinct oblique propodeal carina, T2-T3 basolaterally with appressed tomentum, and T2-T3 apical impressed areas distinctly, but finely punctate. In size and sculpturing, $L$. dispersum sp. nov. is similar to members of the L. parvum species complex, but can be recognized by the unmodified tegula. Females of L. enatum sp. nov. have finer mesoscutal punctures and the T2-T3 apical impressed areas impunctate. Lasioglossum genaroi $\mathrm{sp}$. nov. is similar, but has rugose to reticulate-rugose mesepisternal sculpturing and typically a larger body size, which in females is significantly larger on average $(\mathrm{t}=3.2, \mathrm{df}=17.6$, $p=0.005$ ), and it has a darker blue colour. Lasioglossum amona sp. nov. is most similar, differing only in the female having a paler green integument, a honey-coloured tegula, a smoother mesepisternum and a less distinct oblique propodeal carina.

\section{Etymology}

The specific epithet is taken from the Latin for "scattered, dispersed", which is a reference to the ability of this species and its ancestors to colonize islands in the Caribbean. The Latin dispersum is a participle in the nominative neuter singular. 
GIBBS J., The bee genus Lasioglossum of Greater Puerto Rico

\section{Material examined}

Holotype

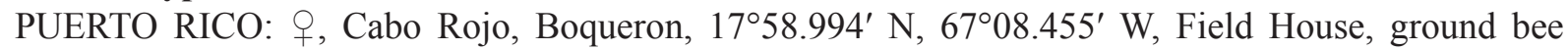
bowl, 12-16 May 2014, S.G. Prado leg. (JBWM). [Original label: USA, PR Boqueron N1758.994' W67 ${ }^{\circ} 08.455^{\prime}$, Field House, ground bee bowl, 12-16 May 2014, S.G. Prado leg., \#49 // HOLOTYPE Lasioglossum (Dialictus) dispersum Gibbs.]

\section{Paratypes}

PUERTO RICO: Barceloneta: 1 , [no locality], ex rose, 21 Feb. 1933, Anderson, Mills and Faxon leg. (NMNH); 1 q, [no locality], 15 Nov. 1932, Anderson, Faxon and Mills leg. (NMNH). - Bayamón: 1 ồ, [no locality], ex milkweed, 2 Jun.1934, Anderson and Lesesne leg. (NMNH). - Cabo Rojo: 1 q, Boqueron, Cabo Rojo National Wildlife Refuge, USFWS, $17^{\circ} 58.816^{\prime} \mathrm{N}, 67^{\circ} 10.231^{\prime} \mathrm{W}$, ground bee bowl, 20-28 May 2014, S.G. Prado leg. (NCSU); 2 우, same collection data as preceding, ground bee bowl, 27 May-13 Jun. 2014 (NCSU);1 , same collection data as preceding, ground bee bowl, 5 Jun. 2014 (NCSU); 1 + , 1 $\hat{\text { }}$, same locality as preceding, Malaise trap, 27 May-13 Jun. 2014, S.G. Prado leg. (NCSU); 1 , same locality as preceding, net, 29 May 2014, S.G. Prado leg. (NCSU); 5 q $q$, same collection data as preceding, 3 Jun. 2014 (NCSU); 1 , 1 ${ }^{\lambda}$, same collection data as preceding, 5 Jun. 2014 (NCSU); 35 우, Las Palmas, Field House, $17^{\circ} 58.994^{\prime}$ N, $67^{\circ} 08.455^{\prime} \mathrm{W}$, ground bee bowl, 12-16 May 2014, S.G. Prado leg. (JBWM); 1 + , Los Morrillos State Forest, near the lighthouse, $17^{\circ} 56^{\prime} 08^{\prime \prime} \mathrm{N}$, 67¹1'35" W, 20 May 2010, E. Castro-Feliu leg. (UPRM). - Cataño: 1 Oૈ, 1932, C.F. Dolman leg. (AMNH). - Culebra: 1 \%, Isla Culebra, Punta Flamenco, 7 Nov. 2008, J.A. Genaro leg. (JAGC). Fajardo: 1 q, Cabezas de San Juan, Oct. 1997, J.A. Genaro leg. (JAGC). - Guánica: 1 ồ stylopized, Bosque Estatal de Guánica, $17.84^{\circ} \mathrm{N}, 66.86^{\circ} \mathrm{W}$, light trap, 5 Jun. 1996, M. Canals leg. (LACM); 1 ð, same collection data as preceding, 4 Jul. 1997 (LACM); 1 ㅇ, same collection data as preceding, $20 \mathrm{Jul}$. 1998 (LACM); 1 ㅇ, same collection data as preceding, 18 Aug. 1997 (LACM); 1 + , same collection data as preceding, 24 Aug. 1996 (LACM); 3 우, same collection data as preceding, 15 Sep. 1996 (LACM); 19 , same collection data as preceding, 19 Sep. 1996 (LACM); 1 \%, Bosque Estatal de Guánica, $17.961^{\circ} \mathrm{N}$, 66.847 ${ }^{\circ}$ W, 75 ft a.s.1., Malaise trap, 19-25 Sep. 1998, R.R. Snelling leg. (LACM); 5 우, Bosque Estatal de Guánica, $17.967^{\circ}$ N, $66.878^{\circ}$ W, $360 \mathrm{ft}$ a.s.l., Malaise trap, 19-27 Sep. 1998, R.R. Snelling

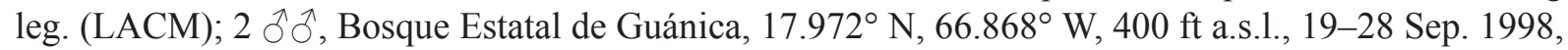
R.R. Snelling leg. (LACM); 1 \%, Bosque Estatal de Guánica, $17.84^{\circ} \mathrm{N}, 66.86^{\circ} \mathrm{W}$, light trap, 23 Sep. 1997, M. Canals leg. (LACM); 1 ㅇ, same collection data as preceding, 29 Sep. 1996 (LACM); 1 ô, same collection data as preceding, 16 Oct. 1996 (LACM); 1 q, same collection data as preceding, 4 Nov. 1996 (LACM); 1 J , same collection data as preceding, 21 Dec. 1996 (LACM). - Guayanilla: 1 9, May 2006, J.A. Genaro leg. (JAGC); 1 \&, Ventana, ex Waltheria indica L., Feb. 2009, J.A. Genaro leg. (JAGC). Maricao: $1 \partial^{\jmath}$, Damiani, shade, $18^{\circ} 11.862^{\prime} \mathrm{N}, 66^{\circ} 56.347^{\prime} \mathrm{W}$, net (9:50-11:50), 18 Jun. 2014, S.G. Prado leg. (NCSU). - Mayagüiez: 1 , Carr: 108, km 8.0, 22 Aug. 1969, C.G. Moore leg. (JAGC). - Ponce: 1 , , A20, $18^{\circ} 02.275^{\prime} \mathrm{N}, 66^{\circ} 38.585^{\prime} \mathrm{W}$, Malaise trap, 17 Jun.-15 Jul. 2014, S.G. Prado leg. (NCSU); 2 우, same collection data as preceding, 12 Aug.-9 Sep. 2014 (NCSU); 1 , same collection data as preceding, 9 Sep.-7 Oct. 2014 (NCSU); 1 q, same collection data as preceding, 4 Nov.-2 Dec. 2014 (NCSU); 1 \%, same locality as preceding, ground bee bowl, 17 Jun.-15 Jul. 2014, S.G. Prado leg. (NCSU); 1 q, same collection data as preceding, 15 Jul.-12 Aug. 2014 (NCSU); 1 , Costa Oeste, Punta Cucharas, 12 Sep.

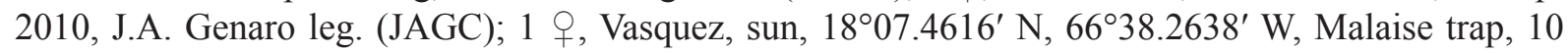
Feb.-10 Mar. 2015, S.G. Prado leg. (NCSU); 1 , same collection data as preceding, 15 Jul.-12 Aug. 2014 (NCSU); 1 \%, same locality as preceding, elevated bee bowl, 9 Sep.-7 Oct. 2014, S.G. Prado leg. (NCSU); 1 ' , Vasquez, shade, $18^{\circ} 07.4628^{\prime} \mathrm{N}, 66^{\circ} 38.1882^{\prime} \mathrm{W}$, ground bee bowl, 9 Sep. -7 Oct. 2014 , S.G. Prado leg. (NCSU); 1 , same locality as preceding, elevated bee bowl, 2 Dec. 2014-13 Jan. 2014, S.G. Prado leg. (NCSU). - Salinas: 1 , , [no locality], 23 Mar. 1929, S.T. Danforth leg. (NMNH). - San Juan: 2 ठึ, 13 Sep. 1939 (NMNH); 1 त, 28 Jun. 1936, W.A. Hoffman leg. (NMNH). - Santa Isabel: 19 , Cohen, $17^{\circ} 59.737^{\prime} \mathrm{N}, 66^{\circ} 25.451^{\prime} \mathrm{W}, 24 \mathrm{Apr} .2013$, S. Prado leg. (NCSU); 3 q $q$, Gomez, $17^{\circ} 59.705^{\prime} \mathrm{N}$, 
66 25.063' W, 23 Jan. 2013, S. Prado leg. (NCSU); 5 우, same collection data as preceding, 13 Mar.

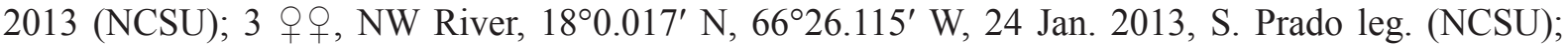
15 우우, same collection data as preceding, 13 Mar. 2013 (NCSU: CCDB-22788 B02); 11 우오, same collection data as preceding, 24 Apr. 2013 (NCSU); 1 , same collection data as preceding, 26 Apr. 2013 (NCSU); 1 ㅇ, Portolain, $17^{\circ} 58.632^{\prime}$ N, 66²3.096' W, 29 Jan. 2013, S. Prado leg. (NCSU: CCDB22788 B05); 5 우우, SE River, $17^{\circ} 58.547^{\prime}$ N, 66 $6^{\circ} 25.063^{\prime}$ W, 22 Jan. 2013, S. Prado leg. (NCSU); 2 우우, same collection data as preceding, 28 Jan. 2013 (NCSU); 9 우, 1 § , same collection data as preceding, 13 Mar. 2013 (NCSU); 4 우, same collection data as preceding, 15 Mar. 2013 (NCSU); 2 우, same

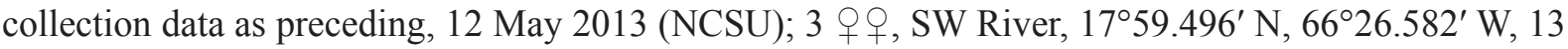
Mar. 2013, S. Prado leg. (NCSU); 2 우, same collection data as preceding, 15 Mar. 2013 (NCSU); 4 우, same collection data as preceding, 24 Apr. 2013 (NCSU); 2 우, same collection data as preceding,
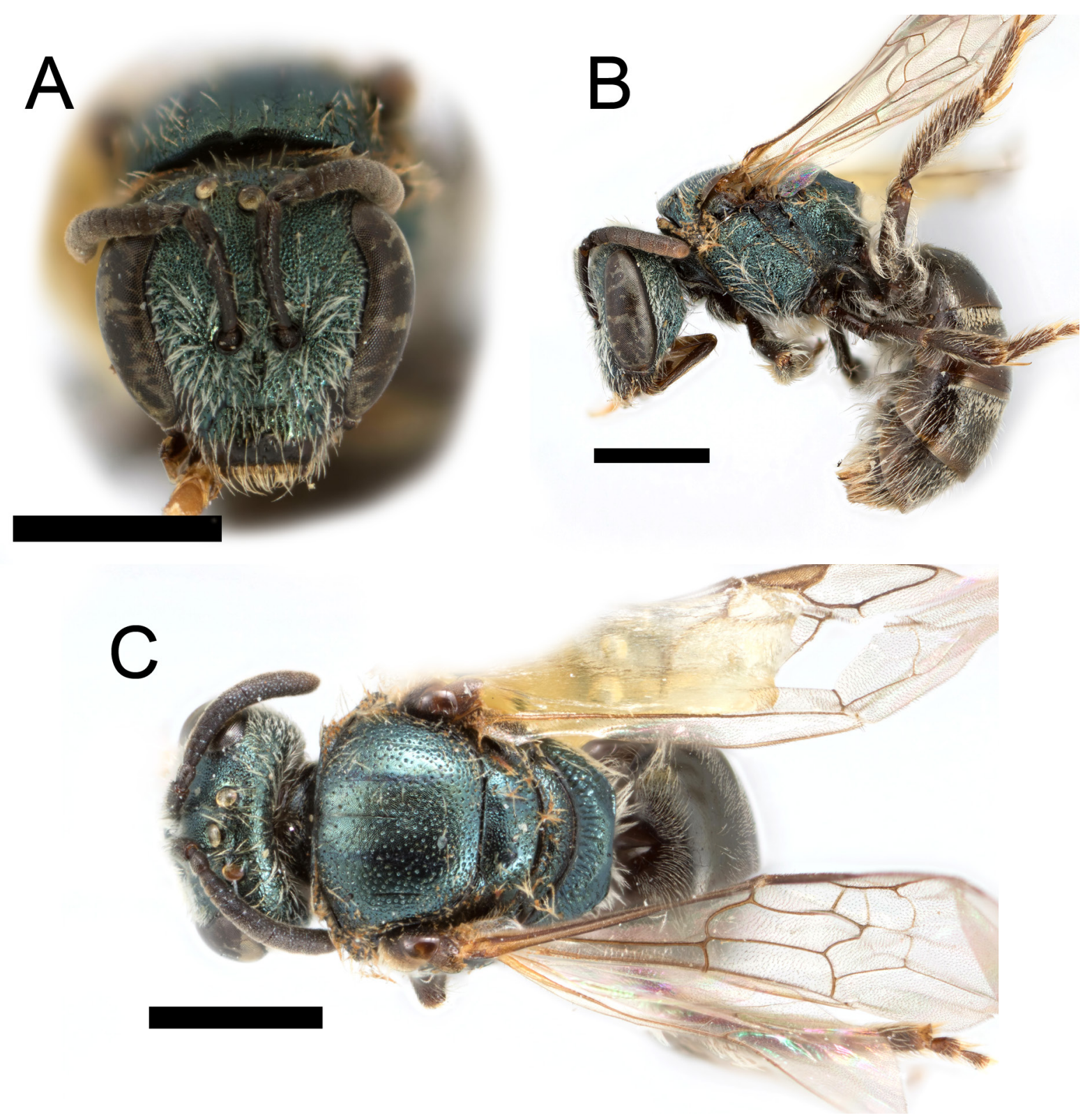

Fig. 2. Lasioglossum dispersum sp. nov., ${ }^{+}$. A. Face. B. Lateral habitus. C. Dorsal habitus. Scale bars $=1 \mathrm{~mm}$. 
26 Apr. 2013 (NCSU). - Yauco: 4 우, A7 Church site, $18^{\circ} 01.830^{\prime} \mathrm{N}, 66^{\circ} 53.042^{\prime} \mathrm{W}$, ground bee bowl, 23 May-20 Jun. 2014, S.G. Prado leg. (NCSU); 4 우, same collection data as preceding, 20 Jun. -18 Jul. 2014 (NCSU); 2 우, same collection data as preceding, 18 Jul.-15 Aug. 2014 (NCSU); 1 , same collection data as preceding, 15 Aug. -12 Sep. 2014 (NCSU); 4 우, same locality as preceding, Malaise trap, Jun.-Jul. 2014, S.G. Prado leg. (NCSU); 6 우, Vega, $18^{\circ} 07.724^{\prime}$ N, 66 $53.386^{\prime}$ W, Malaise trap, 20 Jun.-18 Jul. 2014, S.G. Prado leg. (NCSU); 2 9 , s same collection data as preceding, 18 Jul.-15 Aug. 2014 (NCSU); 4 웅, same collection data as preceding, 15 Aug.-12 Sep. 2014 (NCSU); 1 ㅇ, same collection data as preceding, 12 Sep.-10 Oct. 2014 (NCSU); 1 q, same locality as preceding, elevated bee bowl, 20 Jun.-18 Jul. 2014, S.G. Prado leg. (NCSU); 1 ㅇ, same collection data as preceding, 5 Nov. -5 Dec. 2014 (NCSU); 1 ㅇ, Villa Cecilia, $18^{\circ} 08.371^{\prime}$ N, 66 $49.230^{\prime}$ W, elevated bee bowl, 12 Feb.-12 Mar. 2015, S.G. Prado leg. (NCSU).
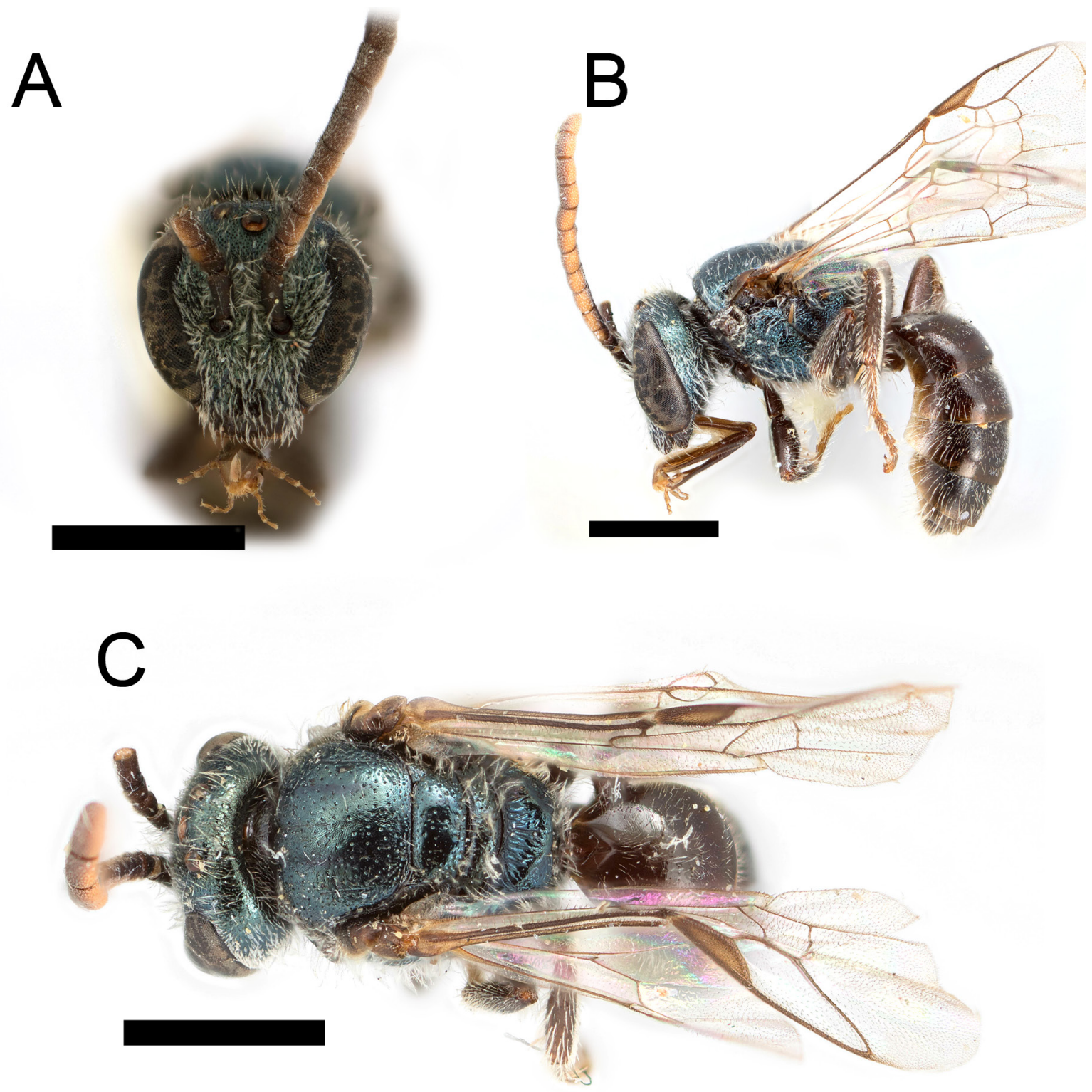

Fig. 3. Lasioglossum dispersum sp. nov., §̂. A. Face. B. Lateral habitus. C. Dorsal habitus. Scale bars $=1 \mathrm{~mm}$. 
US VIRGIN ISLANDS: St. Croix, Anna's Hope Village: 8 q $q$, ArtFarm, $17^{\circ} 42.982^{\prime} \mathrm{N}, 64^{\circ} 41.701^{\prime} \mathrm{W}$, bowls, 20 Mar. 2013, S. Prado leg. (NCSU); 8 + , same collection data as preceding, 18 Apr. 2013 (NCSU); 2 q $ᄋ$, same locality as preceding, net, 20 Mar. 2013, S. Prado leg. (NCSU: CCDB-22788 B04). - Christiansted: 4 ${ }_{+}, 3 \partial^{\lambda}{ }^{\lambda}$, [no locality], 1940, H.A. Beatty leg. (NMNH). - Northcentral: 2 우오, Dale, $17^{\circ} 44.263^{\prime} \mathrm{N}, 64^{\circ} 47.920^{\prime} \mathrm{W}, 18$ Mar. 2013, S. Prado leg. (NCSU); 1 옹 same locality as preceding, bowl, 6 Jun. 2013, S. Prado leg. (NCSU: CCDB-22788 B06); 5 우우, Liburd, 1743.186' N,

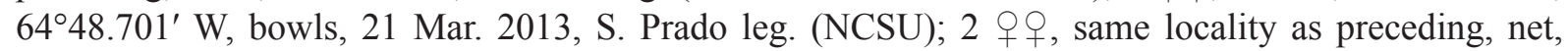

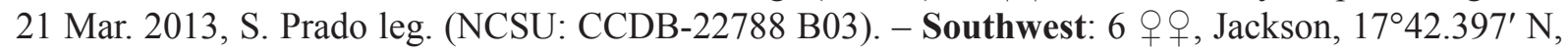

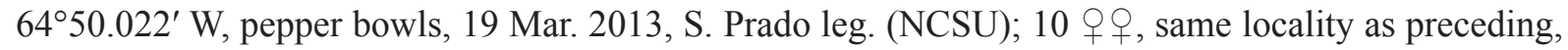
pumpkin bowls, 19 Mar. 2013, S. Prado leg. (NCSU). - Buck Island Reef National Monument: 26 $\circ$, 2 ô $^{\wedge}, 17.7858^{\circ} \mathrm{N}, 64.6269^{\circ} \mathrm{W}, 13 \mathrm{Aug} .2010$, I. Lundgren leg. (PWRC); 1 q, $17.7802^{\circ} \mathrm{N}, 64.7616^{\circ} \mathrm{W}$, 13 Aug. 2010, I. Lundgren leg. (PWRC).

BRITISH VIRGIN ISLANDS: Anegada Island: 1 ð̊, E end, 17 Oct. 1992, R.R. Snelling leg. (LACM). - Guana Island: 1 , The Flat, Malaise trap, 29 Sep. 2006, B.D. Valentine family leg. (AMNH); 1 , flowers, 6 Oct. 2009, D. Valentine leg. (AMNH); 1 , same collection data as preceding, 8 Oct. 2009 (AMNH); 1 + , 5-sand pit, Malaise trap, 21 Oct. 2001, B. Valentine, leg. (AMNH); 1 , flowers, 16 Oct. 2009, B.D. Valentine family leg. (AMNH); 1 , flowers, 17 Oct. 2009, S. Valentine-Cooper leg.

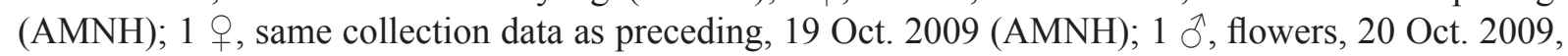
S. Valentine-Cooper leg. (AMNH); 4 우, hotel area, 5 Oct. 1991, R.R. Snelling leg. (LACM); 2 우,

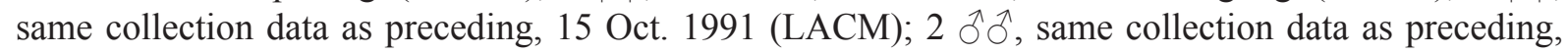
19 Oct. 1991 (LACM); 1 o, same collection data as preceding, 21 Oct. 1991 (LACM); 2 q $q$, same collection data as preceding, 23 Oct. 1991 (LACM); 2 +q, same collection data as preceding, 26-27 Oct. 1992 (LACM); 2 +q, 1 ô, hotel area, ex Antigonon leptopus, 11 Oct. 1992, R.R. Snelling leg. (LACM); 4 우, hotel area, ex Capparis cynophallophora, 13-14 Oct. 1992, R.R. Snelling leg. (LACM); 3 우, same collection data as preceding, 17-19 Oct. 1992 (LACM); 2 우, North Beach, $18^{\circ} 29^{\prime}$ N, $64^{\circ} 34^{\prime}$ W, ex Jacquemontia pentantha, 8 Oct. 1992, R.R. Snelling leg. (LACM); 1 q, same collection data as preceding, 9 Oct. 1992 (LACM); 1 +, North Beach, 28 Oct. 1992, R.R. Snelling leg.

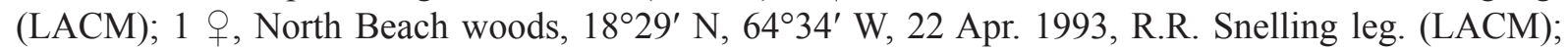
1 , plantation area, $18^{\circ} 29^{\prime}$ N, 643' $4^{\prime}$ W, 23 Apr. 1993, R.R. Snelling leg. (LACM); 1 , plantation area, $18^{\circ} 28.7^{\prime}$ N, $64^{\circ} 34.4^{\prime}$ W, 28 Jun. 1993, R.R. Snelling leg. (LACM); 1 ㅇ, plantation area, ex Jacquemontia pentantha, 18 Oct. 1992, R.R. Snelling leg. (LACM); 1 + , plantation area, 25 Oct. 1992, R.R. Snelling leg. (LACM); $1 \delta$, Quail Dove Ghut, Malaise trap, Jan. 2002, W.-P. Liao leg. (AMNH); 1 q, White Beach, $18^{\circ} 29^{\prime} \mathrm{N}, 64^{\circ} 34^{\prime}$ W, 5 Jul. 1993, R.R. Snelling leg. (LACM); 1 ㅇ, White Beach flats, $18^{\circ} 28.7^{\prime} \mathrm{N}$, 64³4' W, Malaise trap, 9 Jul. 1993, R.R. Snelling leg. (LACM). - Necker Island: 1 q, 21 Jul. 1987, S.E. Miller leg. (LACM).

\section{Description}

\section{Female}

Measurements. Head length: $1.28-1.48 \mathrm{~mm}(\overline{\mathrm{x}}=1.40 \mathrm{~mm}, \mathrm{n}=10)$; head width: $1.34-1.66 \mathrm{~mm}$ $(\overline{\mathrm{x}}=1.50 \mathrm{~mm}, \mathrm{n}=10)$; intertegular distance: $0.94-1.21 \mathrm{~mm}(\overline{\mathrm{x}}=1.12 \mathrm{~mm}, \mathrm{n}=10)$.

Colouration. Head and mesosoma dull metallic golden green, except as follows. Labrum brown. Mandible brown with red apex. Clypeus distal-half dark brown. Supraclypeal area bronze. Antenna dark brown, F9-F10 with ventral surface dark reddish brown. Tegula dark reddish brown. Wing membrane hyaline with dark setae, venation and pterostigma brown. Legs dark brown, except medio- and distitarsi reddish brown. Metasomal terga brown.

Pubescence. Dull white. Relatively sparse erect setae throughout, without tomentum, except narrow basolateral patches of T2-T3 and basally on T4. T1 with complete fan of appressed setae on anterior 
surface. T2 without apical fimbriae, T3-T4 with only sparse fine setae on apical impressed areas. Scopa well developed on hind leg and metasomal sterna.

SuRfaCE SCULPTURE. Face imbricate, punctation moderately coarse, finer on frons. Clypeus punctation sparse $(i=1-2.5 \mathrm{pd})$, surface smooth distally $(\mathrm{i}=2-3 \mathrm{pd})$, supraclypeal area with punctures moderately dense $(\mathrm{i}=1-1.5 \mathrm{pd})$, and lower paraocular area punctation dense $(\mathrm{i} \leq \mathrm{pd})$. Upper paraocular area and frons reticulate-punctate $(\mathrm{i}<0.5 \mathrm{pd})$. Ocellocular area densely punctate $(\mathrm{i} \leq \mathrm{pd})$. Gena and postgena finely carinulate. Mesoscutum tessellate-imbricate, punctation moderately coarse, dense on lateral and posterior portions $(i=1-1.5 \mathrm{pd})$, sparser anteriorly and medially $(i=1-3 \mathrm{pd})$; mesoscutellum similar, with polished submedial impunctate area. Metanotum imbricate. Preëpisternum rugulose. Hypoepimeral area finely, densely punctate $(i<0.5 \mathrm{pd})$. Mesepisternum below scrobe densely punctate $(\mathrm{i} \leq \mathrm{pd})$, interspaces imbricate. Metepisternum dorsal $1 / 3$ rugoso-carinulate, ventral portion imbricate. Metapostnotum with longitudinal carinulae reaching $2 / 3-3 / 4$ distance to posterior margin, carinulae on lateral portions extending to dorsolateral slope, raised posterior margin smooth. Propodeum posterior surfaces polished tessellate, distinct punctures on lateral surface $(i=3-5 \mathrm{pd})$, irregular carinulae on posterior surface. Metasomal terga polished, finely coriarious on T1 anterior surface and apical impressed areas; punctation very fine throughout, including apical impressed areas $(i=1-2 \mathrm{pd})$. Metasomal sterna coriarious and finely, sparsely punctate $(i=2-4 \mathrm{pd})$.

Structure. Head slightly wider than long (length/width ratio=0.91-0.95). Eyes weakly convergent below. Clypeus $1 / 2$ below suborbital tangent. Gena narrower than eye. Hypostomal carinae subparallel. Pronotal dorsolateral angle obtuse. Pronotal ridge rounded, interrupted by sulcus. Tegula ovoid.
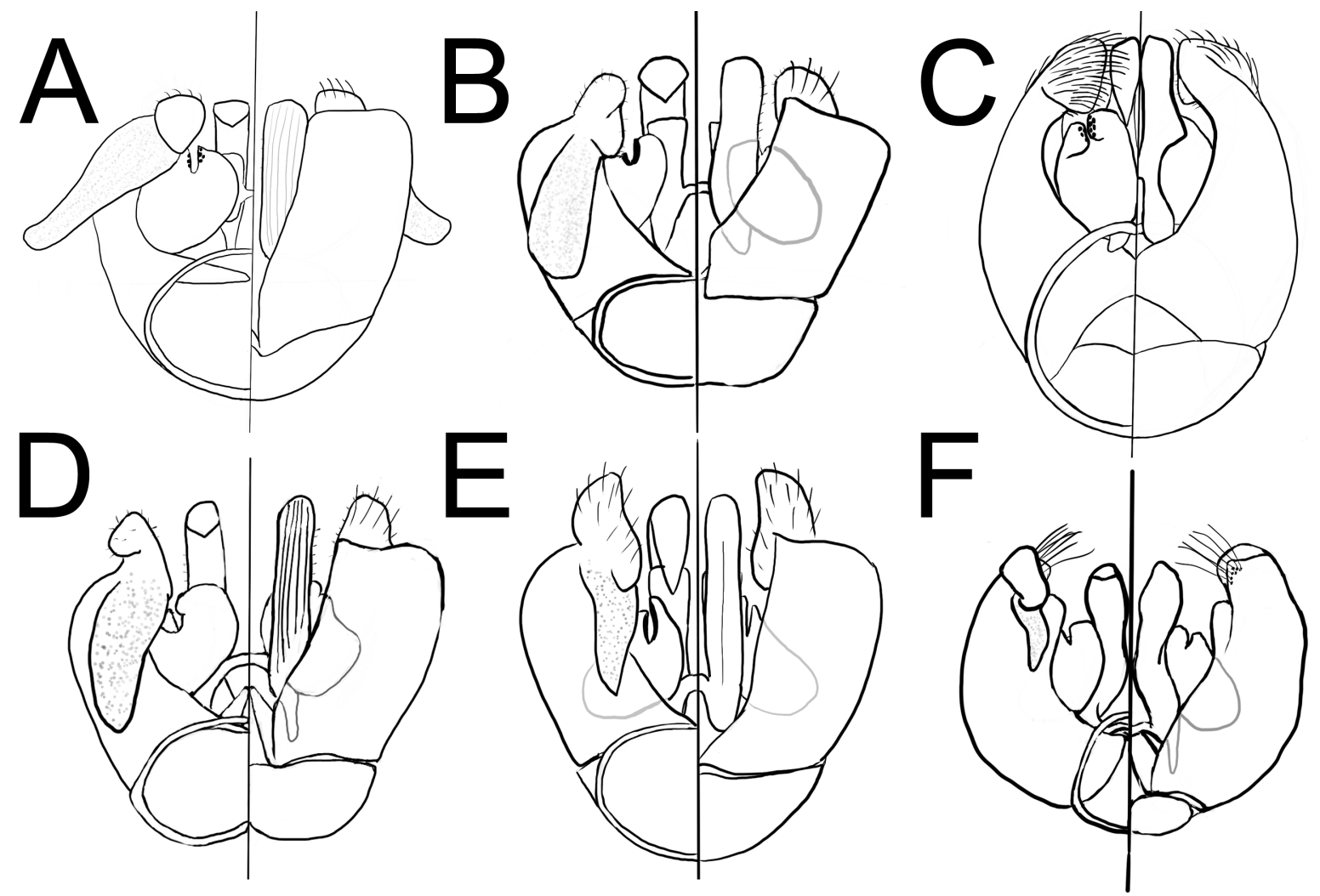

Fig. 4. Line drawings of male genital capsules in ventral (left side) and dorsal (right side) views. A. L. amona sp. nov. B. L. dispersum sp. nov. C. L. eickwortellum (Engel, 2001). D. L. enatum sp. nov. E. L. genaroi sp. nov. F. L. gundlachii (Baker, 1906). 
Submarginal cells three (1rs-m present). Distal hamuli arranged 2-1-2. Inner metatibial spur pectinate, with 3-4 branches, proximal branch much longer than width of rachis. Metapostnotum slight depressed, narrowly rounded onto posterior propodeal surface. Propodeum with lateral carina reaching dorsal margin; oblique carina distinct, delimiting ventral-posterior margin on posterior and lateral margins. T2-T4 impressed areas medially nearly equal to longitudinal length of basal area.

\section{Male}

Measurements. Head length: $1.26-1.38 \mathrm{~mm}(\overline{\mathrm{x}}=1.32 \mathrm{~mm}, \mathrm{n}=2)$; head width: $1.29-1.47 \mathrm{~mm}$ $(\overline{\mathrm{x}}=1.38 \mathrm{~mm}, \mathrm{n}=2)$; intertegular distance: $1.00-1.08 \mathrm{~mm}(\overline{\mathrm{x}}=1.04 \mathrm{~mm}, \mathrm{n}=2)$.

Similar to female except for typical sex associated characters and as follows.

Colouration. Head and mesosoma dull metallic blue. Ventral surface of F1-F11 reddish brown. Tarsi brown to brownish yellow. Metasomal terga dark brown.

Pubescence. Face below eye emargination with subappressed tomentum, partially obscuring surface. Metasomal terga without tomentum. Metasomal sterna with sparse, posteriorly directed setae.

SURFACE SCULPTURE. Mesoscutum smoother, punctation slightly sparser. Mesepisternum smooth, shining; punctures deep and distinct. Propodeum lateral surface densely punctate $(\mathrm{i}=1-1.5 \mathrm{pd})$. Metasomal terga with apical impressed areas impunctate.

Structure. Head wider than long (length/width ratio=0.94-0.97). Eyes convergent below. Ratio of pedicel, F1 and F2=11:14:25; F2-F11 more than $1.5 \times$ as long as wide. Metasomal terga with apical impressed areas medially less than $1 / 2$ length of basal area.

Terminalia. As illustrated in Fig. 4B.

\section{Distribution}

Puerto Rico (Fig. 5), British and U. S. Virgin Islands.

\section{Biology}

Lasioglossum dispersum sp. nov. has been collected from flowers of Waltheria indica L. (Malvaceae). Individuals from Guana Island were collected from Antigonon leptopus Hook. \& Arn. (Polygonaceae), Jacquemontia pentanthos (Jacq.) G.Don (Convolvulaceae) and Capparis cynophallophora L. (Capparaceae). Snelling (1993) also recorded this species from Schaefferia frutescens Jacq. as Lasioglossum sp. 2.

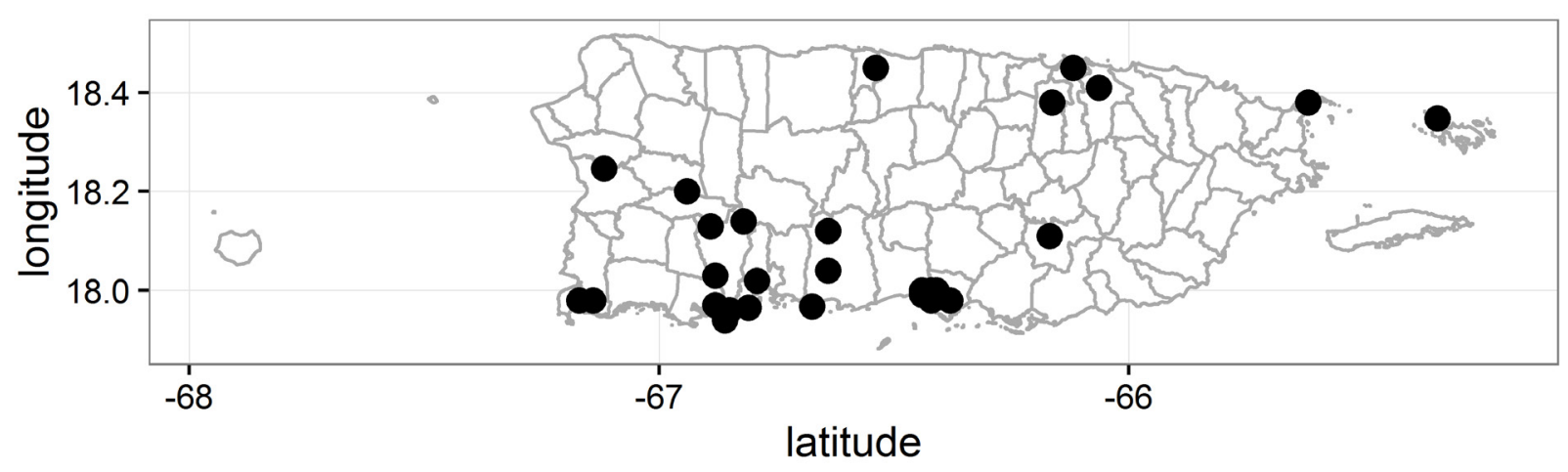

Fig. 5. Distribution map for Lasioglossum dispersum sp. nov. in Puerto Rico. 


\section{DNA barcodes}

Six specimens were successfully sequenced (BOLD Process IDs: NTCOL161-15, BOWGF3200-14, BOWGF3198-14, BOWGF3196-14, BOWGF3197-14, and BOWGF3199-14). Three specimens from the Virgin Islands cluster separately from the three individuals from Puerto Rico, suggesting that there may be limited gene flow between the islands.

\section{Remarks}

This species appears to be distributed across Puerto Rico and the Virgin Islands. There is some variation present. Several specimens from Cabo Rojo, Pedernales Province, the Dominican Republic, collected by G.C. Eickwort in 1985 and deposited at CUIC, show close affinity to L. dispersum sp. nov.

Lasioglossum (Dialictus) amona sp. nov. urn:1sid:zoobank.org:act:41557A0B-39BC-48C7-97B6-9B1091D62A18

Figs 4A, 6-8

\section{Diagnosis}

Both sexes of L. amona sp. nov. can be recognized by the combination of tegula honey-coloured and oval without extended posterior angle, mesepisternum distinctly punctate, interspaces polished and metasoma brown. The female has a distinct oblique propodeal carina, $\mathrm{T} 2-\mathrm{T} 3$ basolaterally with appressed tomentum and T2-T3 apical impressed areas distinctly, but finely punctate. In size and sculpturing, L. amona sp. nov. is similar to members of the L. parvum species complex, but can be recognized by the unmodified tegula. It is closely allied to $L$. dispersum sp. nov., but differs in the female by the smoother mesepisternal sculpturing of interspaces, finer mesoscutal punctation, smoother dorsolateral slope and less distinct oblique carina. The male can only be distinguished from those of $L$. dispersum sp. nov. by the paler green colour and honey-coloured tegula. Lasioglossum genaroi sp. nov. is related, but has rugose to reticulate-rugose mesepisternal sculpturing and typically a larger body size, which in females is significantly larger on average, and a darker blue colour.

\section{Etymology}

The specific epithet is a noun in apposition taken from the Taíno name for Mona Island.

\section{Material examined}

\section{Holotype}

PUERTO RICO: + , Mona Island, 5 Apr. 1944, J.A. Ramos leg. (NMNH). [Original label: Mona Island, 5 - Apr. 1944 // J.A. Ramos collector // HOLOTYPE Lasioglossum (Dialictus) amona.]

\section{Paratypes}

PUERTO RICO: Mona Island: 1 + , topotypical (JBWM); 1 क, 30 May 1963, A. Berrios leg. (JBWM); 1 q, Mona Island, 19-26 Jan. 2001, M.A. Garcia and J. Sustache leg. (AMNH); 2 우, 4 ठ $\delta^{\lambda}$, Ulvero, road above, 11 Nov. 1992, Snelling and Torres leg. (LACM); 2 우, 1 ${ }^{\lambda}$, Mona Island, Malaise trap, 28-30 Oct. 1991, R.R. Snelling and J.A. Torres leg. (LACM).

\section{Description}

Female

Measurements. Head length: $1.26-1.34 \mathrm{~mm}(\overline{\mathrm{x}}=1.29 \mathrm{~mm}, \mathrm{n}=8)$; head width: $1.33-1.47 \mathrm{~mm}$ $(\overline{\mathrm{x}}=1.40 \mathrm{~mm}, \mathrm{n}=8)$; intertegular distance: $0.96-1.08 \mathrm{~mm}(\overline{\mathrm{x}}=1.01 \mathrm{~mm}, \mathrm{n}=8)$.

Colouration. Head and mesosoma dull metallic golden green, except as follows. Labrum reddish brown. Mandible brown with red apex. Clypeus distal half dark brown. Antenna dark brown, F2-F10 
with ventral surface dark reddish brown. Tegula honey-coloured. Wing membrane hyaline with dark setae, venation and pterostigma honey-coloured. Legs dark brown, except medio- and distitarsi reddish brown, basitarsi infused with reddish bown. Metasomal terga brown, apical areas brownish yellow.

PubesCENCE. Dull white. Relatively sparse erect setae throughout, without tomentum, except narrow basolateral patches of T2-T3 and basally on T4. T1 with complete fan of appressed setae on anterior surface. T2 without apical fimbriae, T3-T4 with only sparse fine setae on apical impressed areas. Scopa well developed on hind leg and metasomal sterna.

SuRfaCE SCULPTURE. Face imbricate, punctation moderately coarse, finer on frons. Clypeus punctation sparse $(i=1-2.5 \mathrm{pd})$, surface smooth distally $(\mathrm{i}=2-3 \mathrm{pd}$ ), supraclypeal area with punctures moderately
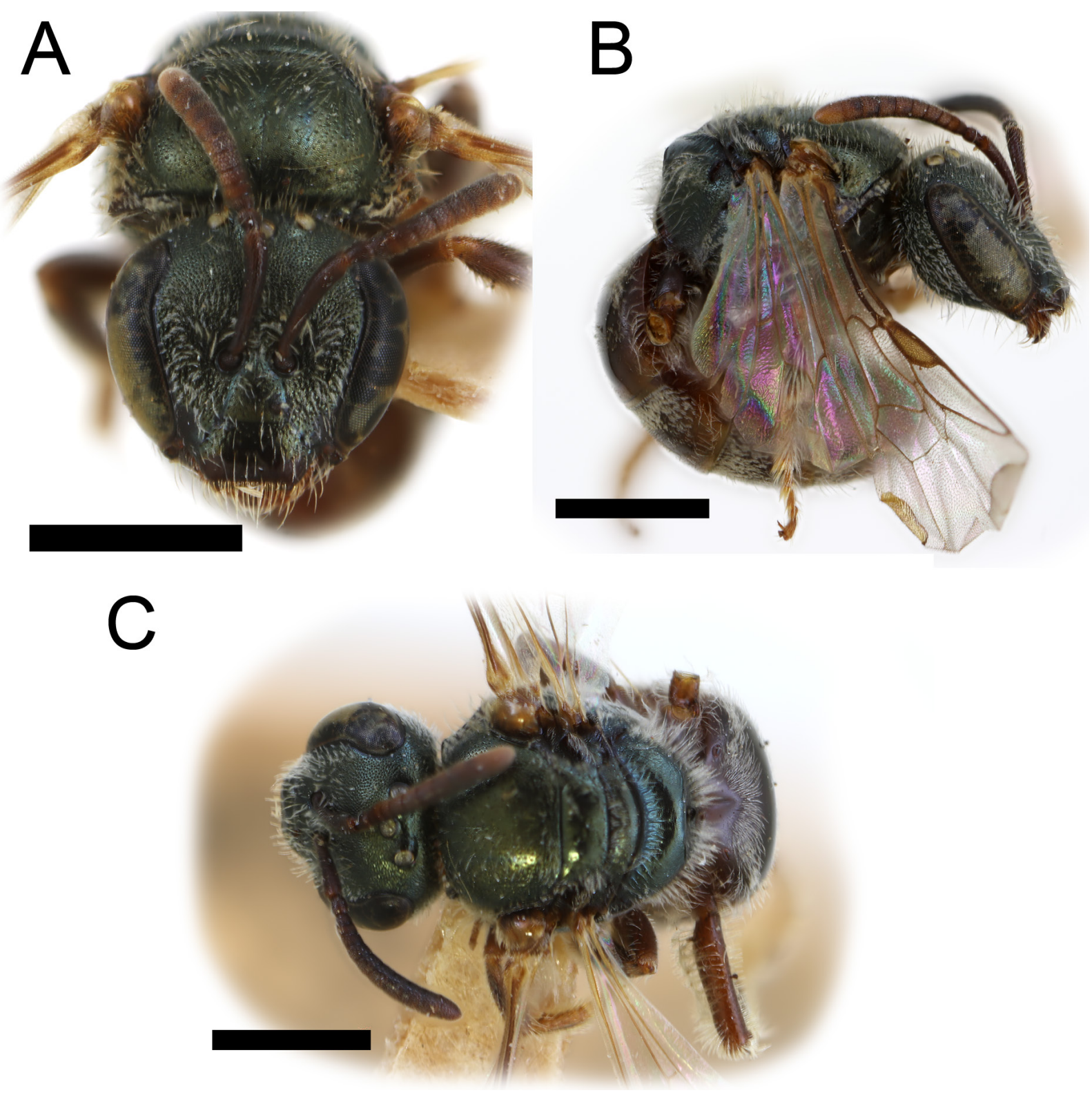

Fig. 6. Lasioglossum amona sp. nov., +. A. Face. B. Lateral habitus. C. Dorsal habitus. Scale bars $=1 \mathrm{~mm}$. 
dense $(\mathrm{i}=1-1.5 \mathrm{pd})$ and lower paraocular area punctation dense $(\mathrm{i} \leq \mathrm{pd})$. Upper paraocular area and frons reticulate-punctate $(\mathrm{i}<0.5 \mathrm{pd})$. Ocellocular area densely punctate $(\mathrm{i} \leq \mathrm{pd})$. Gena and postgena finely carinulate. Mesoscutum imbricate, punctation dense on lateral and posterior portions $(i=1-1.5$ pd), sparser anteriorly and medially $(i=1-3 \mathrm{pd})$; mesoscutellum similar, with polished submedial impunctate area. Metanotum imbricate-punctate. Preëpisternum rugulose-reticulate. Hypoepimeral area finely, densely punctate $(i<0.5 \mathrm{pd})$. Mesepisternum below scrobe densely punctate $(\mathrm{i} \leq \mathrm{pd})$, interspaces weakly imbricate. Metepisternum dorsal $1 / 3$ rugoso-carinulate, ventral portion imbricate. Metapostnotum with longitudinal carinulae reaching $2 / 3-3 / 4$ distance to posterior margin, carinulae on lateral portions extending to dorsolateral slope, raised posterior margin smooth. Propodeum posterior surfaces polished tessellate, distinct punctures on lateral surface $(i=3-5 \mathrm{pd})$. Metasomal terga polished, finely coriarious on T1 anterior surface and apical impressed areas; punctation very fine throughout, including apical impressed areas ( $\mathrm{i}=1-2 \mathrm{pd})$. Metasomal sterna coriarious and finely, sparsely punctate $(\mathrm{i}=2-4 \mathrm{pd})$.
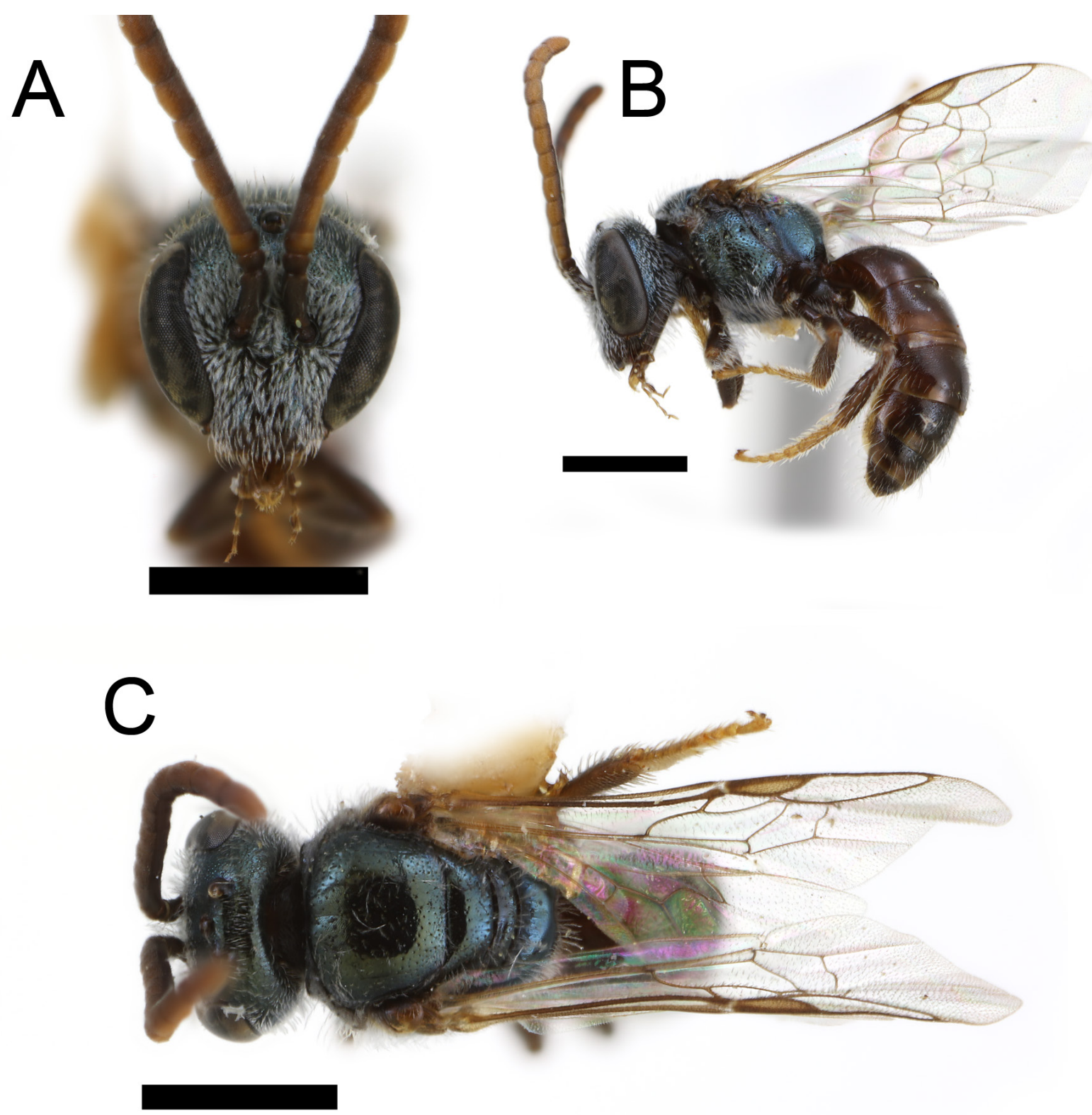

Fig. 7. Lasioglossum amona sp. nov., §̂. A. Face. B. Lateral habitus. C. Dorsal habitus. Scale bars $=1 \mathrm{~mm}$. 
STRUCTURE. Head slightly wider than long (length/width ratio=0.91-0.94). Eyes weakly convergent below. Clypeus $2 / 3$ below suborbital tangent. Gena narrower than eye. Hypostomal carinae subparallel. Pronotal dorsolateral angle obtuse. Pronotal ridge rounded, interrupted by sulcus. Tegula ovoid. Submarginal cells three (1rs-m present). Distal hamuli arranged 2-1-2. Inner metatibial spur pectinate, with 3-4 branches, proximal branch much longer than width of rachis. Metapostnotum slight depressed, narrowly rounded onto posterior propodeal surface. Propodeum with lateral carina reaching dorsal margin; oblique carina present, delimiting ventral-posterior margin on posterior and lateral margins. T2-T4 impressed areas medially nearly equal to longitudinal length of basal area.

\section{Male}

MeAsurements. Head length: $1.23-1.40 \mathrm{~mm}(\overline{\mathrm{x}}=1.33 \mathrm{~mm}, \mathrm{n}=5)$; head width: $1.28-1.48 \mathrm{~mm}$ $(\overline{\mathrm{x}}=1.39 \mathrm{~mm}, \mathrm{n}=5)$; intertegular distance: $0.91-1.05 \mathrm{~mm}(\overline{\mathrm{x}}=1.00 \mathrm{~mm}, \mathrm{n}=5)$.

Similar to female except for typical sex associated characters and as follows.

Colouration. Head and mesosoma dull metallic bluish green. Ventral surface of F1-F11 brownish yellow. Tarsi brown to brownish yellow. Metasomal terga dark brown, with reddish brown apical margins.

Pubescence. Face below eye emargination with subappressed tomentum, partially obscuring surface. Metasomal terga without tomentum. Metasomal sterna with sparse, posteriorly directed setae.

SURFACE SCULPTURE. Mesoscutum smoother, punctation slightly sparser. Mesepisternum smooth, shining; punctures deep and distinct. Propodeum lateral surface densely punctate $(\mathrm{i}=1-1.5 \mathrm{pd})$. Metasomal terga with apical impressed areas impunctate.

Structure. Head wider than long (length/width ratio=0.95-0.96). Eyes convergent below. Ratio of pedicel, F1 and F2=10:12:26; F2-F11 more than 1.5× as long as wide. Metasomal terga with apical impressed areas medially less than $1 / 2$ length of basal area.

Terminalia. As illustrated in Fig. 4A.

\section{Distribution}

This species is apparently endemic to Mona Island (Fig. 8).

\section{Remarks}

Lasioglossum amona sp. nov. is very closely related to $L$. dispersum sp. nov. The differences in microsculpture and structure are suggestive of species-level differences, but it seems probable that these two species share a recent ancestor. Additional study is needed to determine whether the morphological and geographical basis for recognizing L. amona sp. nov. as distinct from L. dispersum sp. nov. is valid.

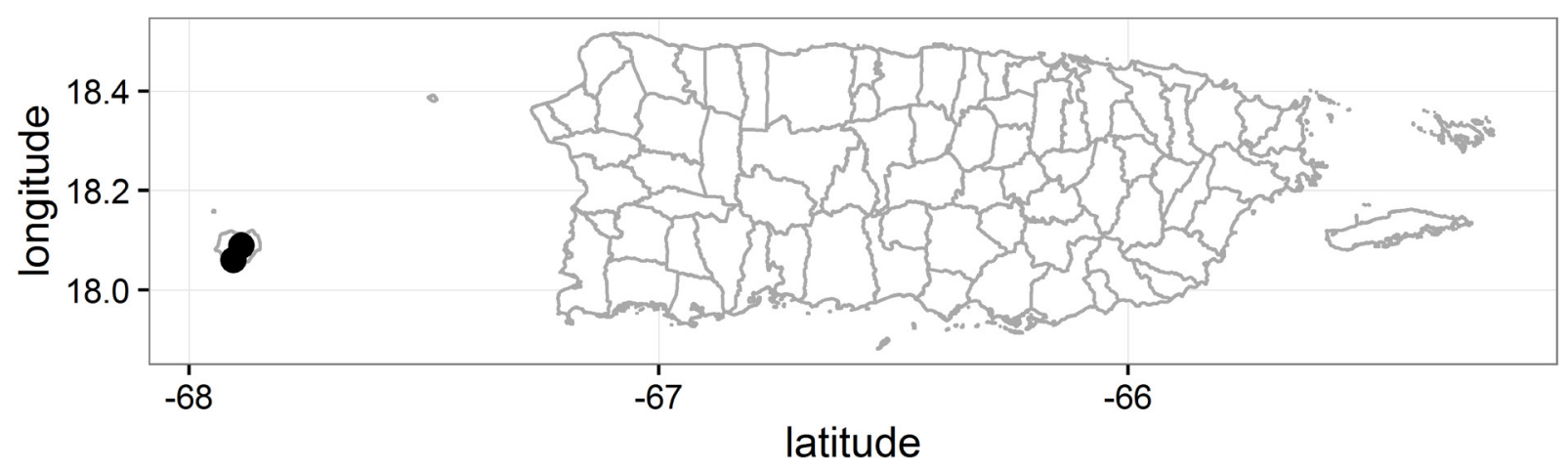

Fig. 8. Distribution map for Lasioglossum amona sp. nov. 


\section{Lasioglossum (Dialictus) enatum sp. nov. urn:1sid:zoobank.org:act:2D162288-635C-4D9C-9887-F1453F3F9EAE \\ Figs 4D, 9-11}

\section{Diagnosis}

Both sexes of Lasioglossum enatum sp. nov. can be recognized by tegula punctate, extended posteriorly to form small angle, mesepisternum punctate and metasoma brown. The shape of the tegula distinguishes it from all other species of $L$. (Dialictus) from Puerto Rico, except L. monense sp. nov. on Mona Island. The female of $L$. monense sp. nov. has a pale metasoma and obscure punctation on the supraclypeal area. The male of L. enatum sp. nov. has the mesoscutum and mesepisternum more polished and shiny than in males of L. monense sp. nov. Other members of the L. parvum species complex, to which it belongs, share this character. In the female, the complete absence of punctures on the apical impressed margins distinguishes L. enatum sp. nov. from L. parvum and L. busckiellum, which both have minute setose punctures in this area. In the male, the appressed sternal setae and dark tarsi distinguish L. enatum sp. nov. from $L$. parvum, which has sternal setae erect and tarsi pale.

\section{Etymology}

The specific epithet enatum is a participle of the Latin enascor, meaning having sprouted or sprung forth.

\section{Material examined}

\section{Holotype}

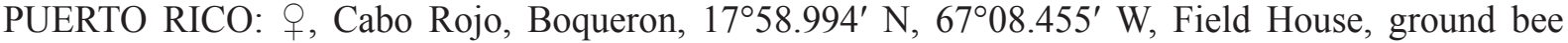
bowl, 12-16 May 2014, S.G. Prado leg. (JBWM). [Original label: USA, PR Boqueron N1758.994' W67 $08.455^{\prime}$, Field House, ground bee bowl, 12-16 May 2014, S.G. Prado, \#49 // HOLOTYPE Lasioglossum (Dialictus) enatum Gibbs.]

\section{Paratypes}

PUERTO RICO: Aguadilla: 1 đ, [no locality], Jan. 1899, A. Busck leg. (NMNH); 1 , Playa Bajuras, Jul. 1999, J.A. Genaro leg. (JAGC). - Añasco: 2 우, [no locality], 3 Jul. 1917, H. Morrison leg. (NMNH). - Arecibo: 1 ㅇ, [no locality], ex Gundlachia corymbosa, 4 Oct. 1922, G.N. Wolcott leg. (NMNH); 1 ㅇ, [no locality], flowers in field, 19 Dec. 1933, Anderson and Mills leg. (NMNH). - Arroyo:

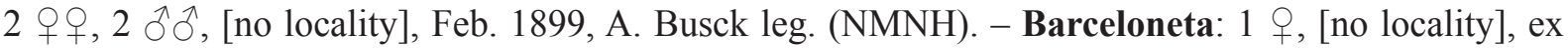
Barbieria pinnata, 21 Feb. 1933, Anderson, Faxon and Mills leg. (NMNH); 1 ㅇ, [no locality], ex rose flower, 15 Nov. 1932, Anderson, Mills and Faxon leg. (NMNH). - Bayamón: 1 đ̂, Stanwood Grove, 26 Jun. 1917, H. Morrison leg. (NMNH). - Cabo Rojo: 1 ㅇ, [no locality], Dec. 1960, R. Cotte leg.

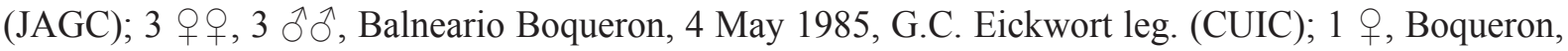
Cabo Rojo National Wildlife Refuge, USFWS, $17^{\circ} 58.816^{\prime} \mathrm{N}, 67^{\circ} 10.231^{\prime} \mathrm{W}$, ground bee bowl, 27 May13 Jun. 2014, S.G. Prado leg. (NCSU); 1 đo, same locality as preceding, net, 3 Jun. 2014, S.G. Prado

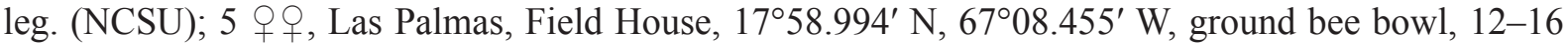
May 2014, S.G. Prado leg. (JBWM); 1 q, same collection data as preceding, 6 Jun. 2014 (NCSU);

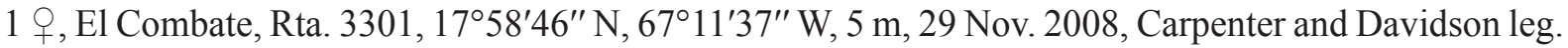
(AMNH). - Cayey: 1 , , Bo. Beatriz, 5 Nov. 1992, Snelling and Torres leg. (LACM). - Cidra: 2 우,

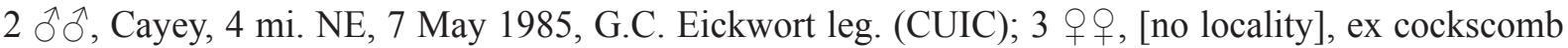
flower, 5 Aug. 1932, Anderson, Faxon and Mills leg. (NMNH); 1 + , [no locality], ex asparagus flower, 5 Aug. 1932, Anderson, Faxon and Mills leg. (NMNH). - Corozal: 1 + , [no locality], 1 Jan. 1920, G.N.

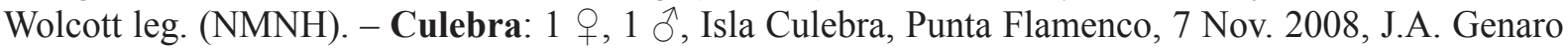

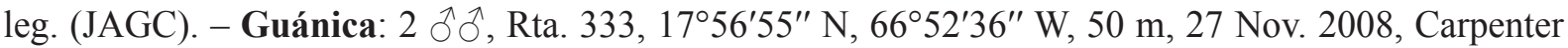
and Davidson leg. (AMNH); 1 ๆ, Guánica, Oct. 1997, J.A. Genaro leg. (JAGC); 1 ô, Insular Forest, 


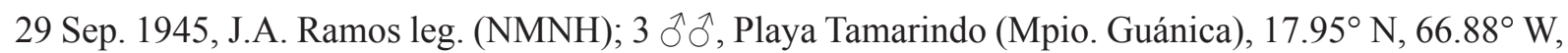
$10 \mathrm{ft}$ a.s.1., ex Heliotropium curassavicum, 26 Sep. 1998, R.R. Snelling leg. (LACM); 1 §̊, same collection

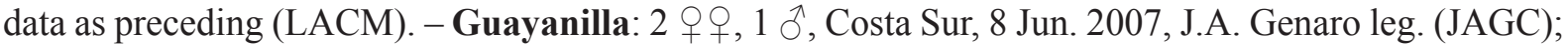
6 웅, 1 ㄱ, 8 Jun. 2007, J.A. Genaro leg. (JAGC); 2 우, Playa Ventana, Oct. 2008, J.A. Genaro leg. (JAGC); 1 q, Punta Verraco, 14 Nov. 2010, J.A. Genaro leg. (JAGC). - Humacao: 4 우, 4 o, Playa de Humacao, 11 May 1985, G.C. Eickwort leg. (CUIC). - Isabela: 1 + , [no locality], ex Coriandrum sativum, 7 Apr. 1948, L.F. Martorell leg. (NMNH); 1 , [no locality], ex Borreria verticillata, 29 Jun. 1978, R.I. Sailer leg. (FSCA); 1 §ै, [no locality], Nov. 1981, N. Semiday leg. (FSCA); 1 ㅇ, [no locality], 2-3 Feb. 1940, R.A. Maldonado leg. (JAGC); 1 + , [no locality], 24 Jan. 1971, L.J. Joly T. leg. (JAGC); 2 우, Punta Rosario, nr. Isabela, 14 Jan. 1963, P.J. Spangler leg. (NMNH). - Lajas: 1 , , Cartagena Lagoon, Feb. 1935, [illegible] leg. (NMNH); 1 q, Laguna Cartagena, 1 mi. S of Llanos, 4 May 1985, G.C. Eickwort leg. (CUIC); 8 우, 1 §స, Isla Maguey, Parguera, 19 Dec. 1962, P. Spangler leg. (NMNH); 2 우, 1 §̂, same collection data as preceding, 20 Dec. 1962 (NMNH). - Loíza: 1 ㅇ, B. [osque] Piñones, \#74, 5 Nov. 1985, J. Torres leg. (LACM); 4 우, Isl. Juan Pérez, \#106, 28 Jun. 1986, J. Torres leg.

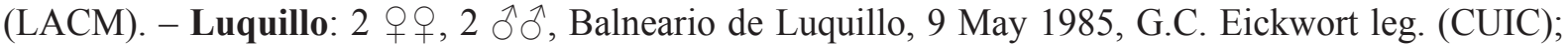

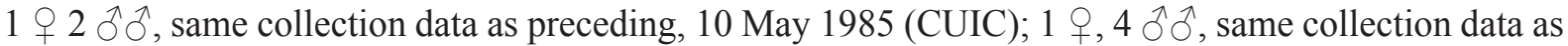
preceding, 13 May 1985 (CUIC); 2 우, 1 , same collection data as preceding, 14 May 1985 (CUIC).

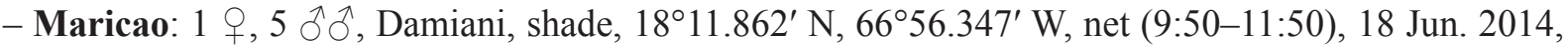

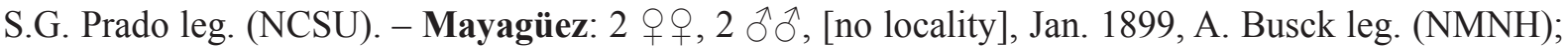

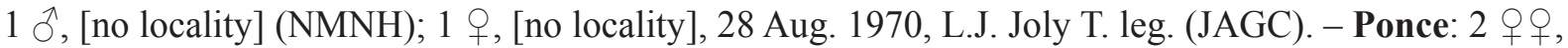
1 ऽ, Cayo Ratones, May 1999, J.A. Genaro leg. (JAGC); 1 + , La Rita, near Ponce, Oct. 2010, J.A. Genaro leg. (JAGC); 1 , same collection data as preceding, 14 May 2008 (JAGC); $1 \hat{\jmath}$, same collection data as preceding, May 2008 (JAGC); 1 q, Playa El Tuque, 5 mi. W of Ponce, 4 May 1985, G.C. Eickwort leg. (CUIC); 1 + , same collection data as preceding, 5 May 1985 (CUIC); 1 \&, Playa Guánica, Jul. 1999, J.A. Genaro leg. (JAGC); 2 $q$, Ponce, 7 May 1985, G.C. Eickwort leg. (CUIC); 9 우, Vasquez, sun, $18^{\circ} 07.4616^{\prime} \mathrm{N}, 66^{\circ} 38.2638^{\prime} \mathrm{W}$, Malaise trap, 17 Jun.-15 Jul. 2014, S.G. Prado leg. (NCSU); 9 우, same collection data as preceding, 15 Jul.-12 Aug. 2014 (NCSU); 5 우, 2 § ${ }^{\text {, }}$, same collection data as preceding, 12 Aug. -9 Sep. 2014 (NCSU); 5 우, 1 §, same collection data as preceding, 9 Sep. -7 Oct. 2014 (NCSU); 1 +, same locality as preceding, ground bee bowl, 17 Jun.-15 Jul. 2014, S.G. Prado leg. (NCSU). - Sabana Grande: $2 \widehat{\partial} \partial^{\lambda}$, Susua Forest Reserve, 3 mi. NE of Sabana Grande, 3 May 1985,

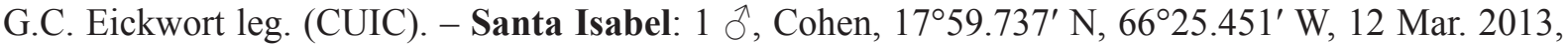

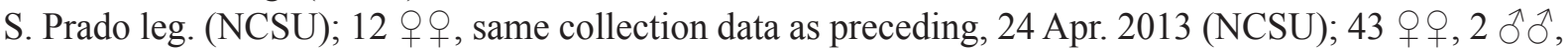

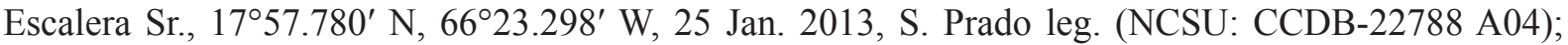
5 우, same collection data as preceding, $30 \mathrm{Jan} .2013$ (NCSU); 7 우, $2 \hat{\jmath} \widehat{\jmath}$, same collection data as

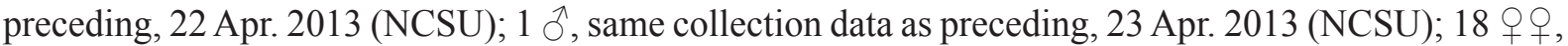

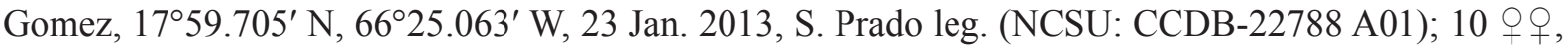
$1 \hat{\jmath}$, same collection data as preceding, 13 Mar. 2013 (NCSU); 3 우, same collection data as preceding, 2 May 2013 (NCSU); 1 ㅇ, NW River, $18^{\circ} 0.017^{\prime}$ N, 66 $26.115^{\prime}$ W, 26 Jan. 2013, S. Prado leg. (NCSU);

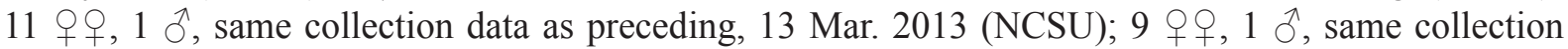

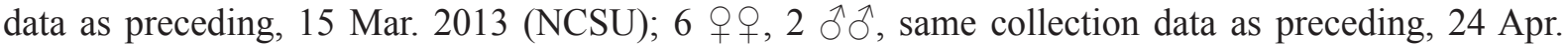
2013 (NCSU: CCDB-22788 A03, CCDB-22788 A10); 7 우, 3 $\hat{\delta} \hat{0}$, same collection data as preceding,

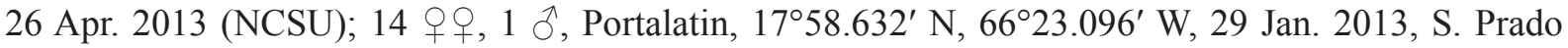
leg. [NCSU: CCDB-22788 A02]; 4 우, same collection data as preceding, 12 Mar. 2013 (NCSU);

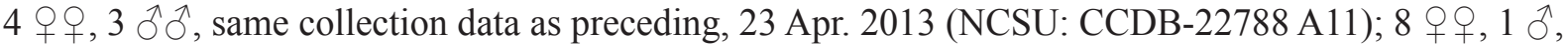
Roriguez, $18^{\circ} 00.017^{\prime} \mathrm{N}, 66^{\circ} 26.863^{\prime} \mathrm{W}, 15$ Mar. 2013, S. Prado leg. (NCSU); 9 우 , Roriguez, same

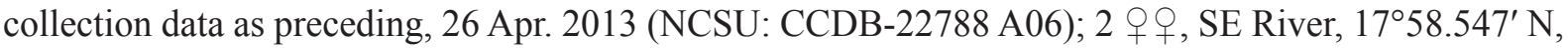
$66^{\circ} 25.063^{\prime} \mathrm{W}, 22$ Jan. 2013, S. Prado leg. (NCSU); 5 우, $2 \widehat{\delta}^{\widehat{\jmath}}$, same collection data as preceding, 28 Jan. 2013 (NCSU); 1 t, same collection data as preceding, 13 Mar. 2013 (NCSU); 3 우, 1 ○, same collection data as preceding, 15 Mar. 2013 (NCSU); 4 q $q$, same collection data as preceding, 26 Apr. 2013 (NCSU); 2 우, 1 §ે, same collection data as preceding, 2 May 2013 (NCSU); 2 우, 
SW River, $17^{\circ} 59.496^{\prime}$ N, 66 $26.582^{\prime}$ W, 22 Jan. 2013, S. Prado leg. (NCSU); 1 9 , same collection data as preceding, 13 Mar. 2013 (NCSU); 5 우, same collection data as preceding, 15 Mar. 2013 (NCSU); 19 웅, $3 \widehat{\partial} \widehat{\jmath}$, same collection data as preceding, 24 Apr. 2013 (NCSU); 8 q $ᄋ, 1$ ô, same collection data as preceding, 26 Apr. 2013 (NCSU: CCDB-22788 A05); 1 \%, Santa Isabel, 4 mi. N, 7 May 1985, G.C. Eickwort leg. (CUIC). - Quebradilla: 2 우, 1 §ิ, El Tunel, Balneiro, Guajataca, 30 May 2006, J.A. Genaro (JAGC). - Yauco: 6 우, A7 Church site, $18^{\circ} 01.830^{\prime} \mathrm{N}, 66^{\circ} 53.042^{\prime} \mathrm{W}$, ground bee bowl, 23 May-20 Jun. 2014, S.G. Prado leg. (NCSU); 1 q, same collection data as preceding, 20 Jun.-18 Jul.

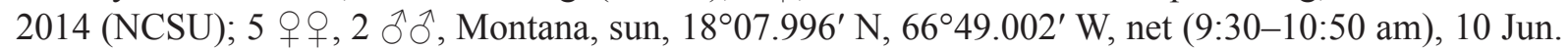
2014, S.G. Prado leg. (NCSU); 1 ㅇ, Villa Cecilia, $18^{\circ} 08.371^{\prime}$ N, 66 $69.230^{\prime}$ W, net (9:15-10:45 am), 4 Jun. 2014, S.G. Prado leg. (NCSU).

US VIRGIN ISLANDS: St. Croix: 2 + + , Southcentral (?), Exp. Sta. Grounds, 13 Jun. 1917, H. Morrison

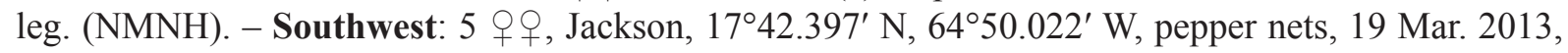
S. Prado leg. (NCSU); 1 , same locality as preceding, pumpkin bowls, 19 Mar. 2013, S. Prado leg.

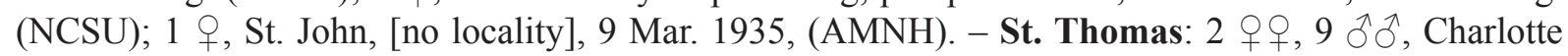
Amalie, 31 May 1917, H. Morrison leg. (NMNH). - Northside: 1 , , 1 ઈ, Swept Magen Bay, 3 Jun. 1917, H. Morrison leg. (NMNH).

\section{Other material}

BRITISH VIRGIN ISLANDS: Guana Island: 1 , The Flat, Malaise trap, 2-3 Oct. 2007, B.D. Valentine family leg. (AMNH); 1 , same collection data as preceding, 20 Oct. 2007 (AMNH); 1 , , same locality as preceding, sweeping, 9 Oct. 2007, B.D. Valentine family leg. (AMNH); 1 , , same collection data as preceding, 10 Oct. 2007 (AMNH); 1 \%, same collection data as preceding, 22 Oct. 2006 (AMNH); 1 , , flowers, 3 Oct. 2009, D. Valentine leg. (AMNH); 2 q $q$, same collection data as preceding, 6 Oct. 2009 $(\mathrm{AMNH}) ; 1$, , same collection data as preceding, 8 Oct. 2009 (AMNH); 1 \&, same collection data as preceding, 9 Oct. 2009 (AMNH); 1 , , same collection data as preceding, 10 Oct. 2009 (AMNH); 2 우,

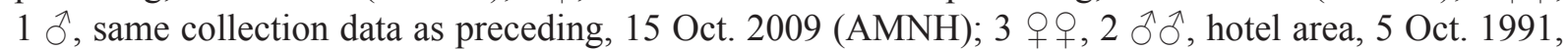
R.R. Snelling leg. (LACM); 3 $q$, , same collection data as preceding, 7 Oct. 1992 (LACM); $2 \hat{\jmath} \widehat{\partial}$, same collection data as preceding, 11 Oct. 1992 (LACM); 1 q, same collection data as preceding, 15 Oct. 1991 (LACM); $2 \widehat{\widehat{\partial}}$, same collection data as preceding, 19 Oct. 1991 (LACM); 3 q 9 , same collection data as preceding, 21 Oct. 1991 (LACM); 3 우, 2 กิ $\widehat{\text {, }}$, same collection data as preceding, 22 Oct.

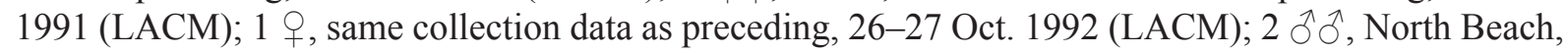
$18^{\circ} 29^{\prime}$ N, 64³4' W, 9 Jul. 1993, R.R. Snelling leg. (LACM); 2 우, 1 đo, same locality as preceding, ex Jacquemontia pentantha, 8 Oct. 1992, R.R. Snelling leg. (LACM); 1 ㅇ, 1 §े, same collection data as

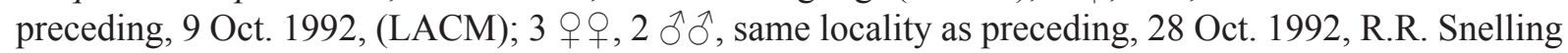
leg. (LACM); 1 , , north side, at night, 4 Oct. 2009, D.M. Dennis and W. Lu leg. (AMNH); 4 q $q, 1$ ô, plantation area, $18^{\circ} 28.7^{\prime} \mathrm{N}, 64^{\circ} 34.4^{\prime} \mathrm{W}, 27$ Jun. 1993, R.R. Snelling leg. (LACM); 1 Jै, same collection data as preceding, 28 Jun. 1993 (LACM); 2 우, 1 O, plantation area, 25 Oct. 1992, R.R. Snelling leg. (LACM); 1 , plantation area, ex Cardiospermum micranthum [sic], 31 Oct. 1992, R.R. Snelling leg. (LACM); 1 q, Quail Dove Ghut, Malaise trap, Nov. 2006, W.-P. Liao leg. (AMNH); 7 q , White Beach, $18^{\circ} 28.7^{\prime} \mathrm{N}, 64^{\circ} 34.5^{\prime} \mathrm{W}, 28$ Jun. 1993, R.R. Snelling leg. (LACM); 1 ㅇ, 1 J, same collection data as preceding, 4 Jul. 1993 (LACM); 6 q,, 2 $\hat{\jmath}$, same locality as preceding, ex Casine xylocarpum [sic], 29 Jun. 1993, R.R. Snelling leg. (LACM); 2 q $q$, same collection data as preceding, 30 Jun. 1993 (LACM); 1 + , same collection data as preceding, 2 Jul. 1993 (LACM); 1 , same collection data as

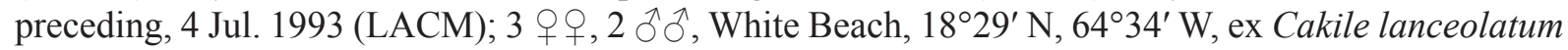
[sic], 5 Jul. 1993, R.R. Snelling leg. (LACM); 1 +, same locality as preceding, 9 Jul. 1993, R.R. Snelling leg. (LACM); 18 우우, 4 §ðð, White Beach, ex Cakile lanceolatum, 10 Oct. 1992, R.R. Snelling leg. (LACM); 2 우, same locality as preceding, Malaise trap, dry evergreen forest, 13-17 Oct. 1992, R.R.

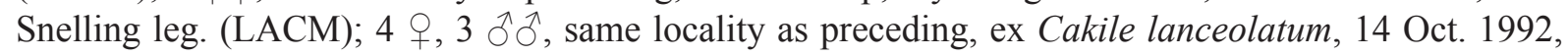
R.R. Snelling leg. (LACM); 1 ㅇ, same locality as preceding, ex Ipomoea pes-capri braziliensis [sic], 
16 Oct. 1992, R.R. Snelling leg. (LACM); 1 ㅇ, same locality as preceding, ex Coccoloba uvifera,

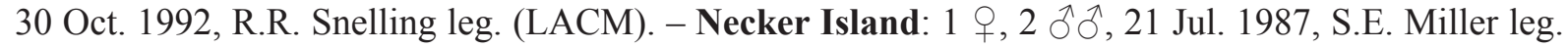

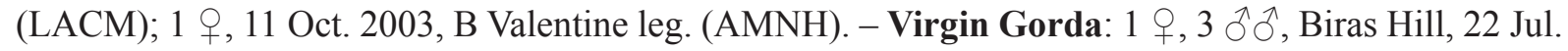
1998, S.E. Miller and C. O’Connell leg. (LACM).

\section{Description}

\section{Female}

Measurements. Head length: $1.16-1.39 \mathrm{~mm}(\overline{\mathrm{x}}=1.31 \mathrm{~mm}, \mathrm{n}=11)$; head width: $1.21-1.45 \mathrm{~mm}$ $(\overline{\mathrm{x}}=1.36 \mathrm{~mm}, \mathrm{n}=11)$; intertegular distance: $0.86-1.11 \mathrm{~mm}(\overline{\mathrm{x}}=0.98 \mathrm{~mm}, \mathrm{n}=11)$.

Colouration. Head and mesosoma dull metallic golden green with blue reflections, except as follows. Labrum brown. Mandible brown with red apex. Clypeus distal half dark brown. Supraclypeal area

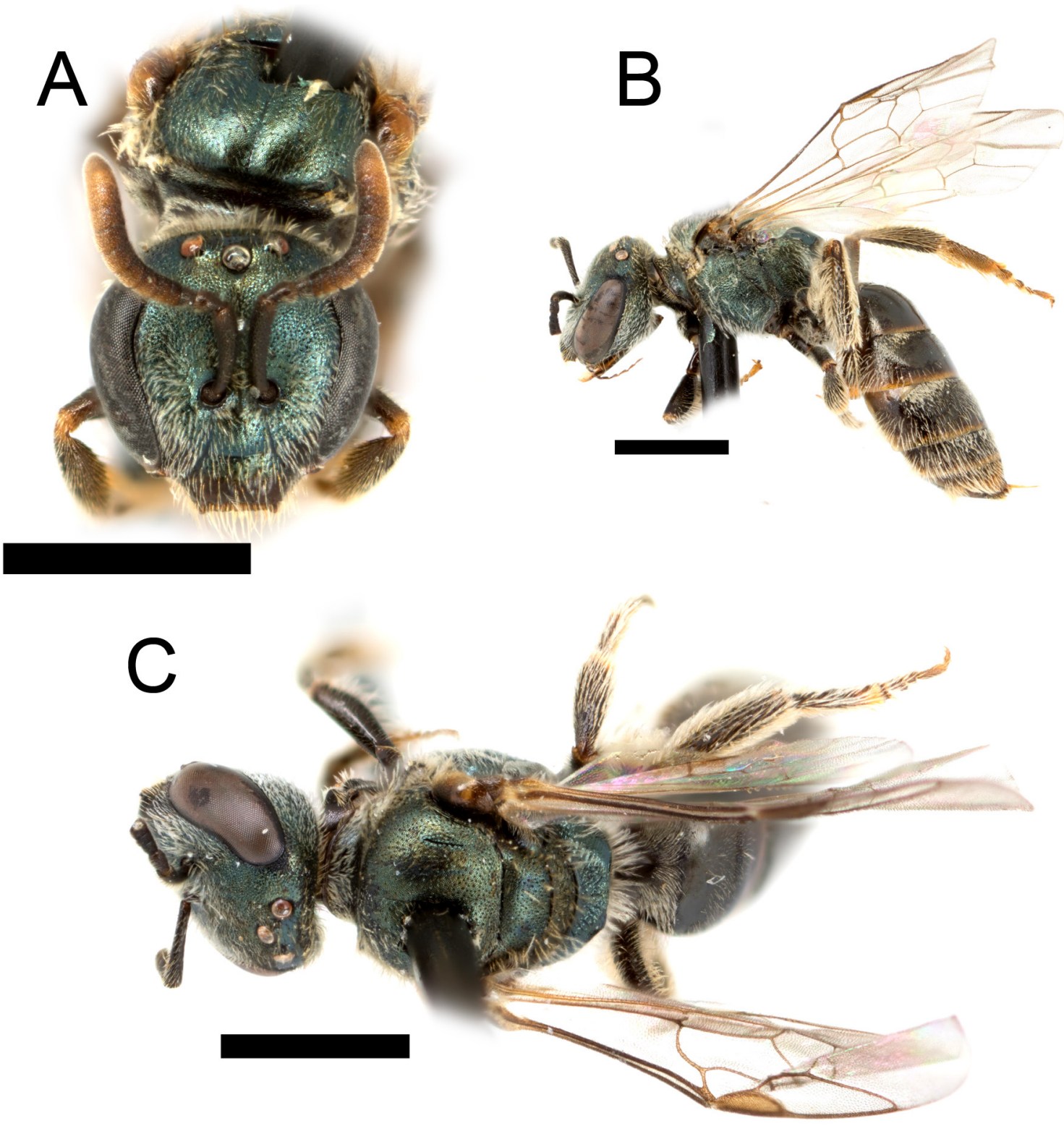

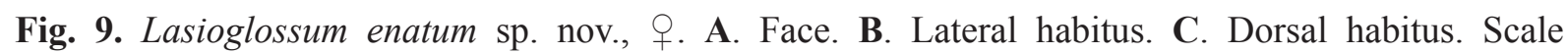
bars $=1 \mathrm{~mm}$. 
bronze. Antenna dark brown, F9-F10 with ventral surface dark reddish brown. Tegula dark reddish brown to honey-coloured. Wing membrane hyaline with dark setae, venation and pterostigma brown. Legs dark brown, except medio- and distitarsi reddish brown. Metasomal terga brown, apical impressed areas reddish brown with yellowish posterior rim.

PuBESCENCE. Dull white. Relatively sparse erect setae throughout, without tomentum, except basolateral patches of T2-T3 and basally on T4. T1 with complete fan of appressed setae on anterior surface. T2 without apical fimbriae, T3-T4 with sparse fine seta on apical impressed areas, sparse fimbriae laterally. Scopa well developed on hind leg and metasomal sterna.

SuRFACE SCULPTURE. Face imbricate, punctation moderately fine, finer on frons, coarse on distal clypeus. Clypeus punctation sparse $(i=1-2.5 \mathrm{pd})$, finer and denser proximally, surface smooth distally $(i=2-3 \mathrm{pd})$,
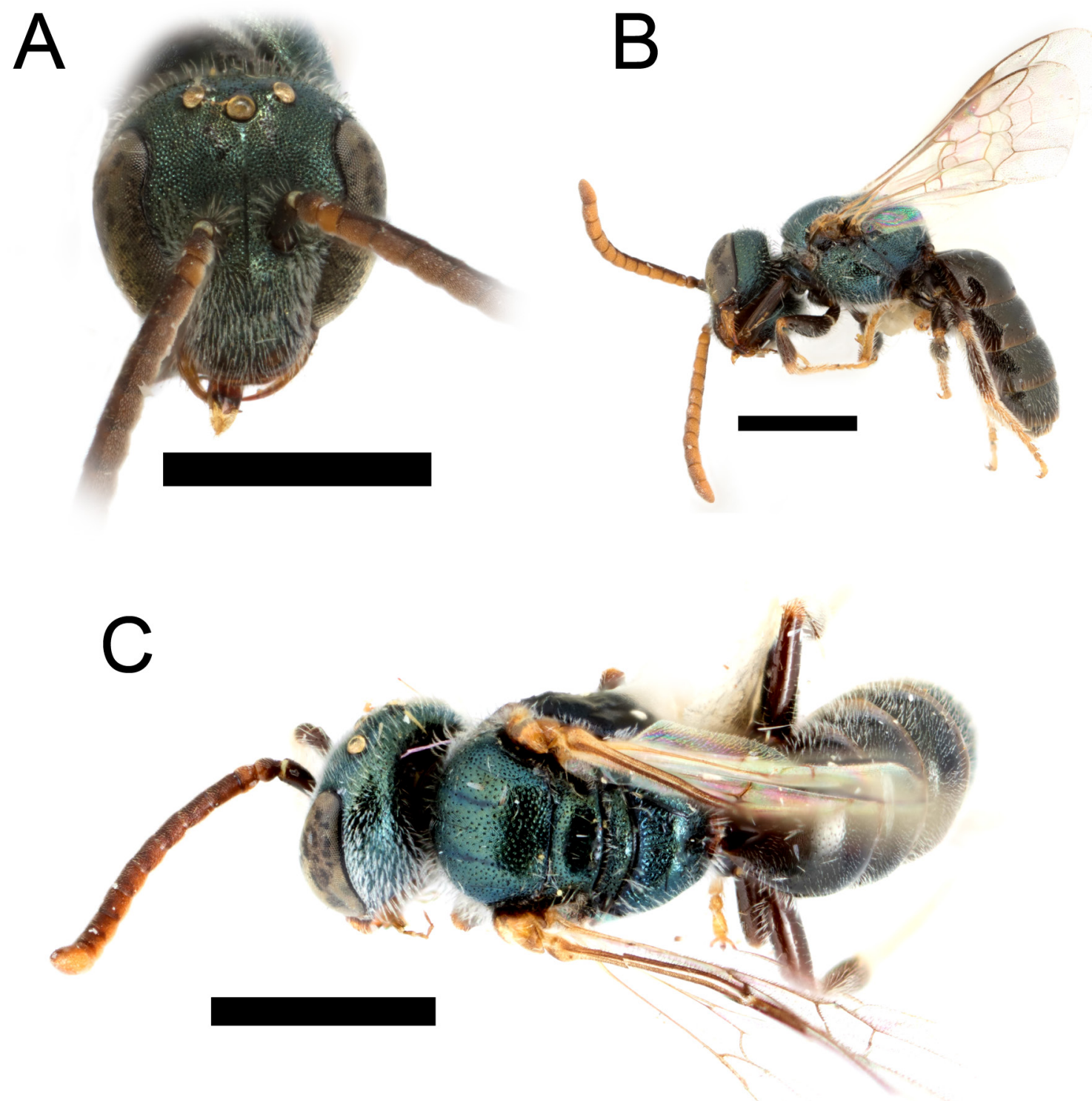

Fig. 10. Lasioglossum enatum sp. nov., Oิ. A. Face. B. Lateral habitus. C. Dorsal habitus. Scale bars $=1 \mathrm{~mm}$. 
supraclypeal area with punctures moderately dense $(\mathrm{i}=1-1.5 \mathrm{pd})$ and lower paraocular area punctation dense $(i \leq p d)$. Upper paraocular area and frons reticulate-punctate $(i<0.5 \mathrm{pd})$. Ocellocular area densely punctate $(\mathrm{i} \leq \mathrm{pd})$. Gena lineolate and postgena imbricate. Mesoscutum tessellate-imbricate, punctation fine, dense on lateral and posterior portions $(\mathrm{i}=1-1.5 \mathrm{pd})$, sparser anteriorly and medially $(\mathrm{i}=1-3$ pd); mesoscutellum similar, evenly punctured across surface. Metanotum imbricate. Preëpisternum rugulose. Hypoepimeral area finely, densely punctate $(\mathrm{i}<0.5 \mathrm{pd})$. Mesepisternum below scrobe densely punctate $(\mathrm{i} \leq \mathrm{pd})$, interspaces imbricate. Metepisternum dorsal $1 / 3$ rugoso-carinulate, ventral portion imbricate. Metapostnotum with irregular rugae-carinulae often reaching to posterior margin, carinulae on lateral portions extending to dorsolateral slope. Propodeum posterior surfaces polished tessellate, distinct punctures on lateral surface $(\mathrm{i}=3-5 \mathrm{pd})$, irregular carinulae on posterior surface. Metasomal terga polished, finely coriarious on $\mathrm{T} 1$ anterior surface and apical impressed areas; punctation very fine ( $i=1-2 \mathrm{pd})$, apical impressed areas impunctate. Metasomal sterna coriarious and finely, sparsely punctate $(\mathrm{i}=2-4 \mathrm{pd})$.

STRUCTURE. Head slightly wider than long (length/width ratio=0.93-0.98). Eyes weakly convergent below. Clypeus $1 / 2$ below suborbital tangent. Gena narrower than eye. Hypostomal carinae subparallel. Pronotal dorsolateral angle obtuse. Pronotal ridge rounded, interrupted by sulcus. Tegula long, extended posteriorly into medially directed projection. Submarginal cells three (1rs-m present). Distal hamuli arranged 2-1-2. Inner metatibial spur pectinate, with 2-4 branches, proximal branch much longer than width of rachis. Metapostnotum narrowly rounded onto posterior propodeal surface. Propodeum with lateral carina nearly reaching dorsal margin; oblique carina fine. T2-T4 impressed areas medially about $2 / 5$ longitudinal length of basal area.

\section{Male}

MeAsuRements. Head length: $1.21-1.38 \mathrm{~mm}(\overline{\mathrm{x}}=1.28 \mathrm{~mm}, \mathrm{n}=10)$; head width: $1.23-1.36 \mathrm{~mm}$ $(\overline{\mathrm{x}}=1.28 \mathrm{~mm}, \mathrm{n}=10)$; intertegular distance: $0.79-0.94 \mathrm{~mm}(\overline{\mathrm{x}}=0.85 \mathrm{~mm}, \mathrm{n}=10)$.

Similar to female except for typical sex associated characters and as follows.

Colouration. Head and mesosoma dull metallic blue. Ventral surface of F1-F11 reddish brown. Tarsi brown to reddish brown. Metasomal terga dark brown.

PubesCENCE. Face below eye emargination with subappressed tomentum, partially obscuring surface. Metasomal terga without tomentum. Metasomal sterna with sparse, posteriorly directed setae.

Surface Sculpture. Clypeus punctures dense $(i<p d)$, evenly sized. Preëpisternum reticulate-punctate. Mesepisternum smooth, shining; punctures deep and distinct. Propodeum lateral surface densely punctate $(\mathrm{i}<\mathrm{pd})$. Metasomal terga with apical impressed areas impunctate.

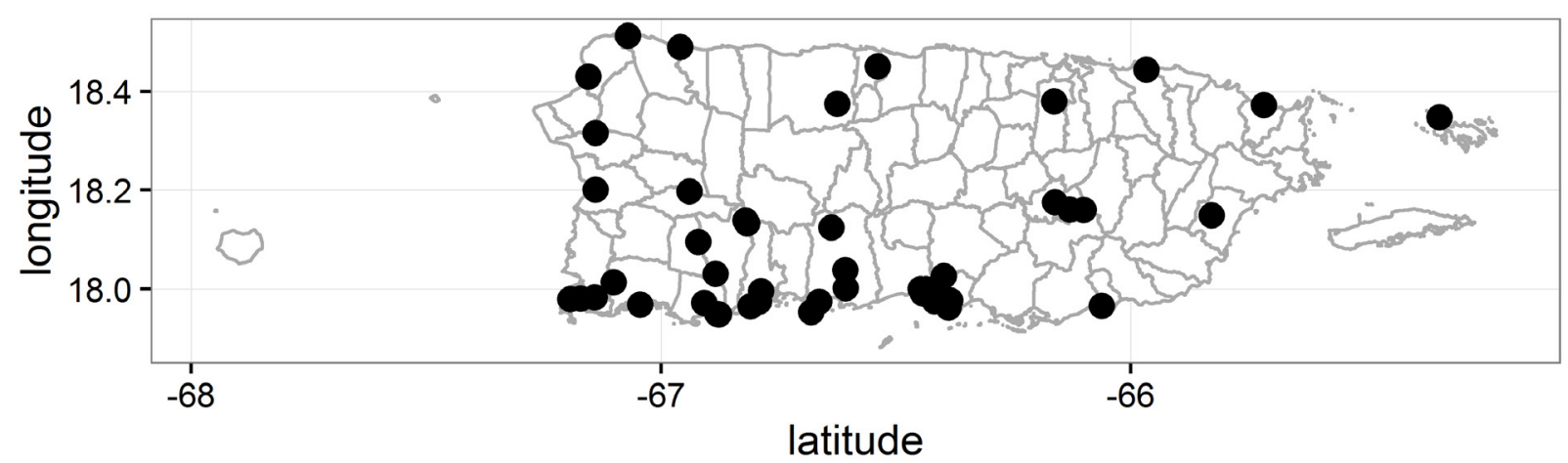

Fig. 11. Distribution map for Lasioglossum enatum sp. nov. in Puerto Rico. 
STRUCTURE. Head about as long as wide (length/width ratio=0.96-1.02). Eyes convergent below. Ratio of pedicel, F1 and F2 = 12:14:22; F2-F11 approximately $1.1-1.2 \times$ as long as wide. Metasomal terga with apical impressed areas medially less than $1 / 3$ length of basal area. T2-T3 depressed anteriorly.

Terminalia. As illustrated in Fig. 4D.

\section{Distribution}

Puerto Rico (Fig. 11), British and U.S. Virgin Islands.

\section{Biology}

Lasioglossum enatum sp. nov. has been recorded visiting the following flowers: Gundlachia corymbosa (Urb.) Britton ex Boldingh (Asteraceae), Barbieria pinnata (Pers.) Baill. (Fabaceae), Heliotropium curassavicum L. (Boraginaceae), Erythrina crista-galli L. (Fabaceae), Coriandrum sativum L. (Apiaceae), Spermacoce verticillata L. (Rubiaceae), Asparagus sp. (Liliaceae), Rosa sp. (Rosaceae), Cardiospermum microcarpum Kunth (Sapindaceae), Jacquemontia pentanthos (Convolvulaceae), Cakile lanceolata (Willd.) O.E. Schulz. (Brassicaceae), Cassine xylocarpa Vent. (Celastraceae), Ipomoea pes-caprae (L.) R. Br. ssp. brasiliensis (L.) van Ooststr. (Convolvulaceae), Coccoloba uvifera (L.) L. (Polygonaceae). Snelling (1993) also recorded this species from Schaefferia frutescens Jacq. (Celastraceae) as Lasioglossum sp. 1.

\section{DNA barcodes}

Nine individuals were successfully sequenced (BOLD Process IDs: BOWGF3186-14, BOWGF3193-14, BOWGF3192-14, BOWGF3184-14, BOWGF3183-14, BOWGF3188-14, BOWGF3185-14, NTCOL187-15 and BOWGF3187-14). DNA barcodes are distinct from L. parvum sequences from Cuba (Fig. 1), supporting the morphological characters used to recognize L. enatum sp. nov.

\section{Lasioglossum (Dialictus) monense sp. nov. urn:1sid:zoobank.org:act:7F94073B-DCB1-49A4-9956-DE505ACA07C3}

Figs $12-14$

\section{Diagnosis}

Both sexes of L. monense sp. nov. can be recognized by the following: tegula punctate and extended posteriorly to form small angle, mesepisternum punctate and metasoma reddish brown to brown. The shape of the tegula distinguishes it from all other species of L. (Dialictus) from Puerto Rico, except $L$. enatum sp nov. Other members of the L. parvum species complex, to which it belongs, share this character. Females differ from $L$. enatum sp. nov. by the more obscure supraclypeal punctures, finer mesoscutal punctation with more densely tessellate interpsaces, smooth dorsolateral slopes, and brownish red metasomal terga. The male has the mesoscutum and mesepisternum duller than in L. enatum sp. nov. due to microsculpture. In the female, the complete absence of punctures on the apical impressed margins distinguishes L. monense sp. nov. from L. parvum and L. busckiellum, which both have minute setose punctures in this area. In the male, the appressed sternal setae and dark tarsi distinguish L. monense sp. nov. from L. parvum, which has sternal setae erect and tarsi pale.

\section{Etymology}

The specific epithet monense is a Latin adjective meaning "from Mona".

\section{Material examined}

Holotype

PUERTO RICO: + , Mona Island, near Playa Pájaros, $30 \mathrm{~m}$ a.s.1., general collecting, $18^{\circ} 03^{\prime} 52^{\prime \prime} \mathrm{N}$, $67^{\circ} 52^{\prime} 06^{\prime \prime} \mathrm{W}, \mathrm{N}$. Franz leg. (NMNH). [Original label: Mona Island (PR USA), near Playa Pàjaros, 30 m, 
general collecting, $18^{\circ} 03^{\prime} 52^{\prime \prime} \mathrm{N}, 67^{\circ} 52^{\prime} 06^{\prime \prime} \mathrm{W}$, leg. N. Franz, V-18-2008 // HOLOTYPE Lasioglossum (Dialictus) monense.]

\section{Paratypes}

PUERTO RICO: Mona Island: 1 क, topotypical (JBWM); 1 †, Mona Island, 13-16 Nov. 2000, J.A. Genaro and M.A. Garcia leg. (JBWM); 1 गै, Mona Island, Punta Caigo o no Caigo, 22 Nov. 2007, J.A. Genaro leg. (JBWM); 1 §, Ulvero, road above, 11 Nov. 1992, Snelling and Torres leg. (LACM).

\section{Description}

\section{Female}

Measurements. Head length: $1.28-1.33 \mathrm{~mm}(\overline{\mathrm{x}}=1.31 \mathrm{~mm}, \mathrm{n}=3)$; head width: $1.36-1.38 \mathrm{~mm}$ $(\overline{\mathrm{x}}=1.36 \mathrm{~mm}, \mathrm{n}=3)$; intertegular distance: $0.94(\overline{\mathrm{x}}=0.94 \mathrm{~mm}, \mathrm{n}=3)$.
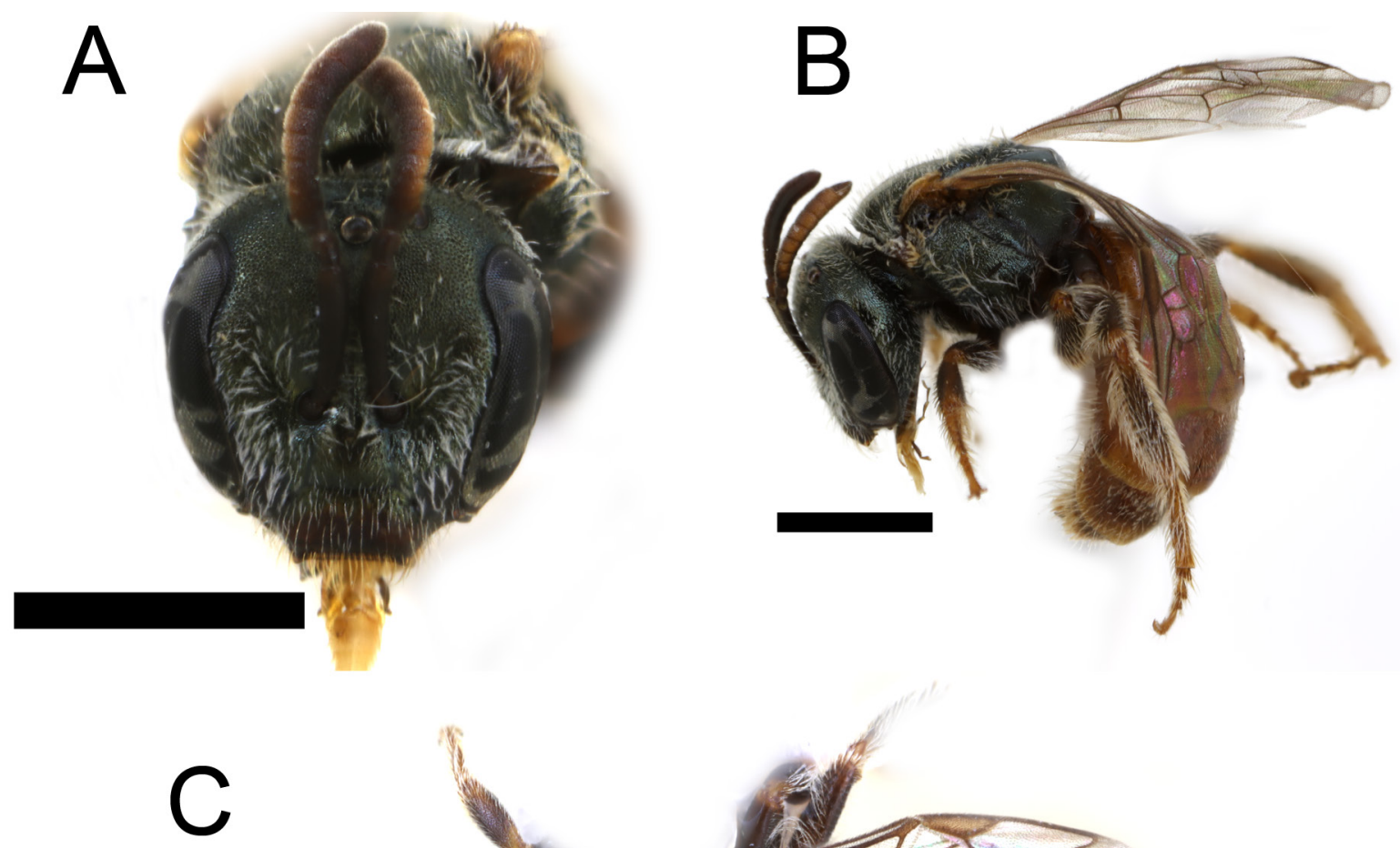

Fig. 12. Lasioglossum monense sp. nov.,, . A. Face. B. Lateral habitus. C. Dorsal habitus. Scale bars $=$ $1 \mathrm{~mm}$. 
Colouration. Head and mesosoma dull metallic golden green, except as follows. Labrum and mandible reddish brown. Clypeus distal-half dark brown. Supraclypeal area bronze. Antenna dark brown, F2-F10 with ventral surface reddish brown. Tegula dark honey-coloured. Wing membrane hyaline with dark setae, venation and pterostigma brown. Legs dark brown, tarsi reddish brown, metabasitarsus mostly brown. Metasomal terga brownish red, basal areas darker.

Pubescence. Dull white. Relatively sparse erect setae throughout, without tomentum, except sparse basolateral patches of T2-T3 and basally on T4. T1 with complete fan of appressed setae on anterior surface. T2-T4 without apical fimbriae medially, T3-T4 with sparse fine setae on apical impressed areas, sparse fimbriae laterally. Scopa well developed on hind leg and metasomal sterna.

SuRfaCe SCUlPTURE. Face imbricate, punctation fine, finer on frons, coarse on distal clypeus. Clypeus punctation sparse $(i=1-2.5 \mathrm{pd})$, finer and denser proximally, surface smooth distally $(i=2-3 \mathrm{pd})$, supraclypeal area with punctures moderately sparse $(i=1-2.5 \mathrm{pd})$, indistinct among sculptured interspaces and lower paraocular area punctation dense $(\mathrm{i} \leq \mathrm{pd})$. Upper paraocular area and frons finely reticulate-
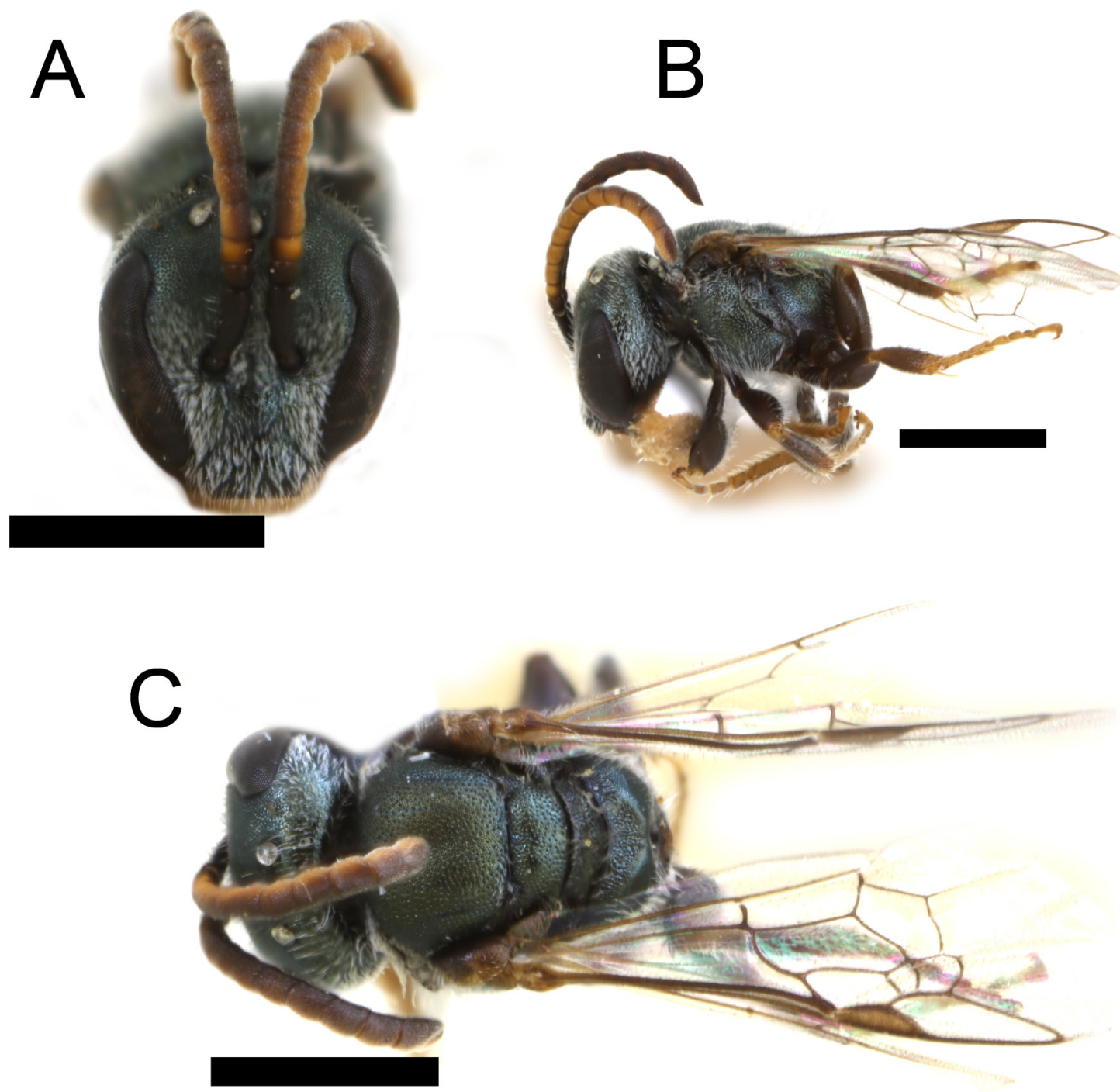

Fig. 13. Lasioglossum monense sp. nov., ठ̊. A. Face. B. Lateral habitus. C. Dorsal habitus. Scale bars = $1 \mathrm{~mm}$. 
punctate $(i<0.5 \mathrm{pd})$. Ocellocular area densely punctate $(i \leq \mathrm{pd})$. Gena lineolate and postgena imbricate. Mesoscutum tessellate, punctation fine, dense on lateral and posterior portions ( $\mathrm{i}=1-1.5 \mathrm{pd}$ ), slightly sparser anteriorly and medially; mesoscutellum similar, evenly punctured across surface. Metanotum imbricate-punctate. Preëpisternum punctate-reticulate. Hypoepimeral area finely, densely reticulatepunctate $(\mathrm{i}<0.5 \mathrm{pd})$. Mesepisternum below scrobe densely punctate $(\mathrm{i} \leq \mathrm{pd})$, interspaces imbricate, but shining below. Metepisternum dorsal $1 / 3$ rugoso-carinulate, ventral portion imbricate. Metapostnotum with irregular rugae-carinulae often reaching to posterior margin, carinulae not extending to dorsolateral slope. Propodeum posterior surfaces polished tessellate, distinct punctures on lateral surface $(i=3-5$ pd). Metasomal terga polished, finely coriarious on T1 anterior surface and apical impressed areas; punctation very fine $(i=1-2 \mathrm{pd})$, apical impressed areas impunctate. Metasomal sterna coriarious and finely, sparsely punctate $(i=2-4 \mathrm{pd})$.

STRUCTURE. Head slightly wider than long (length/width ratio=0.93-0.98). Eyes weakly convergent below. Clypeus $3 / 5$ below suborbital tangent. Gena narrower than eye. Hypostomal carinae subparallel. Pronotal dorsolateral angle obtuse. Pronotal ridge rounded, interrupted by sulcus. Tegula long, extended posteriorly into medially directed projection. Submarginal cells three (1rs-m present). Distal hamuli arranged 2-1-2. Inner metatibial spur pectinate, with 3-4 branches, proximal branch much longer than width of rachis. Metapostnotum narrowly rounded onto posterior propodeal surface. Propodeum with lateral carina nearly reaching dorsal margin; oblique carina very fine. T2-T4 impressed areas medially about $2 / 5$ longitudinal length of basal area.

\section{Male}

MeAsurements. Head length: $1.08-1.23 \mathrm{~mm}(\overline{\mathrm{x}}=1.16 \mathrm{~mm}, \mathrm{n}=2)$; head width: $1.07-1.21 \mathrm{~mm}$ $(\overline{\mathrm{x}}=1.14 \mathrm{~mm}, \mathrm{n}=2)$; intertegular distance: $0.63-0.76 \mathrm{~mm}(\overline{\mathrm{x}}=0.70 \mathrm{~mm}, \mathrm{n}=2)$.

Similar to female except for typical sex associated characters and as follows.

Colouration. Head and mesosoma dull metallic golden green. Ventral surface of F1-F11 brownish yellow. Tarsi brownish red. Metasomal terga dark brown.

PUBESCENCE. Lower paraocular area and clypeus with subappressed tomentum, partially obscuring surface. Metasomal terga without tomentum. Metasomal sterna with sparse, posteriorly directed setae.

Surface Sculpture. Clypeus punctures dense $(i<p d)$, evenly sized. Preëpisternum reticulate-punctate. Mesepisternum dull due to microsculpture; punctate. Propodeum lateral surface densely punctate $(\mathrm{i}<\mathrm{pd})$; dorsolateral slopes smooth tessellate. Metasomal terga with apical impressed areas impunctate.

Structure. Head about as long as wide (length/width ratio=1.01-1.02). Eyes convergent below. Ratio of pedicel, F1 and F2 =11:12:17; F2-F11 approximately $1.3-1.4 \times$ as long as wide. Metasomal terga with apical impressed areas medially less than $1 / 3$ length of basal area. T2-T3 depressed anteriorly.

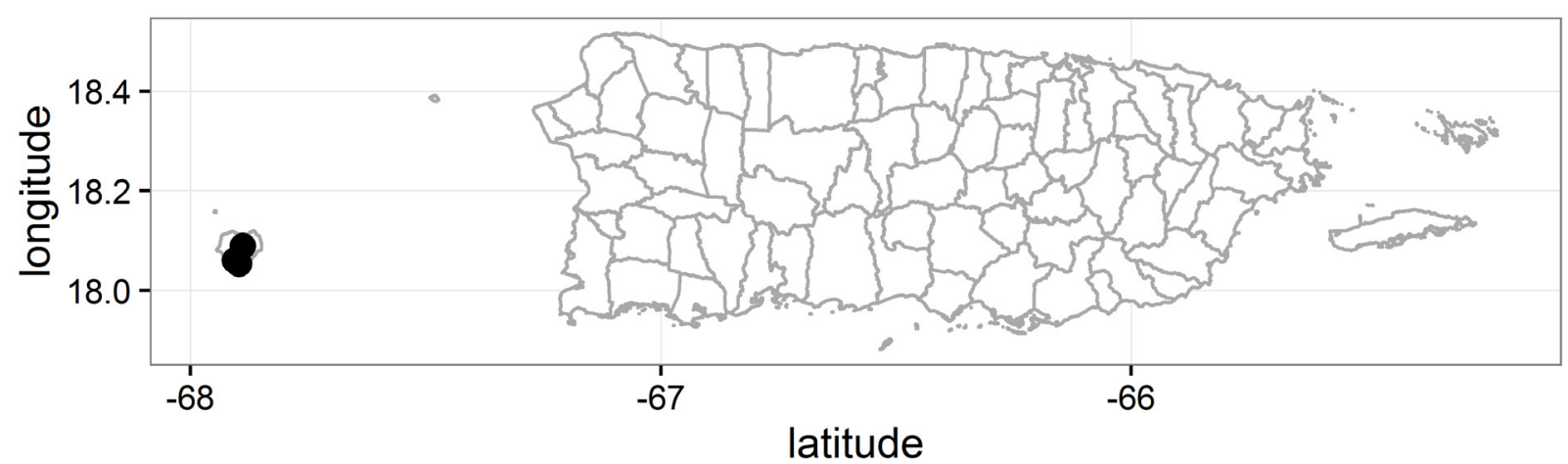

Fig. 14. Distribution map for Lasioglossum monense sp. nov. in Puerto Rico. 
Terminalia. Similar to as illustrated in Fig. 4.

\section{Distribution}

This species is apparently endemic to Mona Island (Fig. 14).

\section{Biology}

Lasioglossum monense sp. nov. visits Mammillaria nivosa Link ex Pfeiff. (Cactaceae).

\section{Remarks}

Lasioglossum monense sp. nov. could be considered a subspecies of $L$. enatum sp. nov., although use of this category can be controversial (Zink et al. 2016). The differences in microsculpture are similar to species-level differences, but it seems probable that these two species share a very recent ancestor. No specimens that could be attributable to either species have been seen from Hispaniola.

\section{Lasioglossum (Dialictus) ferrerii (Baker, 1906)}

Figs $15-17$

Chloralictus Ferrerii Baker, 1906: 263 (syntypes,, 9 , deposited at AMNH and NMNH, examined). Halictus proangularis Ellis, 1914b: 155 (holotype,, , deposited at NMNH; synonymy by George C. Eickwort (in litt., 21 Feb. 1980 to P. Alayo; see Genaro 2007; Genaro \& Franz 2008); examined images available at http://collections.nmnh.si.edu/search/ento/).

Halictus proangularis - Michener 1936: 287 (taxonomy, male description). - Wolcott 1948: 866 (checklist).

Lasioglossum ferrerii - Alayo 1973: 199; 1976: 19 (catalogue).

Dialictus ? ferrerii - Raw 1985: 2 (biology).

Dialictus ferrerii - Moure \& Hurd 1987: 100 (catalogue). — Moure 2007: 849.

Dialictus ferreri - Eickwort 1988: 236 (distribution, biology, incorrect subsequent spelling; see ICZN (1999) article 33.4).

Lasioglossum (Dialictus) ferrerii - Genaro 2004: 176 (distribution). — Genaro \& Franz 2008: 6 (distribution).

Lasioglossum (Dialictus) ferreri - Genaro 2007: 249 (distribution, incorrect subsequent spelling). Dialictus proangularis - Engel 2000: 89 (phylogeny, outgroup). — Moure 2007: 853 (catalogue).

\section{Diagnosis}

Both sexes of $L$. ferrerii are easily recognized by the combination of head relatively long (length/width ratio $=0.99-1.01$ ), mesoscutal punctures dense, except medially, mesepisternum rugose, metapostnotum short, with complete carinulae, and metasoma metallic green-blue. The large size, head shape and metallic metasoma make $L$. ferrerii very distinctive. Lasioglossum mestrei is superficially similar, but has short carinulae on the metapostnotum and lacks the rugose sculpturing on the mesepisternum.

\section{Etymology}

Baker (1906) named this species after Eduardo Ferrer.

\section{Material examined}

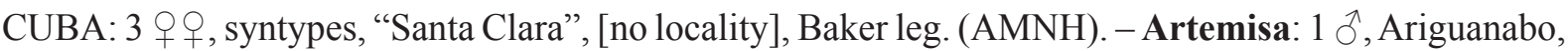
24 Nov. 1960 (SEMC). - Ciego Del Avila: 2 ô ồ, Baragua, 13 Oct. 1928, L.C. Scaramuzzo leg. (NMNH). 
- Cienfuegos: 1 $\lambda$, Cayamas, Baker leg. (AMNH). - Havana: 2 q $q$, Havana, Baker leg. (CUIC). Pinar del Rio: 1 đ, 12.5 km S of Pinar del Rio, 12-23 Sep. 1913 (AMNH).

DOMINICAN REPUBLIC: Humacao: 1 , Playa de Humacao, 11 May 1985, G.C. Eickwort leg. (CUIC). - La Altagracia: 18 우, 1 $\widehat{~}$, Nisibon, beach under coconuts, flight trap, 4-7 May 1978, G.B. Fairchild leg. (LACM). - La Vega: 1 , Jarabacoa, 17 Jul. 1986, G.C. Eickwort leg. (CUIC). - Puerto

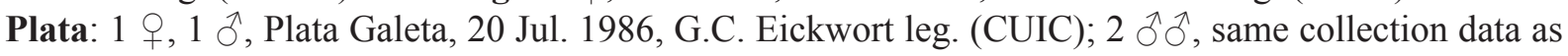
preceding, 23 Jul. 1986 (CUIC); 5 우, $4 \lesssim \curvearrowright$, same collection data as preceding, 24 Jul. 1986 (CUIC).

HAITI: Ouest: 5 ふึ̊, Etang Saumâtre, 30 Nov. 1929, J.G. Myers leg. (NMHUK).

JAMAICA: Portland: 2 q , Port Antonio, E of N shore, 1 Mar. 1907, G.B. Longstaff leg. (NHMUK). - St. Catherine: 1 , Hellshire Bay, 18 Feb. 1973, A. Raw leg. (CUIC); 1 q, Worthy Park, 2.2. mi. N

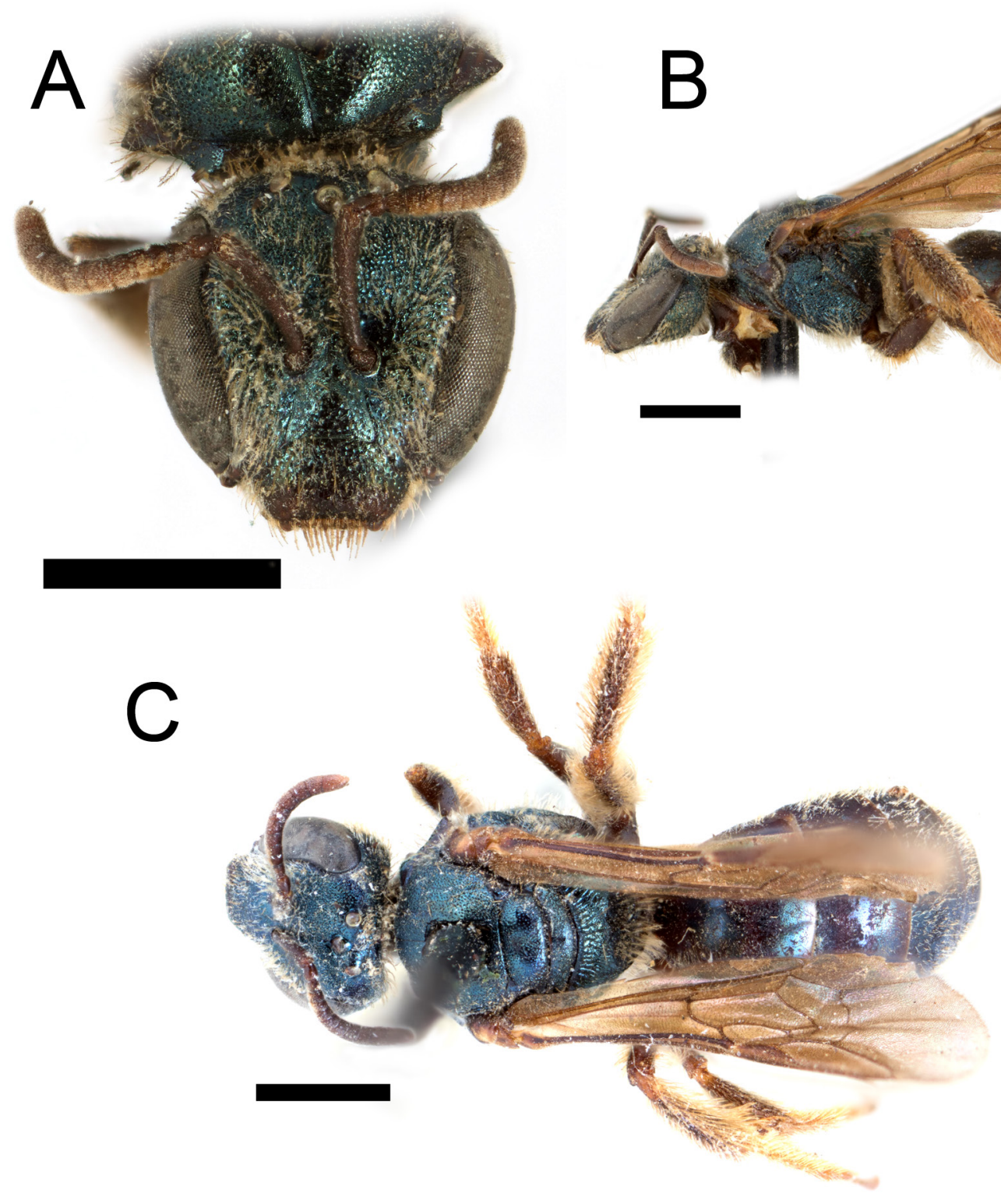

Fig. 15. Lasioglossum ferrerii (Baker, 1906), ․ . A. Face. B. Lateral habitus. C. Dorsal habitus. Scale bars $=1 \mathrm{~mm}$. 
on Camperdown Road, Malaise trap, 12 May 1969, R. E. Woodruff leg. (FSCA); 1 , same collection data as preceding, 18-25 Feb. 1970 (FSCA).

PUERTO RICO: Aguadilla: 1 q, Ramey Base, 4 Jan. 1948, P.R. Survey (NMNH); 2 q , [no locality], Jan. 1899, A. Busck leg. (NMNH). - Arecibo: 2 우, [no locality], flowers in field, 19 Dec. 1933, Anderson and Mills leg. (NMNH). - Bayamón: + , holotype of Halictus proangularis, Jan. 1899, A. Busck leg. (NMNH 23177). - Guánica: 1 సै, [no locality], 1 Dec. 1981 (FSCA). - Guayanilla: 1 , 1 ㄱ, Costa Sur, 8 Jun. 2007, J.A. Genaro leg. (JAGC). - Lajas: 2 우, Laguna Cartagena, 20 Jan. 1954, J. Maldonado Capriles leg. (JAGC); 2 우, same locality as preceding, Mar. 2008, N. Franz leg. (JAGC). - Loíza: 7 우, Islote de Juan Pérez, 28 Jun. 1986, J.A. Torres leg. (LACM). - Luquillo: 8 우, 1 đ , Balneiro de Luquillo, 9 May 1985, G.C. Eickwort leg. (CUIC); 2 우, same collection data
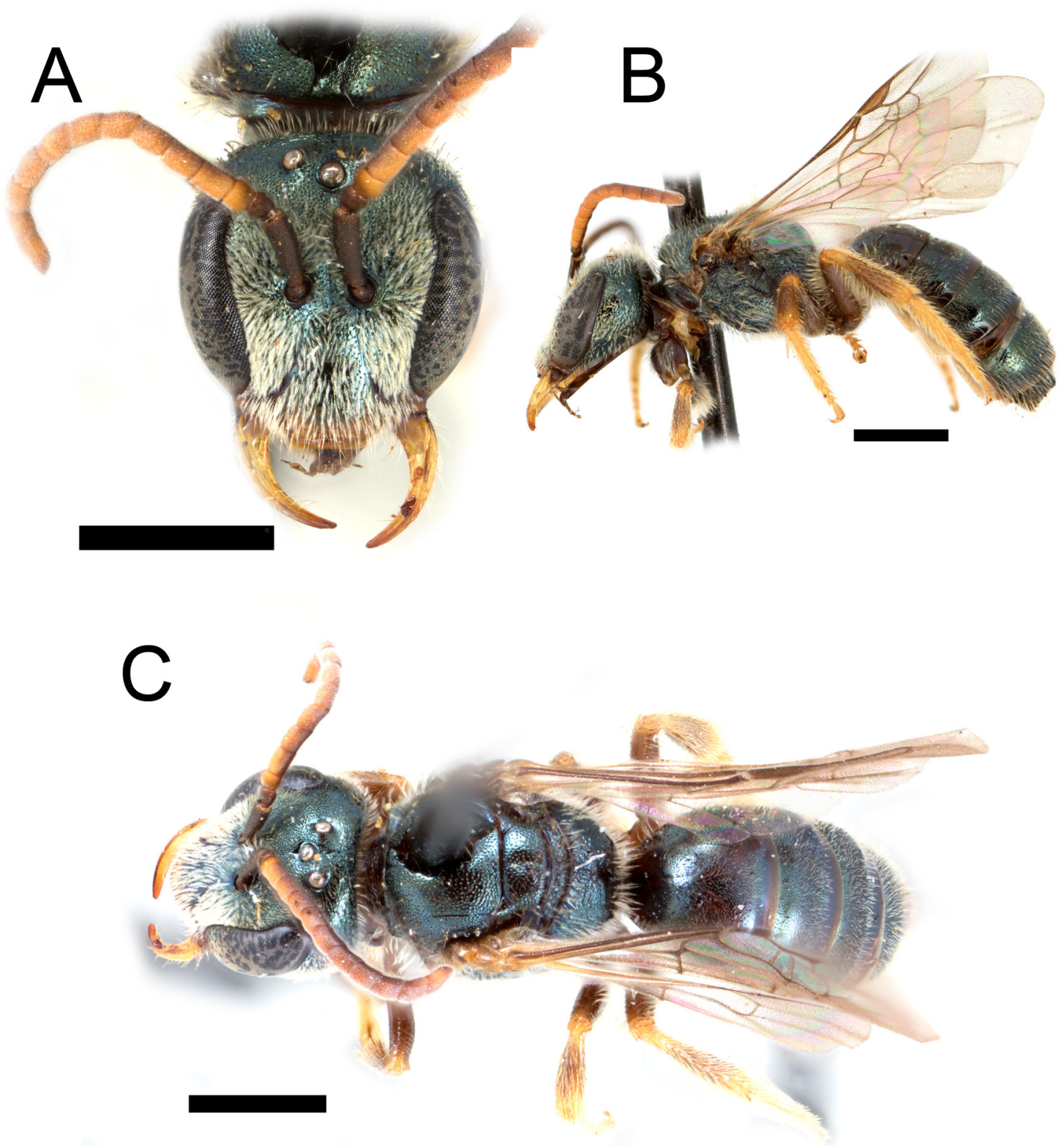

Fig. 16. Lasioglossum ferrerii (Baker, 1906), Ô. A. Face. B. Lateral habitus. C. Dorsal habitus. Scale bars $=1 \mathrm{~mm}$. 
as preceding, 10 May 1985 (CUIC); 1 \%, same collection data as preceding, 14 May 1985 (CUIC). Manatí: 2 우, [no locality], 27-29 Jun. 1915, s. coll. (AMNH). - Mayagüez: 1 +, [no locality], Jan. 1899, A. Busck leg. (NMNH); 1 + , [no locality], 15 Nov. 1981, R.A. Olivieri leg. (UPRM). - San Juan: 1 ㅇ, G.C. Eickwort homotype of H. proangularis, Rio Piedras, 24 Apr. 1926 (SEMC); 1 ○, Rio Piedras,

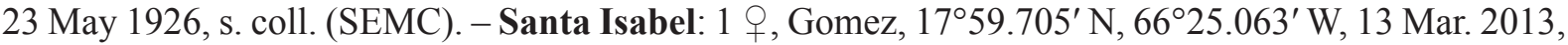
S. Prado leg. (NCSU); 2 우, Portolain, $17^{\circ} 58.632^{\prime}$ N, 66 $23.096^{\prime}$ W, 23 Apr. 2013, S. Prado leg. (NCSU: CCDB-22788 A07); 2 우우, SW River, $17^{\circ} 59.496^{\prime}$ N, 66 $26.582^{\prime}$ W, 26 Apr. 2013, S. Prado leg. (NCSU: CCDB-22788 A08, CCDB-22788 A09). - Toa Baja: 1 q, 1 Aug. 1985, J. Torres leg. (LACM); 1 ○, 2 Aug. 1985, J. Torres leg. (LACM). - Yauco: 1 ㅇ, A7 Church site, $18^{\circ} 01.830^{\prime}$ N, 66 53.042' W, elevated bee bowl, 23 May-20 Jun. 2014, S.G. Prado leg. (NCSU).

\section{Distribution}

Lasioglossum ferrerii is recorded here from Cuba, Jamaica, Hispaniola and Puerto Rico (Fig. 17). Eickwort (1988) records the "ferreri [sic] sp. group" as being present on the same four islands. Genaro (2007) and Genaro \& Franz (2008) also list the species from these same four regions. Moure (2007) did not recognize the synonymy with Halictus proangularis and only lists the country of the type locality for each name in their distributions.

\section{Biology}

Eickwort (1988) illustrated a nest of $L$. ferrerii from a "grassy upper beach area of Luquillo Beach, Puerto Rico." The nest consisted of a simple vertical burrow dividing into three main branches and reaching a maximum depth of about $24 \mathrm{~cm}$ (based on the scale provided). Six cells were illustrated, each oriented horizontally and attached to the main burrow by a short lateral tunnel or with this lateroid filled in with soil. Three multi-female nests were excavated; all occupants were inseminated, reproductive females. The number of nest-mates was few in all cases (less than 5). Eickwort (1988) concluded on this basis that $L$.ferrerii forms communal nests. The ancestral behavioural state for L. (Dialictus) is believed to be eusocial nesting (Gibbs et al. 2012b).

\section{DNA barcodes}

Three individuals were used for DNA barcoding. In each case, Wolbachia (Rickettsiales) was amplified instead of the bee. Smith et al. (2012) documented that standard barcode primers are not well suited for amplifying halictid bees.

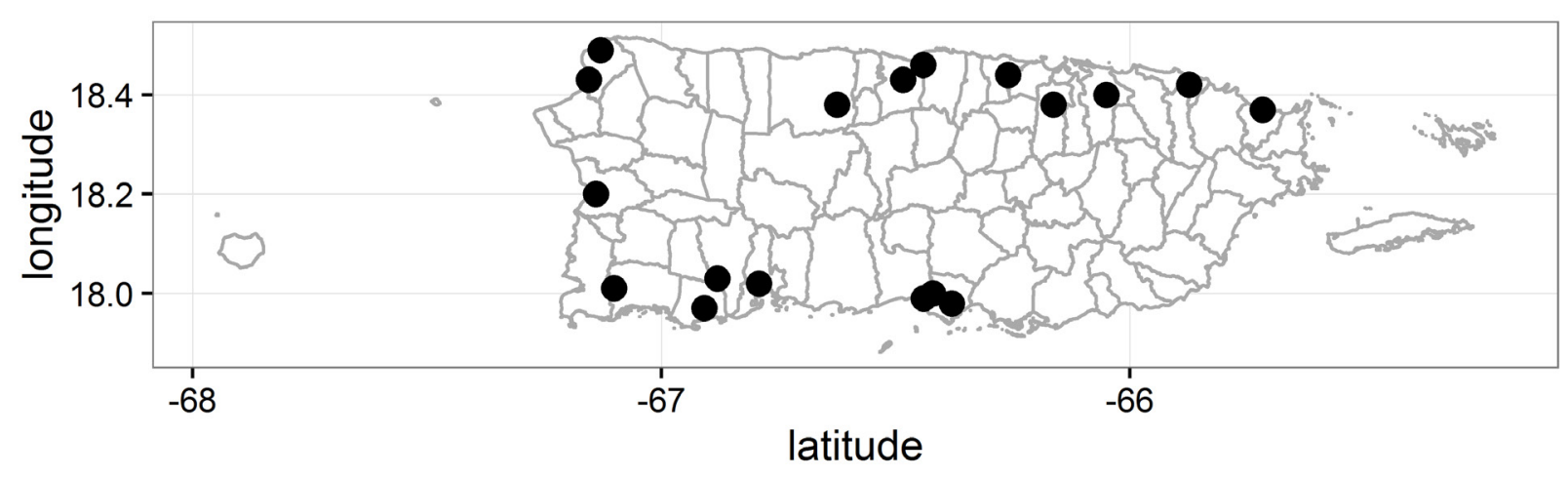

Fig. 17. Distribution map for Lasioglossum ferrerii (Baker, 1906) in Puerto Rico. 
GIBBS J., The bee genus Lasioglossum of Greater Puerto Rico

Lasioglossum (Dialictus) genaroi sp. nov. urn:lsid:zoobank.org:act:1DD459AD-2D83-424A-BDDE-68689672A028

Figs 4E, 18-20

\section{Diagnosis}

Both sexes of $L$. genaroi sp. nov. can be recognized by the combination of head wide (length/width ratio $=0.89-0.94)$, mesoscutum with coarse, relatively sparse punctation $(i=1-3 \mathrm{pd})$, tegula ovoid, mesepisternum rugose to reticulate rugose and metasoma dark brown, nearly entirely lacking in appressed tomentum. Lasioglossum genaroi sp. nov. is a quite large L. (Dialictus), being similar in size to L. ferrerii. In direct comparison, size alone is often enough to distinguish $L$. genaroi sp. nov. at a glance from L. gundlachii, L. dispersum sp. nov. and L. enatum sp. nov.

\section{Etymology}

The specific epithet is a noun in the genitive case in honour of Julio Genaro for his contributions to the study of bees and other insects in the Greater Antilles.

\section{Material examined}

\section{Holotype}

PUERTO RICO: ${ }^{+}$, Yauco, Villa Cecilia, $18^{\circ} 08.371^{\prime} \mathrm{N}, 66^{\circ} 49.230^{\prime} \mathrm{W}$, elevated bee bowl, 20 Jun.-18 Jul. 2014, S.G. Prado leg. (NCSU). [Original label: USA; PR; N18 08.371 W66 49.230 Villa Cecilia; elev. bee bowl, 20 Jun. - 18 Jul. 2014; S.G. Prado \#8 // HOLOTYPE Lasioglossum (Dialictus) genaroi Gibbs.]

\section{Paratypes}

PUERTO RICO: Ponce: 5 우, Toro Negro Forest, 2 Nov. 1992, Snelling and Torres leg. (LACM); 5 우, Vasquez, sun, $18^{\circ} 07.4616^{\prime} \mathrm{N}, 66^{\circ} 38.2638^{\prime} \mathrm{W}$, elevated bee bowl, 13 Jan.-10 Feb. 2015, S.G. Prado leg. (NCSU); 10 우, same collection data as preceding, 10 Feb.-10 Mar. 2015 (NCSU); 2 우오, same collection data as preceding, 10 Mar. -7 Apr. 2015 (NCSU); 17 우, same collection data as preceding, 17 Jun.-15 Jul. 2014 (NCSU); 2 + 9 , same collection data as preceding, 15 Jul.-12 Aug. 2014 (NCSU); 7 우오, same collection data as preceding, 9 Sep. -7 Oct. 2014 (NCSU); 4 우, same collection data as preceding, 2 Dec. 2014-13 Jan. 2015 (NCSU); 3 우, same locality as preceding, Malaise trap, 10 Feb.-10 Mar. 2015, S.G. Prado leg. (NCSU); 3 웅, 1 $\delta$, same collection data as preceding, 17 Jun.-15 Jul. 2014 (NCSU); $2 \hat{\jmath}$, same collection data as preceding, 15 Jul.-12 Aug. 2014 (NCSU); 1 q, same collection data as preceding, 12 Aug. -9 Sep. 2014 (NCSU); 1 \%, same collection data as preceding, 9 Sep. -7 Oct. 2014 (NCSU); 2 우, same collection data as preceding, 7 Oct.-4 Nov. 2014 (NCSU); 2 우, same locality as preceding, ground bee bowl, 17 Jun.-15 Jul. 2014, S.G. Prado leg. (NCSU); 1 q, same collection data as preceding, 12 Aug. -9 Sep. 2014 (NCSU); 14 우, same collection data as preceding, 7 Oct. -4 Nov. 2014 (NCSU); 2 우, Vasquez, shade, $18^{\circ} 07.4628^{\prime} \mathrm{N}, 66^{\circ} 38.1882^{\prime} \mathrm{W}$, elevated bee bowl, 10 Feb.-Mar. 10 2015, S.G. Prado leg. (NCSU); 1 ㅇ, same collection data as preceding, 7 Apr.-7 May 2014 (NCSU); 3 우, Vasquez, shade, $18^{\circ} 07.447^{\prime}$ N, 66 $38.230^{\prime}$ W, elevated bee bowl, 17 Jun. -16 Jul. 2014, S.G. Prado leg. (NCSU); 2 우, Vasquez, shade, $18^{\circ} 07.4628^{\prime} \mathrm{N}, 66^{\circ} 38.1882^{\prime} \mathrm{W}$, elevated bee bowl, 17 Jun.-16 Jul. 2014, S.G. Prado leg. (NCSU); 2 우, same collection data as preceding, 2 Dec. 2014

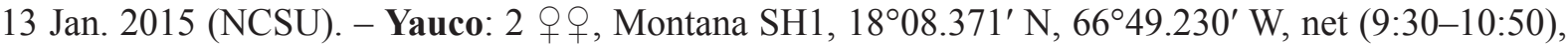
10 Jun. 2014, S.G. Prado leg. (NCSU); 1 9, Montana, sun, $18^{\circ} 07.996^{\prime} \mathrm{N}, 66^{\circ} 49.002^{\prime} \mathrm{W}$, ground bee bowl, 15 Aug.-12 Sep. 2014, S.G. Prado leg. (NCSU); 1 ㅇ, Vega, $18^{\circ} 07.724^{\prime}$ N, $66^{\circ} 53.386^{\prime}$ W, elevated bee bowl, 20 Jun. -18 Jul. 2014, S.G. Prado leg. (NCSU); 1 \&, Villa Cecilia, $18^{\circ} 08.371^{\prime} \mathrm{N}, 66^{\circ} 49.230^{\prime} \mathrm{W}$, elevated bee bowl, 15 Jan.-12 Feb. 2015, S.G. Prado leg. (NCSU); 1 \&, same collection data as preceding, 12 Feb.-12 Mar. 2015 (NCSU). 


\section{Description}

\section{Female}

Measurements. Head length: $1.39-1.58 \mathrm{~mm}(\overline{\mathrm{x}}=1.51 \mathrm{~mm}, \mathrm{n}=10)$; head width: $1.53-1.75 \mathrm{~mm}$ $(\overline{\mathrm{x}}=1.67 \mathrm{~mm}, \mathrm{n}=10)$; intertegular distance: $1.06-1.36 \mathrm{~mm}(\overline{\mathrm{x}}=1.25 \mathrm{~mm}, \mathrm{n}=10)$.

Colouration. Head and mesosoma dull metallic golden green to blue, except as follows. Labrum brown. Mandible brown with red apex. Clypeus distal half dark brown. Antenna dark brown, F9-F10 with ventral surface dark reddish brown. Tegula dark reddish brown. Wing membrane hyaline with dark setae, venation and pterostigma brown. Legs dark brown, except medio- and distitarsi reddish brown. Metasomal terga dark brown.

PubESCEnCE. Dull white. Relatively sparse erect setae throughout, without tomentum, except very narrow basolateral patches of T2-T4 immediately posterior to graduli. T1 with complete fan of appressed setae

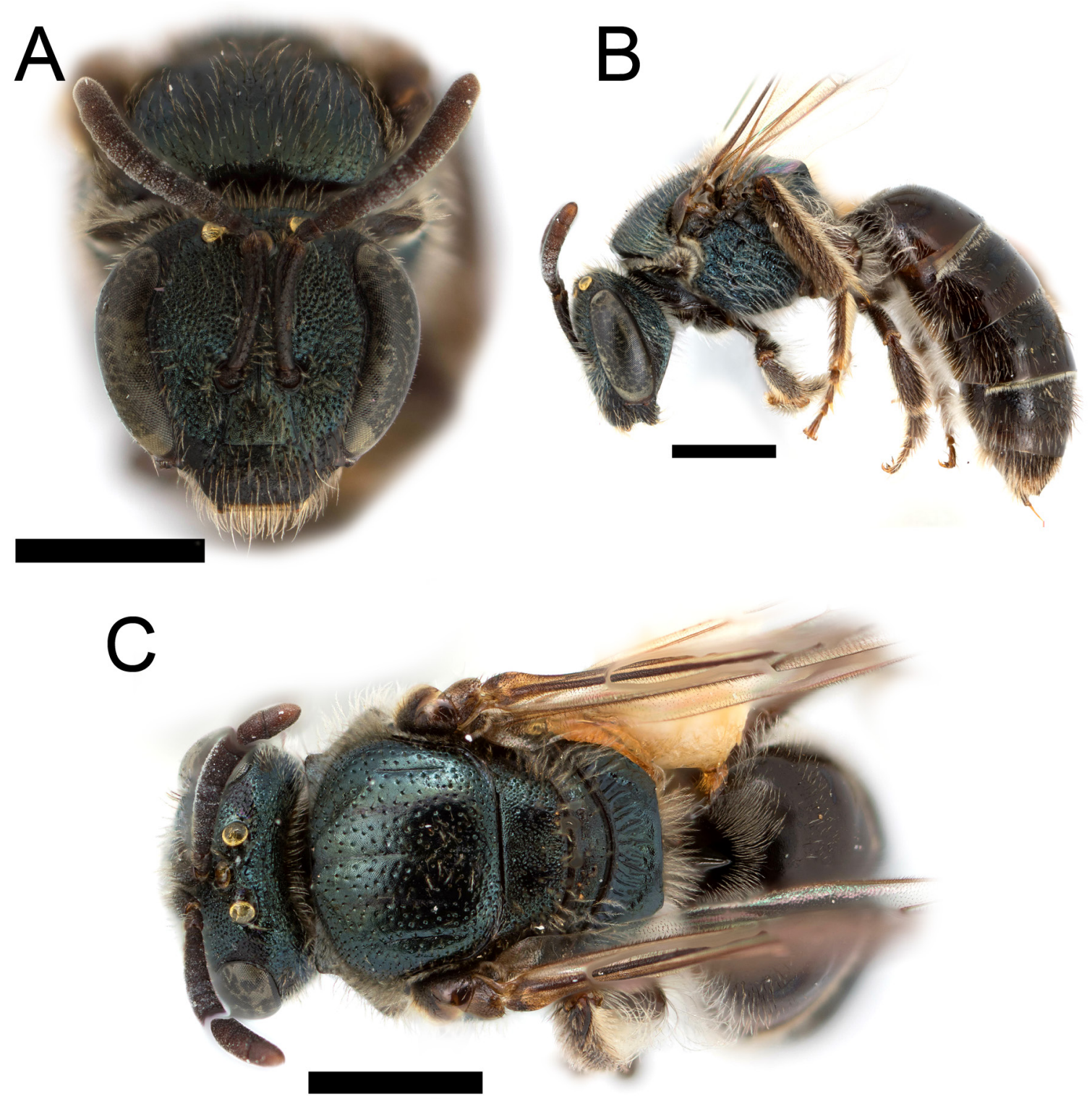

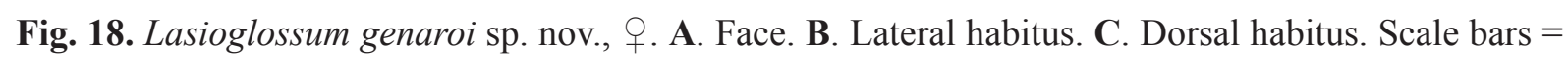
$1 \mathrm{~mm}$. 
on anterior surface. T2 without apical fimbriae, T3-T4 with only sparse fine setae on apical impressed areas. Metasomal tomentum limited to narrow band posterior to graduli, usually obscured by preceding segment. Scopa well developed on hind leg and metasomal sterna.

SuRface SCUlPtURE. Face imbricate, punctation coarse, finer on frons. Clypeus punctation sparse $(i=1-2.5 \mathrm{pd})$, surface smooth, supraclypeal area with punctures sparse $(\mathrm{i}=1-2.5 \mathrm{pd})$, and lower paraocular area punctation dense $(\mathrm{i} \leq \mathrm{pd})$. Upper paraocular area and frons reticulate-punctate $(\mathrm{i}<0.5 \mathrm{pd})$. Ocellocular area densely punctate $(\mathrm{i} \leq \mathrm{pd})$. Gena and postgena carinulate. Mesoscutum weakly imbricate, polished posteriorly, punctation moderately coarse, moderately dense on lateral and posterior portions $(\mathrm{i}=1-2 \mathrm{pd})$, sparser anteriorly and medially $(\mathrm{i}=1-3 \mathrm{pd})$; mesoscutellum with broad medial punctatereticulate region, with narrow submedial impunctate area. Metanotum imbricate. Preëpisternum rugose. Hypoepimeral area rugulose. Mesepisternum below scrobe rugose. Metepisternum dorsal $1 / 3$ rugose, ventral portion imbricate. Metapostnotum with longitudinal carinulae reaching $2 / 3-3 / 4$ distance to posterior margin, carinulae on lateral portions extending to dorsolateral slope, raised posterior margin smooth.
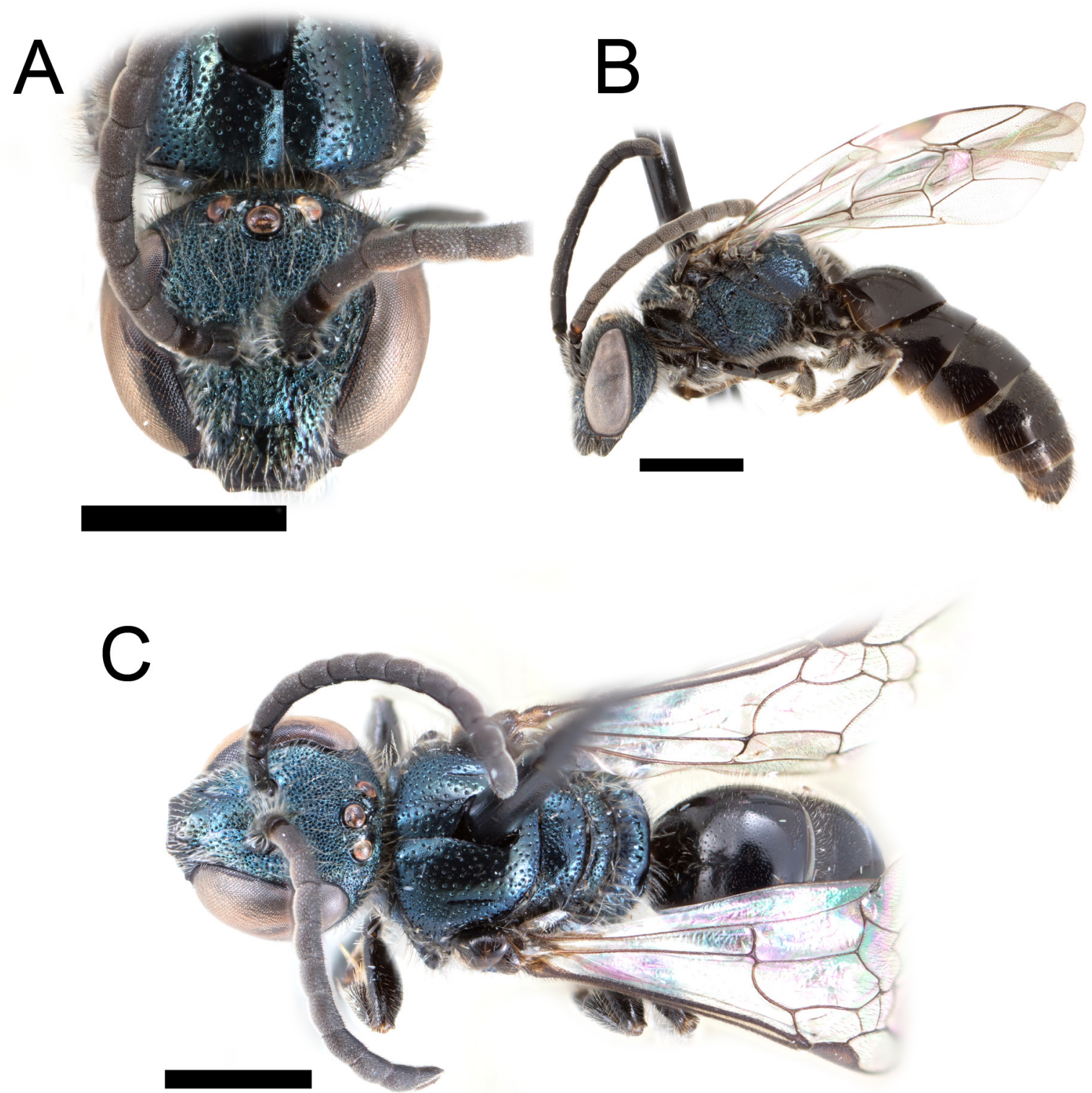

Fig. 19. Lasioglossum genaroi sp. nov., $\widehat{\partial}$. A. Face. B. Lateral habitus. C. Dorsal habitus. Scale bars = $1 \mathrm{~mm}$. 
Propodeum posterior surfaces polished tessellate, irregular sculpturing on lateral surface $(i=3-5 \mathrm{pd})$, irregular carinulae on posterior surface. Metasomal terga polished, very finely coriarious on T1 anterior surface and apical impressed areas; punctation very fine throughout, including apical impressed areas $(\mathrm{i}=1-2 \mathrm{pd})$. Metasomal sterna coriarious and finely, sparsely punctate $(\mathrm{i}=2-4 \mathrm{pd})$.

STRUCTURE. Head broader than long (length/width ratio=0.89-0.91). Eyes weakly convergent below. Clypeus $1 / 2$ below suborbital tangent. Gena narrower than eye. Hypostomal carinae subparallel. Pronotal dorsolateral angle obtuse. Pronotal ridge rounded, interrupted by sulcus. Tegula ovoid. Submarginal cells three (1rs-m present). Distal hamuli arranged 2-1-2. Inner metatibial spur pectinate, with 3-5 branches, proximal branch much longer than width of rachis. Metapostnotum slight depressed, narrowly rounded onto posterior propodeal surface. Propodeum with lateral carina reaching dorsal margin; oblique carina distinct, delimiting ventral-posterior margin on posterior and lateral margins. T2-T4 impressed areas medially nearly equal to longitudinal length of basal area.

\section{Male}

MeAsurements. Head length: $1.40-1.53 \mathrm{~mm}(\overline{\mathrm{x}}=1.47 \mathrm{~mm}, \mathrm{n}=2)$; head width: $1.49-1.66 \mathrm{~mm}$ $(\overline{\mathrm{x}}=1.58 \mathrm{~mm}, \mathrm{n}=2)$; intertegular distance: $0.99-1.23 \mathrm{~mm}(\overline{\mathrm{x}}=1.13 \mathrm{~mm}, \mathrm{n}=3)$.

Similar to female except for typical sex associated characters and as follows.

Colouration. Head and mesosoma dull metallic blue. Ventral surface of F1-F11 dark reddish brown. Tarsi brown.

PubesCEnCE. Face without tomentum. Metasomal terga without tomentum. Metasomal sterna with sparse, erect setae (1-1.5 OD).

SuRFACE SCULPTURE. Supraclypeal puncture dense $(i \leq$ pd). Mesoscutal smoother, punctation slightly sparser. Mesoscutellum sparsely coarsely punctate medially. Mesepisternum reticulate-rugose. Propodeum lateral surface punctate $(i=1-1.5 \mathrm{pd})$. Metasomal terga with apical impressed areas impunctate.

Structure. Head wider than long (length/width ratio=0.92-0.94). Eyes convergent below. Ratio of pedicel, F1 and F2=11:14:28; F2-F11 more than $1.5 \times$ as long as wide. Propodeal lateral carina not reaching dorsally to oblique carina. Metasomal terga with apical impressed areas medially about $1 / 3$ length of basal area.

Terminalia. As illustrated in Fig. 4E.

\section{Distribution}

Puerto Rico (Fig. 20). Nearly all the specimens known are from traps collected during a single study.

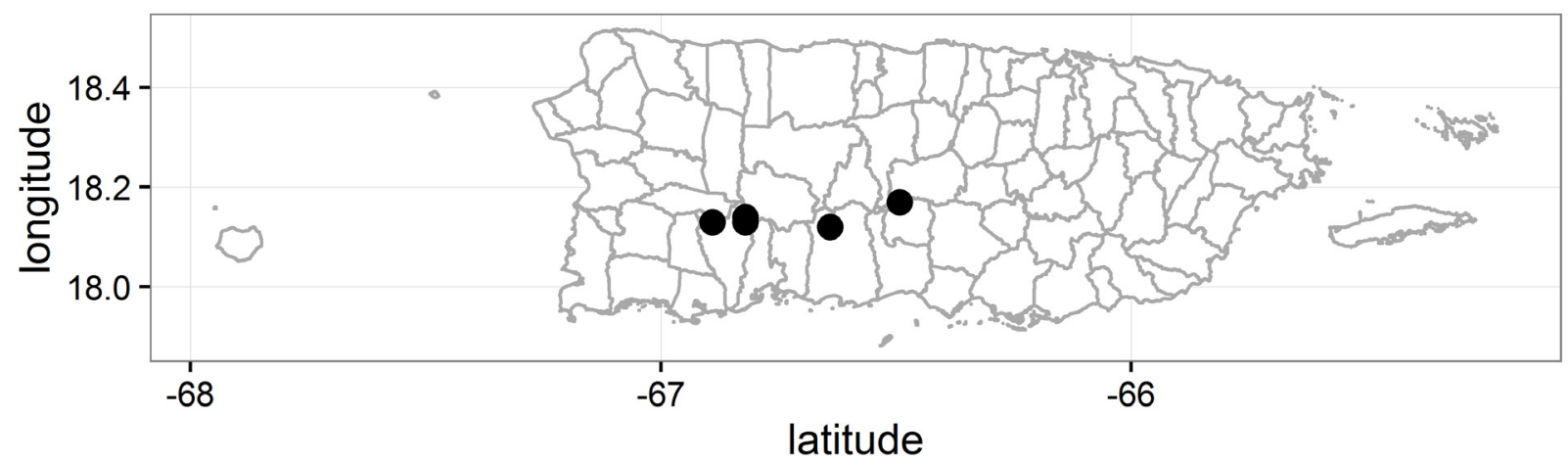

Fig. 20. Distribution map for Lasioglossum genaroi sp. nov. 


\section{DNA barcode}

One specimen was successfully amplified (BOLD Process ID: NTCOL189-15).

Lasioglossum (Dialictus) gundlachii (Baker, 1906)

Figs 4F, 21-23

Chloralictus Gundlachii Baker, 1906: 268 (syntype, ㅇ, deposited at AMNH, examined).

Lasioglossum gundlachii-Alayo 1973: 199 (catalogue); 1976: 19 (catalogue). — Rodríguez Velázquez \& Mestre Novoa 2002: 9 (distribution).

Dialictus gundlachii - Moure \& Hurd 1987: 102 (catalogue). - Moure 2007: 850 (catalogue).

Dialictus gundlachi - Eickwort 1988: 236 (distribution, biology, incorrect subsequent spelling; see ICZN (1999) article 33.4.).

Lasioglossum (Dialictus) gundlachii - Danforth 1999: 380 (phylogeny). — Danforth et al. 2003: 25 (phylogeny). - Genaro 2004: 178 (distribution); 2007: 249 (distribution). — Genaro \& Franz 2008: 6 (distribution). - Engel 2011: 66 (taxonomy). — Gibbs 2011: 90 (taxonomy). — Gibbs et al. 2012b: 930 (phylogeny).

Lasioglossum (Dialictus) mestrei (misdet.) - Genaro \& Franz 2008: 6 (distribution).

\section{Diagnosis}

Both sexes of L. gundlachii can be recognized by the metapostnotum with incomplete carinulae reaching from anterior margin to midlength, posterior half of surface smooth, mesopleuron relatively smooth, with distinct sparse punctures and mesoscutal punctures sparse between parapsidal lines. Female L. gundlachii have T1 with acarinarial fan nearly absent, only visible as small lateral patches of subappressed setae. Male L. gundlachii can be recognized by long lateral setae on posterior metasomal sterna, which protrude in horn-like tufts, distinctly visible when examining the metasoma in dorsal view. Lasioglossum mestrei also has incomplete carinulae on the metapostnotum, but it has a much larger head, denser mesoscutal punctures and metallic reflections on the metasoma.

\section{Etymology}

Baker (1906) named this species after Dr. Juan Gundlach.

\section{Material examined}

CUBA: Artemisa: 1 , Cabanas, 5-13 Sep. 1913, s. coll. (AMNH); 2 우, Laguna Ariguanabo, Jul. 1955, Zayas and Alayo leg. (LACM). - Cienfuegos: 1 ô, Ciego Montero, Palmira, Dec. 1967, P. Alayo leg. (LACM). - Guantanamo: 2 +, , El Imbano, Cuchillas de Baracoa, May 1998, R. Fernández de Arcila leg. (LACM); 4 우, Guantanamo Bay Naval Base, $19.8993^{\circ} \mathrm{N}, 75.1193^{\circ} \mathrm{W}, 5$ May 2010, S. Droege and S. Brady leg. (NMNH); 3 우, Guantanamo Bay Naval Base, $19.9166^{\circ} \mathrm{N}, 75.1408^{\circ} \mathrm{W}, 6$ May 2010, S.W.

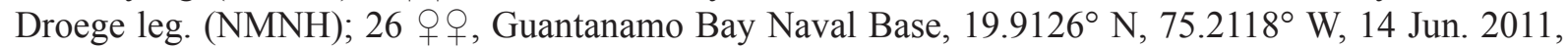
S.W. Droege leg. (NMNH). - Havana: 30 우 including syntypes, $6 \delta^{\lambda}$, s. d., Baker leg. (AMNH); 5 우 including syntypes, s. d., Baker leg. (CUIC). - Matanzas: 1 ڤึ, Sto. Tomás, P. de Zapata, s. d., J.C. Bruner and J. Acuña leg. (NMNH). - Pinar del Rio: 1 \&, Cerro Cabras, 11 Sep. 1913, s. coll. (AMNH); 2 우, Guane, 24-26 Sep. 1913 (AMNH); 2 우, Pinar del Rio, 9-24 Sep. 1913 (AMNH); 1 , San Vicente, Nov. 1955, N.L.H. Krauss leg. (SEMC); 1 + , Vinales, 7 km N, 16-22 Sep. 1913, s. coll. (AMNH); 3 우, Vinales, 24 km N, 16-22 Sep. 1913, s. coll. (AMNH). - Santiago de Cuba: 1 ○, Cristo, 3 Oct. 1915, s. coll. (AMNH). - Villa Clara: 1 P, Manicaragua, Jan. 1971, Grillo leg. (LACM).

DOMINICAN REPUBLIC: Barahona: 1 $\widehat{\jmath}$, Ciénaga, road towards Cachote, Hg and UV lights, night

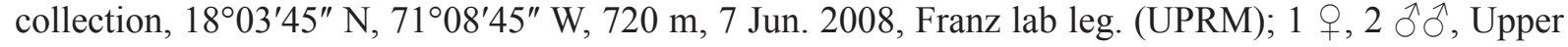


Paraiso Valley, 29 Mar. 1978, L. Masner leg. (SEMC). - Dajabon: 1 +, Villa Anacaona, 3 Jun. 1986, R. Miller and L.A. Stange leg. (FSCA). - El Seibo: 5 우, 1 ㅅ, El Seibo, 17 km N, 28 May 1985, R. Woodruff and L. Stange leg. (FSCA). - Espaillat: 1 q, Moca, Tobacco, 30 Apr. 1935, G. Russo leg. (NMNH). - Hato Mayor (?): 1 ô, San Lorenzo, 24-26 Jun. 1915, s. coll. (AMNH). - La Altagracia: 2 우, Nisibon, near cacao planting, flight trap, 5-7 May 1978, G.B. Fairchild leg. (FSCA); 1 , 1 ○, Nisibon, Malaise trap, 8-10 Jun. 1976, R.E. Woodruff and E.E. Grissell leg. (FSCA); 1 9, Rio Chacon, nr. El Naranjo, 6 Jun. 1986, R. Miller and L.A. Stange leg. (FSCA). - La Romana: 1 q, 11 Jun. 1976,

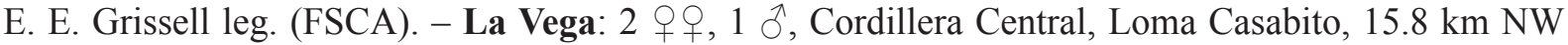

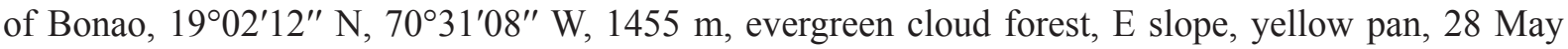
2003, J. Rawlins et al. leg. (CMNH); 13 우, Jarabacoa, 17 Jul. 1986, G.C. Eickwort leg. (CUIC); 1 q, Jarabacoa, 6 km SE, El Saito, 31 May 1986, R. Miller and L.A Stange leg. (FSCA). - Pedro Sanchez: $2 \lesssim \widehat{\jmath}$, [no locality], tobacco field, 11 Jun. 1976, E. E. Grissell leg. (FSCA). - Pedernales: 3 우, Cabo Rojo headquarters, 26 Jul. 1985, G.C. Eickwort leg. (CUIC). - Peravia: 1 , Arroyo Canas, $650 \mathrm{~m}$ a.s.l., Malaise trap, 8 Aug. 1980, A. Norrbom leg. (CMNH); 1 + , Colonia, 25 Mar. 1978, L. Masner leg.
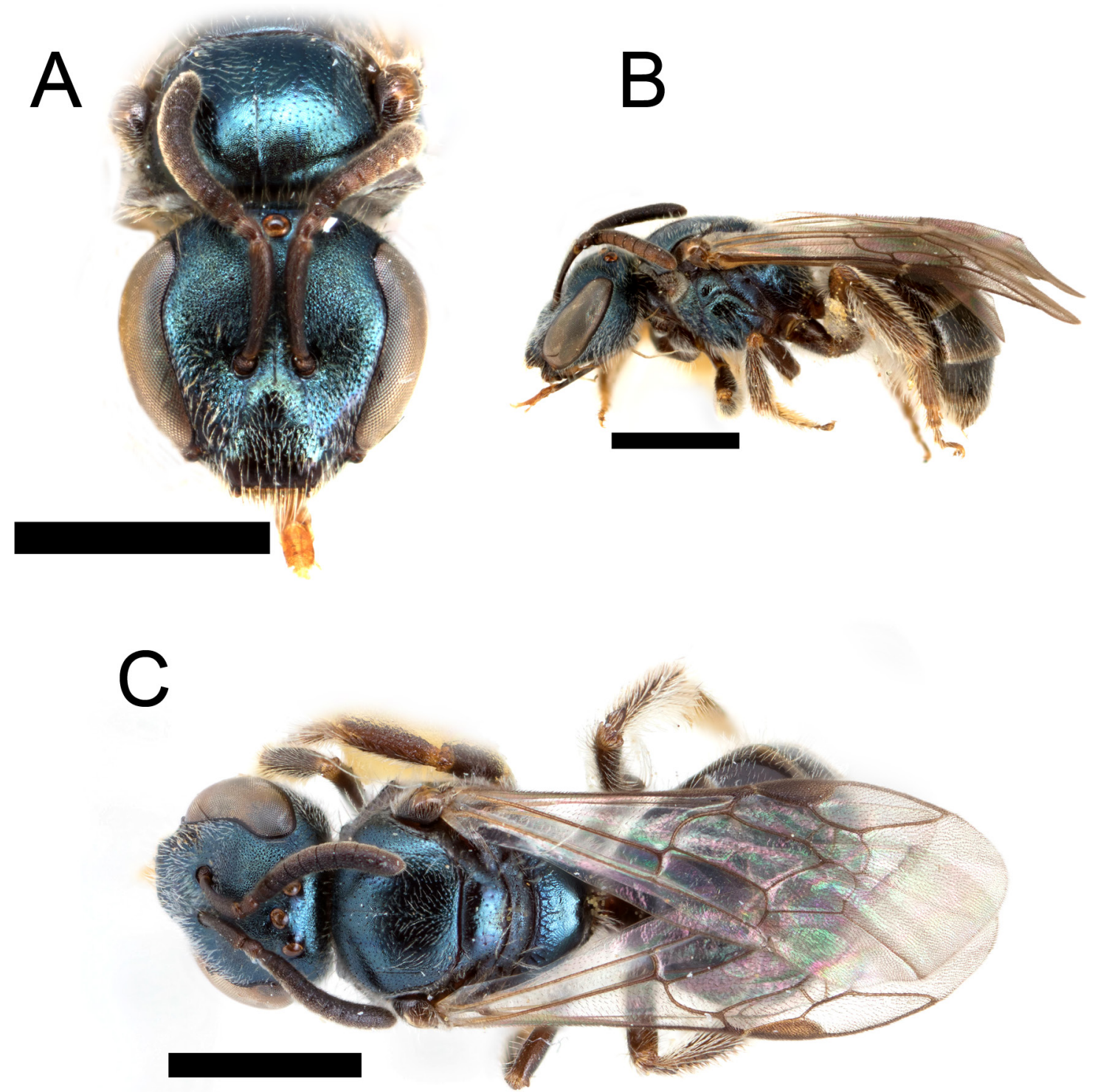

Fig. 21. Lasioglossum gundlachii (Baker, 1906), bars $=1 \mathrm{~mm}$. 
(SEMC). - Puerto Plata: 4 우, Pico Isabel de Torres, 22 Jul. 1986, G.C. Eickwort leg. (CUIC); 1 , Playa Cofresi, 30 Dec. 1984, L.A. Stange leg. (FSCA); 1 q, Playa Dorada, 28 Dec. 1984, L.A. Stange leg. (FSCA); 1 , Sosua, 25 Mar. 1985, M. Alfenito leg. (CUIC). - San Cristobal: 11 q $q, 1$ ô, Malaise trap, 16 Jun. 1986. C. Nunez leg. (FSCA); 7 우, 1 §̋, Malaise trap, 19 Jun. 1986. C. Nunez leg. (FSCA). - San Pedro de Macoris: 1 q, near San Pedor de Macoris, 16 Jul. 1917, H. Morrison leg. (NMNH).

HAITI: Ouest: 1 , Damien, 25 Nov. 1929, J.G. Myers leg. (NHMUK); 1 +, Kenscoff, 1450 m a.s.1., 14 Dec. 1929, J.G. Myers leg. (NHMUK); 1 , Leogane, 10 Aug. 1977, A. Pauly leg. (CUIC); 1 , Val. Riv. Froide, 1925, W.A. Hoffman leg. (NMNH). - Sud: 1 \&, Camp-Perrin, 1925, W.A. Hoffman leg. $(\mathrm{NMNH})$

PUERTO RICO: Adjuntas: 1 q, [no locality], 8-13 Jun. 1915, s. coll. (AMNH). - Aibonito: 1 q, [no locality], 1-3 Jun. 1915, s. coll. (AMNH). - Arecibo: 1 ô, [no locality], 1-4 Mar. 1914, s. coll. (AMNH); 1 q, 1 §, 24-26 Jun. 1915, s. coll. (AMNH). - Barceloneta: 1 , , [no locality], ex Barbieria

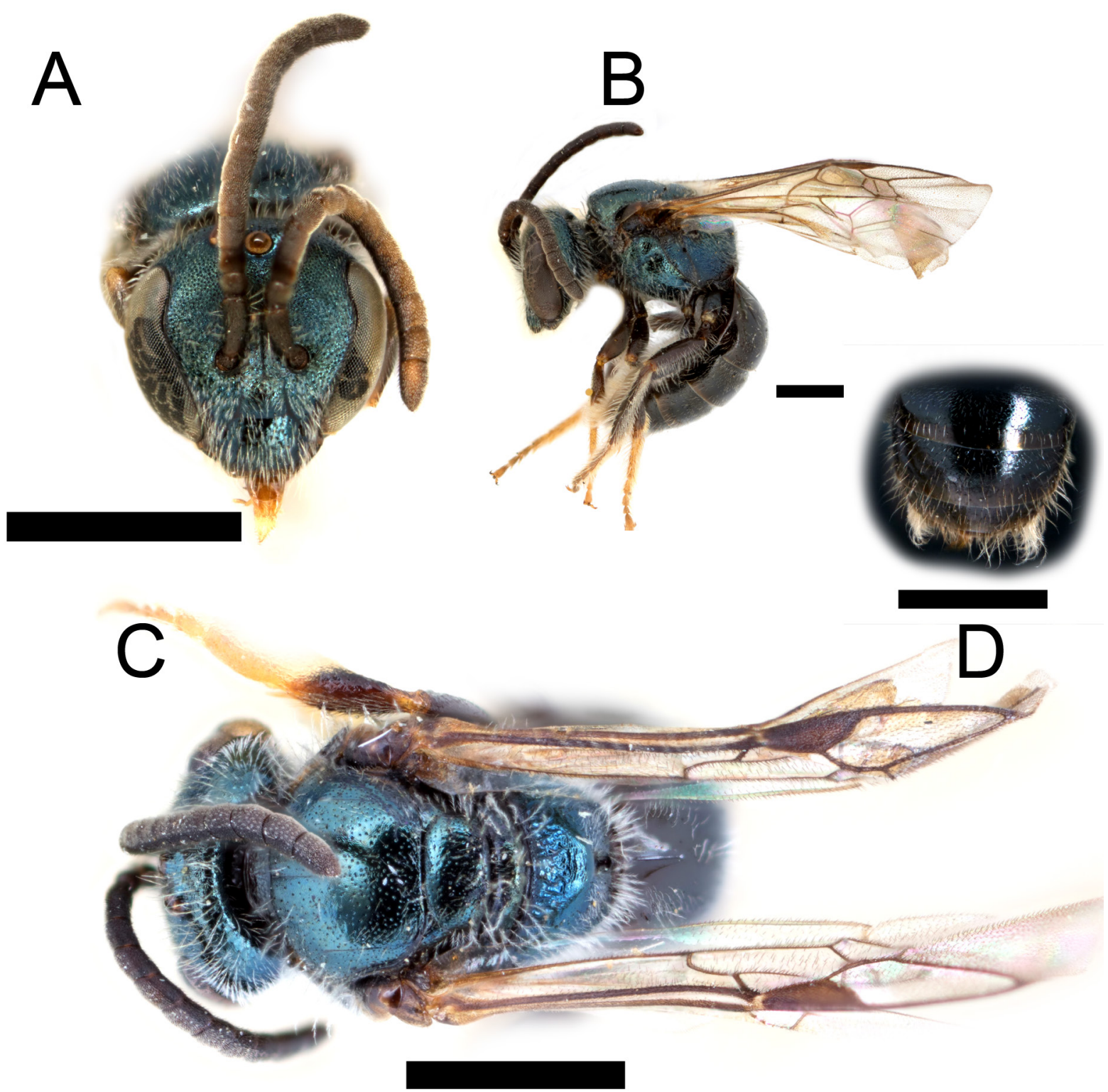

Fig. 22. Lasioglossum gundlachii (Baker, 1906), $\widehat{\partial}$. A. Face. B. Lateral habitus. C. Dorsal habitus. D. Dorsal view of metasomal apex. Scale bars $=1 \mathrm{~mm}$. 
pinnata, 21 Feb. 1933, Mills, Faxon and Anderson leg. (NMNH); 1 q, [no locality], 15 Nov. 1932, Anderson, Faxon and Mills leg. (NMNH). - Bayamón: 4 우, [no locality], ex Margarita sp., 29 Apr. 1934, Anderson and Lesesne leg. (NMNH). - Cayey: 2 우, Beatriz, 5 Nov. 1992, Snelling and Torres leg. (LACM). - Corozal: 1 + , 1 , [no locality], 1 Jan. 1920, G.N. Wolcott leg. (NMNH). - Guaynabo: 1 q, $18.36^{\circ} \mathrm{N}, 66.11^{\circ} \mathrm{W}$, Malaise trap, 20-21 May 1996, J.A. Torres leg. (LACM); 1 \%, same collection data as preceding, 29-31 May 1996 (LACM); 1 q, same collection data as preceding, 6 Jun. 1996 (LACM); 1 +, same collection data as preceding, 7-8 Jun. 1996 (LACM). - Isabela: 1 +, [no locality], ex Coriandrum sativum, 7 Apr. 1948, L.P. Martorell leg. (NMNH). - Jayuya: $2 \lesssim ð$, [no locality], 6 Jan. 1915, s. coll. (AMNH). - Luquillo: 2 đ̄ ô, Balneiro de Luquillo, 10 May 1985, G.C. Eickwort leg.

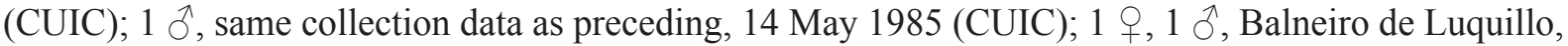
in nest \#2, 14 May 1985, G.C. Eickwort leg. (CUIC). - Manatí: 1 ㅇ, 1 Oૈ, Tortogueros Lake, 20 Nov. 1925, s. coll. (AMNH); 1 q, [no locality], 5 Mar. 1914, s. coll. (AMNH); 1 q, [no locality], 6 Apr. 1929, S.T. Danforth leg. (NMNH). - Mayagüez: 3 우, [no locality], 15-16 Feb. 1915, s. coll. (AMNH); 3 우오, [no locality], 21-23 Jun. 1915, s. coll. (AMNH); 1 đ̊, Finca Alzamora, 8 Dec. 1981, D.S. Green leg. (FSCA). - Orocovis: 1 q, [Barros, no locality], 4 Jun. 1915, s. coll. (AMNH); 1 q, Bosque Estatal Toro Negro Cordillera Central, 5 May 2008, J.A. Genaro leg. (JAGC). - Peñuelas: 19 , [no locality], Sep. 1960, M. Santiago leg. (JAGC). - Ponce: 1 ㅇ, Vasquez, sun, $18^{\circ} 07.4616^{\prime}$ N, 66 $38.2638^{\prime}$ W, elevated bee bowl, 13 Jan.-10 Feb. 2015, S.G. Prado leg. (NCSU); 2 우, same collection data as preceding, 17 Jun.-15 Jul. 2014 (NCSU); 1 q, same collection data as preceding, 9 Sep.-7 Oct. 2014 (NCSU); 6 우, same locality as preceding, Malaise trap, 17 Jun.-15 Jul. 2014, S.G. Prado leg. (NCSU); 6 우, same collection data as preceding, 15 Jul.-12 Aug. 2014 (NCSU); 2 $9+$, same collection data as preceding, 12 Aug. -9 Sep. 2014 (NCSU); 8 ㅇ, same collection data as preceding, 9 Sep. -7 Oct. 2014 (NCSU); 2 우, same collection data as preceding, 7 Oct. -4 Nov. 2014 (NCSU); 3 우, same locality as preceding, ground bee bowl, 17 Jun.-15 Jul. 2014, S.G. Prado leg. (NCSU); 1 + , Vasquez, shade, $18^{\circ} 07.447^{\prime} \mathrm{N}, 66^{\circ} 38.230^{\prime} \mathrm{W}$, ground bee bowl, 17 Jun.-15 Jul. 2014, S.G. Prado leg. (NCSU). - Rio Grande: 1 , El Verde Field Station, Road 186, REU Project, 12 Jun. 2007, Carballo-Fumero leg. (JAGC); 1 , , El Yunque, Jun. 1935, L.F. Martorell leg. (NMNH). - Guaynabo: 1 , , Sonadora, 18 Nov. 1992, J.A. Torres leg. (LACM). - San Germán: 1 + , [no locality], 23 Dec. 1962, P. and P. Spangler leg. (NMNH). - Santa Isabel: 1 q, Gomez, $17^{\circ} 59.705^{\prime} \mathrm{N}, 66^{\circ} 25.063^{\prime} \mathrm{W}, 23$ Jan. 2013, S. Prado leg. (NCSU: CCDB-22788 A12); 1 ㅇ, NW River, $18^{\circ} 0.017^{\prime} \mathrm{N}, 66^{\circ} 26.115^{\prime} \mathrm{W}, 13$ Mar. 2013, S. Prado leg. (NCSU: CCDB-22788 B01); 2 우오. SE River, $17^{\circ} 58.547^{\prime} \mathrm{N}, 66^{\circ} 25.063^{\prime} \mathrm{W}, 13$ Mar. 2013, S. Prado leg. (NCSU). - Toa Baja: 1 , Toa Baja, 2 Aug. 1985, J. Torres leg. (LACM). - Trujillo Alto: 1 , 29 Jun. 1986, J.A. Torres leg. (LACM). - Utuado: 1 9, 26 Mar. 1986, J.A. Torres leg. (LACM). - Yauco: 1 , Montana, sun, $18^{\circ} 07.996^{\prime} \mathrm{N}, 66^{\circ} 49.002^{\prime} \mathrm{W}$, ground bee bowl, 10 Oct. -5 Nov. 2014, S.G. Prado leg. (NCSU); 1 ㅇ, same collection data as preceding, 15 Jan.-12 Feb. 2015 (NCSU); 2 우, 1 ภ, Montana, same locality as preceding, net (9:30-10:50 am), 10 Jun. 2014, S.G. Prado leg. (NCSU); 1 \%, Vega,

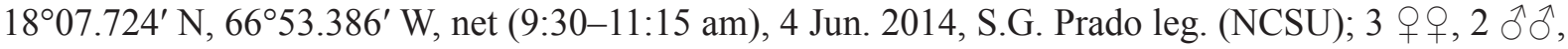
same locality as preceding, net (10:30-11:30 am) 19 Jun. 2014, S.G. Prado leg. (NCSU); 6 우, same locality as preceding, ground bee bowl, 20 Jun.-18 Jul. 2014, S.G. Prado leg. (NCSU); 2 우, same collection data as preceding, 12 Sep.-10 Oct. 2014 (NCSU); 1 क , same locality as preceding, elevated bee bowl, 20 Jun.-18 Jul. 2014, S.G. Prado leg. (NCSU); 1 q, same collection data as preceding, 15 Aug.-12 Sep. 2014 (NCSU); 1 q, same collection data as preceding, 12 Sep.-10 Oct. 2014 (NCSU); 1 q, Villa Cecilia, $18^{\circ} 08.371^{\prime} \mathrm{N}, 66^{\circ} 49.230^{\prime} \mathrm{W}$, elevated bee bowl, 15 Jan.-12 Feb. 2015, S.G. Prado leg. (NCSU); 2 우우, same collection data as preceding, 12 Feb.-Mar. 122015 (NCSU); 2 우우, same collection data as preceding, 23 May-20 Jun. 2014 (NCSU); 1 क, same collection data as preceding, 15 Aug. -12 Sep. 2014 (NCSU); 3 우, same collection data as preceding, 12 Sep.-10 Oct. 2014 (NCSU); 1 q, same locality as preceding, net (9:15-10:45 am), 4 Jun. 2014, S.G. Prado leg. (NCSU).

\section{Distribution}

Cuba, Hispaniola (Dominican Republic and Haiti), Puerto Rico (Fig. 23). 


\section{Taxonomic notes}

Two other species in the region belong to the gundlachii species group: L. liguanense (Sandhouse, 1924) from Jamaica and L. flaveriae (Mitchell, 1960) from southern Florida. The Puerto Rican material of $L$. gundlachii seems to have less dense punctation on the mesoscutum than do specimens examined from Cuba. It is possible that L. gundlachii is a senior synonym of L. flaveriae, a species known only from southern Florida and the Bahamas (Gibbs 2011). Gibbs (2011) noted differences in microsculpture between L. flaveriae and "Jamaican specimens of L. gundlachii". It is evident from examination of additional West Indian material that the Jamaican specimens examined by Gibbs (2011) were in fact L. liguanense. There are also species present in Central America (e.g., Belize) and Mexico that have close affinities to the gundlachii group (J. Gibbs, unpublished data), suggesting that this species complex may have colonized the Caribbean from this region rather than from Florida.

The description of the species L. albitarsatum (Ashmead, 1896), from Bahía Honda, Cuba, seems to match either L. gundlachii or L. mestrei. Lasioglossum albitarsatum has priority over both names. The type material of $L$. albitarsatum is evidently lost and the uncertainty in application makes it a nomen dubium.

\section{Biology}

Eickwort (1988) illustrated a nest of L. gundlachii from the same "grassy upper beach area of Luquillo Beach, Puerto Rico", where nests of $L$. ferrerii were also found. The nest was less deep than that of $L$. ferrerii, reaching a maximum depth of approximately $11 \mathrm{~cm}$. The nest was of similar construction, with eight cells attached to the primary burrows by short lateroids. Three multi-female nests were excavated. In two nests, there was a single reproductive female and a single worker. The third nest contained four inseminated females. Based on his observations, Eickwort (1988) concluded that L. gundlachii formed eusocial or semisocial nests, or occasionally communal ones.

Lasioglossum gundlachii has been recorded visiting the following flowers: Barbieria pinnata (Pers.) Baill. (Fabaceae), Coriandrum sativum L. (Apiaceae) and Margarita sp. (Euphorbiaceae).

\section{DNA barcodes}

Two specimens were sent for sequencing, but efforts on only one of these were successful (BOLD Process ID: BOWGF3194-14). The sequence is identical to a GenBank sequence (Accession: AF103965) of L. gundlachii from Puerto Rico for the $417 \mathrm{bp}$ in common (Danforth 1999). There is also a short fragment of CO1 available from L. flaveriae in Florida $(\mathrm{n}=1)$ with a maximum overlap of $531 \mathrm{bp}$ between the Florida sequence and the Puerto Rican sequences $(n=2)$. These sequences show less than $0.8 \%$ sequence divergence between the regions, which is within expected levels for a single species. Additional study

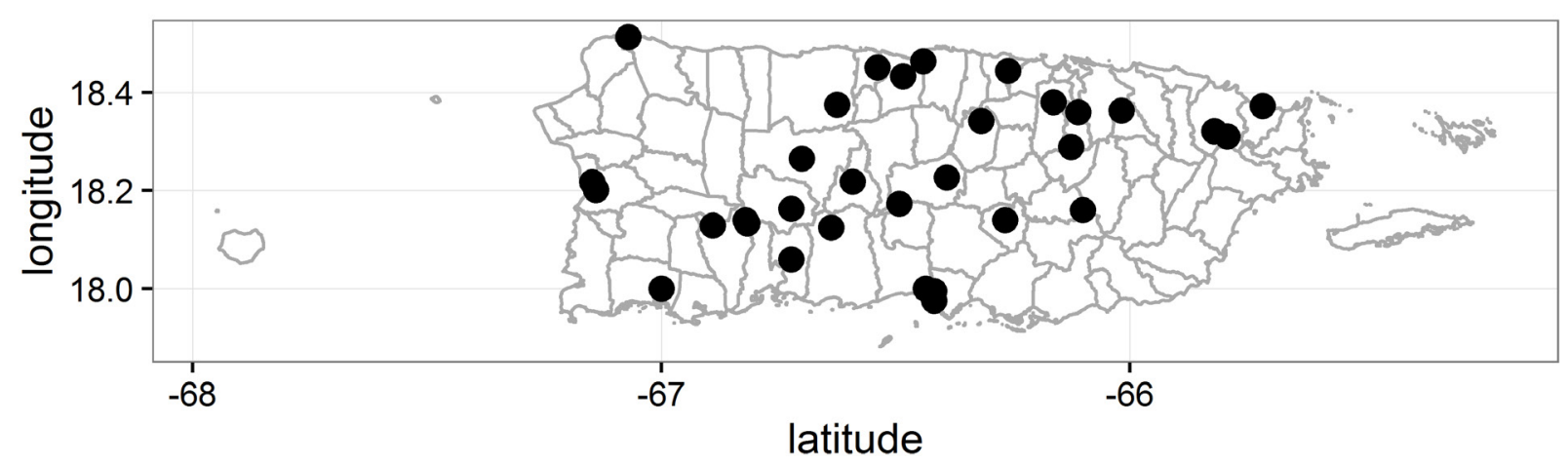

Fig. 23. Distribution map for Lasioglossum gundlachii (Baker, 1906) in Puerto Rico. 
of the gundlachii group through its range is warranted to better understand the taxonomy and historical biogeography of the group. Another species in this group, L. liguanense, is endemic to Jamaica.

\section{Subgenus Habralictellus Moure \& Hurd, 1982}

Habralictellus Moure \& Hurd, 1982: 46 (type species: Halictus auratus Ashmead, 1900, by original designation).

\section{Lasioglossum (Habralictellus) eickwortellum (Engel, 2001)}

Figs 4C, 24-26

Habralictellus eickwortellus Engel, 2001b: 35 (holotype,, , deposited at CUIC, examined).
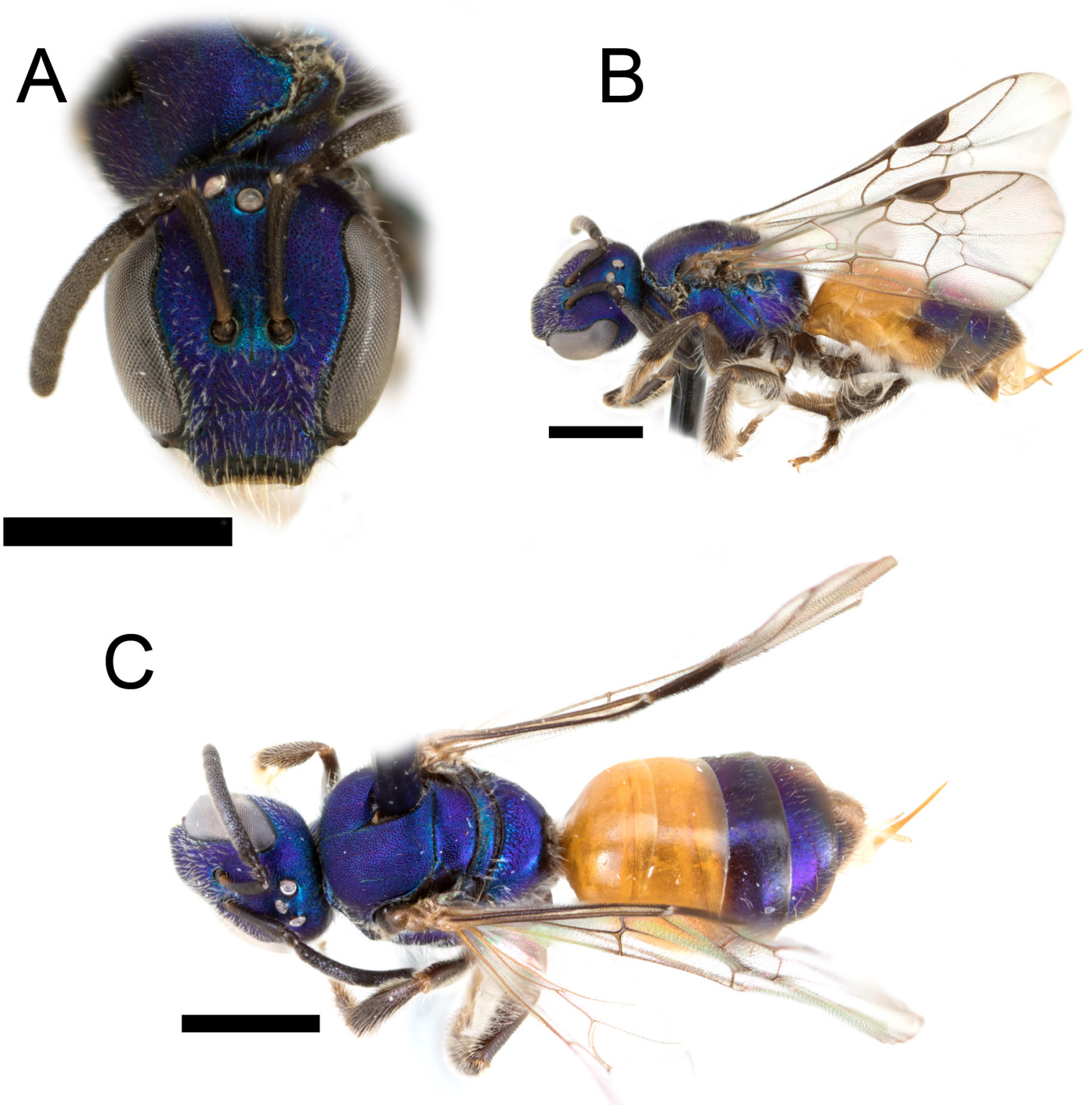

Fig. 24. Lasioglossum eickwortellum (Engel, 2001), q. A. Face. B. Lateral habitus. C. Dorsal habitus. Scale bars $=1 \mathrm{~mm}$. 
Habralictellus eickwortellus - Moure 2007: 858 (catalogue).

Lasioglossum (Dialictus) eickwortellum - Genaro \& Franz 2008: 6 (distribution); Lasioglossum (Habralictellus) eickwortellum - Gibbs 2016: 17 (taxonomy).

\section{Etymology}

Engel (2001b) named this species after Dr. George C. Eickwort.

\section{Diagnosis}

The female of L. eickwortellum is easily recognized by the bright blue-green to purple head and mesosoma, long face (length/width ratio $>1.04$ ) and metasoma with T1-T2 orange-red, T3-T6 brown with metallic reflections. The male of L. eickwortellum is similar to the female, but has more extensive
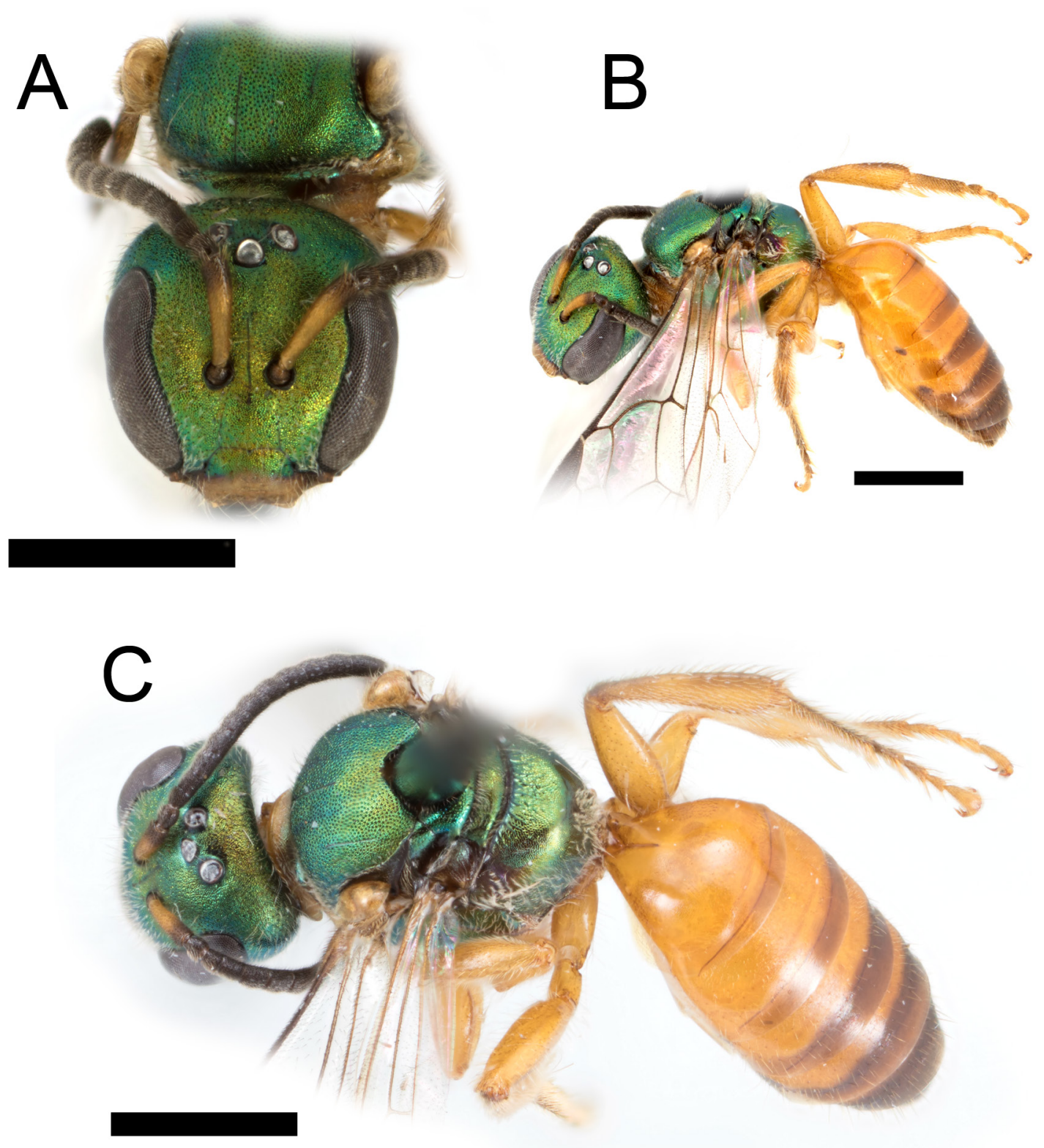

Fig. 25. Lasioglossum eickwortellum (Engel, 2001), วิ. A. Face. B. Lateral habitus. C. Dorsal habitus. Scale bars $=1 \mathrm{~mm}$. 
testaceous colouration on the metasoma, as well as yellow/testaceous colour on the clypeal distal margin; mandible and all legs are entirely yellow. Both sexes have three submarginal cells and mesoscutum with distinctive dense punctures that are closely packed over the entire surface. It is easily distinguished from L. (Habralictellus) rufopanticis, which has a wide face, sparse mesoscutal punctures, typically two submarginal cells and different patterns of metasomal colour (usually all orange-red or all brown).

\section{Material examined}

PUERTO RICO: Ponce/Jayuya: + , holotype, Cerro de Punta, Toro Negro State Forest, Cordillera Central, s. d., s. coll. (CUIC). - Ponce: 1 †, Vasquez, sun, $18^{\circ} 07.4616^{\prime} \mathrm{N}, 66^{\circ} 38.2638^{\prime} \mathrm{W}$, elevated bee bowl, 15 Jul.-12 Aug. 2014, S.G. Prado leg. (NCSU). - Rio Grande: 1 , submarginal cells 2/3, El Yunque, Rio Grande trail, 850 m a.s.1., resting on Hibiscus leaf, 2 Sep. 2007, J.E. Mercada leg. (JAGC). - Yauco:

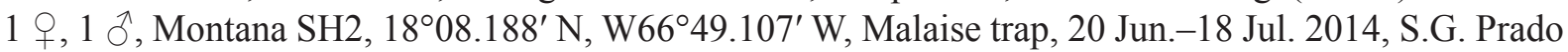
leg. (NCSU); 1 , same collection data as preceding, 12 Sep.-10 Oct. 2014 (NCSU); 1 ㅇ, Villa Cecilia, $18^{\circ} 08.371^{\prime} \mathrm{N}, 66^{\circ} 49.230^{\prime} \mathrm{W}$, net (9:15-10:45 am), 4 Jun. 2014, S.G. Prado leg. (NCSU).

\section{Description}

\section{Male}

MEASUREMENTS. Head length: $1.4 \mathrm{~mm}$; head width: $1.3 \mathrm{~mm}$; intertegular distance: $1.0 \mathrm{~mm}(\mathrm{n}=1)$. Similar to female except for typical sex associated characters.

Colouration. Head and mesosoma bright metallic green with gold reflections, except as follows. Labrum yellow. Mandible yellow with red apex, dark brown basally. Clypeus distal half yellow. Antenna dark brown, anterior face of scape yellow, F9-F10 with ventral surface dark reddish brown. Tegula honey-coloured. Wing membrane hyaline, with dark setae, venation and pterostigma dark brown. Legs entirely yellow. Metasomal terga yellow, except apical impressed areas, T6-T7 entirely and T3-T4 lateral foveae brown.

PuBESCENCE. Dull white. Relatively sparse erect setae throughout. T6-T7 and S1-S4 with relatively long setae.

SURFACE SCULPTURE. Face tessellate-granular, punctation fine and relatively sparse throughout. Clypeus punctation sparse $(i=1-2.5 \mathrm{pd})$, surface smooth distally $(i=2-3 \mathrm{pd})$, supraclypeal area with punctures moderately sparse $(i=1-2.5 \mathrm{pd})$, and lower paraocular area punctation dense $(\mathrm{i}=1-1.5 \mathrm{pd})$. Upper paraocular area and frons denser $(\mathrm{i}=1 \mathrm{pd})$. Postgena lineolate. Mesoscutum densely, consistently punctate $(\mathrm{i}=\mathrm{pd})$, sparser anteriorly and medially $(\mathrm{i}=1-3 \mathrm{pd})$; mesoscutellum similarly punctured across surface. Mesopleuron and propodeum tessellate, with only obscure, sparse punctation. Metapostnotum with short irregular rugae-carinulae reaching halfway to posterior margin. Metasomal terga polished, punctation very fine and spaser $(\mathrm{i}=1-4 \mathrm{pd})$, apical impressed areas impunctate. Metasomal sterna sparsely punctate subapically $(i=2-4 \mathrm{pd})$.

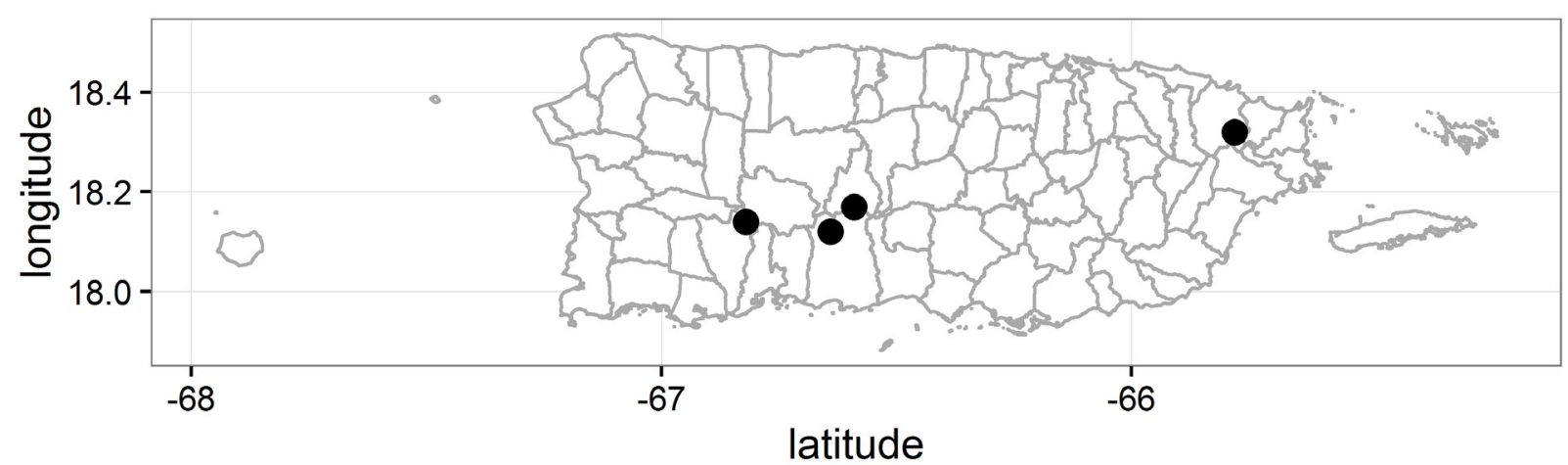

Fig. 26. Distribution map for Lasioglossum eickwortellum (Engel, 2001). 
STRUCTURE. Head slightly longer than wide (length/width ratio=1.04). Eyes weakly convergent below. Clypeus $1 / 2$ below suborbital tangent. Gena narrower than eye. Hypostomal carinae subparallel. Ratio of pedicel, F1 and F2=12:12:13; F2-F11 approximately $1.1 \times$ as long as wide. Pronotal dorsolateral angle obtuse. Pronotal ridge rounded, interrupted by sulcus. Tegula ovoid. Submarginal cells three (1rs-m present). Distal hamuli arranged 2-1-2. Inner metatibial spur pectinate, with 4-5 branches, proximal branch as wide as base of rachis. Metapostnotum narrowly rounded onto posterior propodeal surface. Propodeum with lateral carina short, reaching halfway dorsal margin; oblique carina absent. T2-T4 impressed areas medially about $2 / 5$ longitudinal length of basal area.

Terminalia. As illustrated in Fig. 4C. Gonostylus very broad, entirely covered in long setae; retrorse lobe absent.

\section{Distribution}

Puerto Rico (exclusively known from high elevation sites) (Fig. 26).

\section{Remarks}

This species was described based on a single female specimen. Three additional females and two males have been examined. The male is described above for the first time. An unusual feature of the male is the pectinate inner metatibial spur, which appears similar to that of the female. This is unusual for males of Lasioglossum, although it is present in species formerly classified as the subgenus Sudila Cameron, 1898 (Sakagami et al. 1996) (now included in Hemihalictus s. lat. (Gibbs et al. 2013)). Males of L. (Habralictellus) eleutherense (Engel, 2001) from Half Moon Cay, Bahamas (FSCA) and L. (H.) rufopanticis (Engel, 2001) from Mona Island also have pectinate inner metatibial spurs (contra Engel 2001b). This character supports uniting these species groups within L. (Habralictellus) - as does the unusual male genital capsule, which has unusually broad gonostyli and lacks a retrorse lobe (Engel 2001b: figs 1-2). Males of L. sierramaestrense (Genaro, 2001) have finely dentate inner metatibial spurs with shorter and more numerous teeth than the female spur. The holotype and paratype male of L. obamai Genaro, 2016 do not have toothed inner metatibial spurs and the genitalia look quite different from those of L. eickwortellum and L. rufopanticis, having slender elongate gonostyli and distinct retrose lobes (Genaro 2016: fig. 16). The females of all known species of L. (Habralictellus) lack the T1 appressed fan, which is present in most North American members of L. (Dialictus), but not all species (Gibbs 2009b, 2010b, 2011). The large size, bright metallic reflections, and absence of an appressed T1 fan of the adriani group species of L. (Habralictellus) is similar to the situation in some Neotropical species of Lasioglossum (J. Gibbs, unpublished data).

Two members of L. (Habralictellus) occurring in the Lesser Antilles, Lasioglossum auratum (Ashmead, 1900) and L. punctifrons (Crawford, 1914), show close affinity to L. eickwortellum, particularly in the shape of the head and the presence of three submarginal cells (Gibbs 2016). Both of these species lack the dense and distinct mesoscutal punctures and seem to have entirely dark metasoma.

Lasioglossum (Habralictellus) rufopanticis (Engel, 2001)

Figs 27-29

Habralictellus rufopanticis Engel, 2001b: 33, fig. 1 (holotype,, , deposited in LACM, examined).

Habralictellus rufopanticis - Moure 2007: 858 (catalogue).

Lasioglossum (Dialictus) rufopantex - Genaro \& Franz 2008: 6 (incorrect subsequent spelling, distribution). - Gibbs 2016: 19 (taxonomy, incorrect subsequent spelling).

Lasioglossum rufopanticis - Genaro \& Franz 2008: 3 (figure legend). 


\section{Etymology}

Engel (2001b) named this species for its reddish abdomen using the Latin words rufus and pantex. The specific epithet 'rufopanticis' is a compound noun and therefore not required to match the gender of the genus group name (see ICZN (1999) article 31.2.1).

\section{Diagnosis}

Both sexes of L. rufopanticis have the head and mesosoma iridescent green, head round, mesoscutum shiny and sparsely punctate, and submarginal cells two. They are easily distinguished from L. eickwortellum by the shape of the head (see above). They are similar to L. eleutherense, but that species has the mesosoma very dull due to distinct microsculpture and the female has distinct integumental banding on the metasoma.
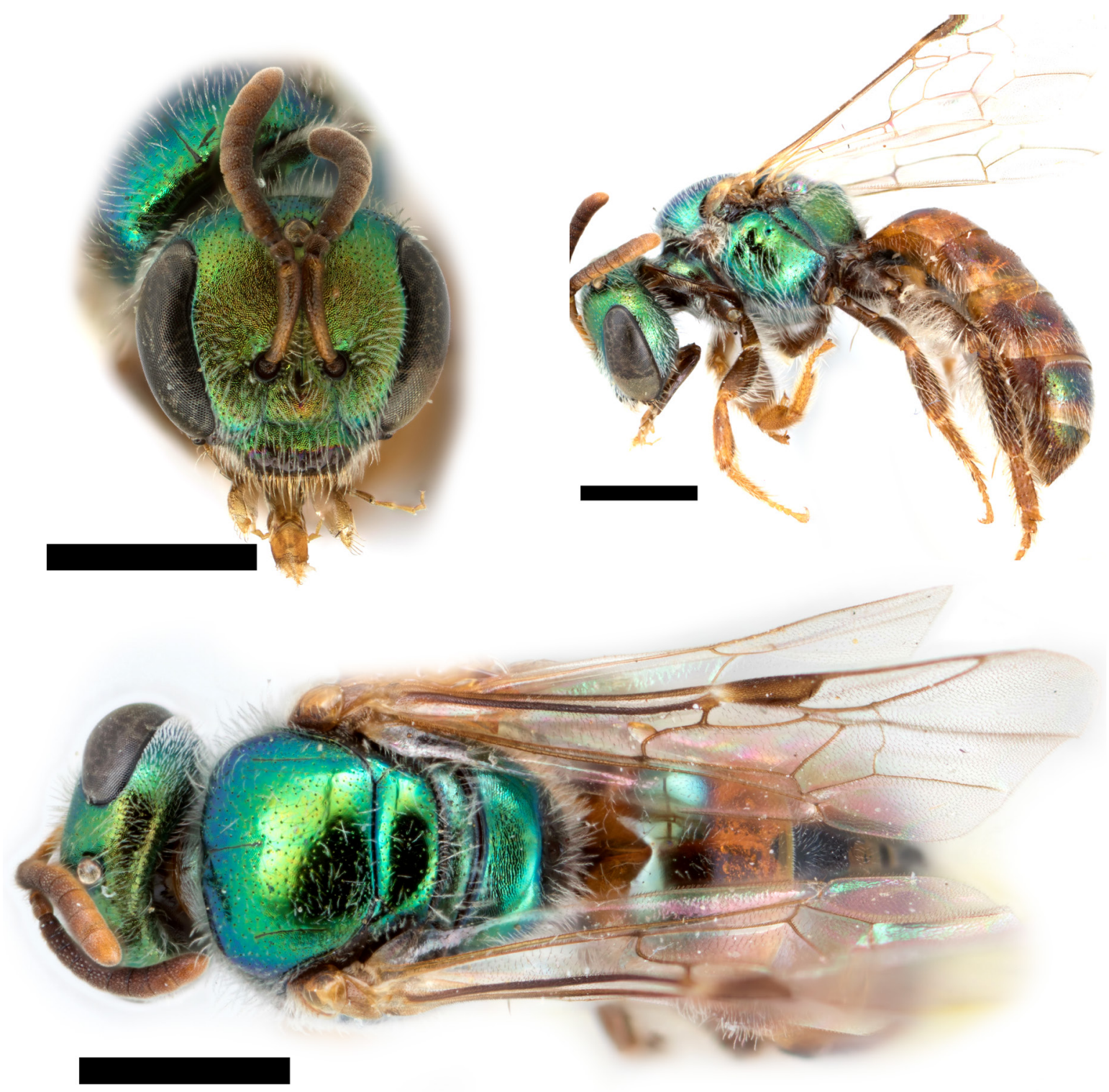

Fig. 27. Lasioglossum rufopanticis (Engel, 2001), ‥ A. Face. B. Lateral habitus. C. Dorsal habitus. Scale bars $=1 \mathrm{~mm}$. 


\section{Material examined}

PUERTO RICO: Fajardo: 2 q , Bahia Las Cabezas, 1-9 Feb. 1967, H.E. Evans leg. (NMNH). Guánica: 3 우, paratypes, 27 Apr. 1991, J.A. Torres leg. (LACM); 2 웅, Jul. 1999, J.A. Genaro leg. (JAGC); 1 q, paratype, Guánica Forest, 27 Oct. 1991, R.R. Snelling and J.A. Torres leg. (LACM). Maricao: 1 ㅇ, A77, $18^{\circ} 08.626^{\prime}$ N, 66 $68.798^{\prime}$ W, Malaise trap, 18 Jul.-15 Aug. 2014, S.G. Prado leg. (NCSU). - Mayagüez: 1 ${ }^{\lambda}$, paratype, Mona Island, road above Uvero, 11 Nov. 1992, R.R. Snelling and

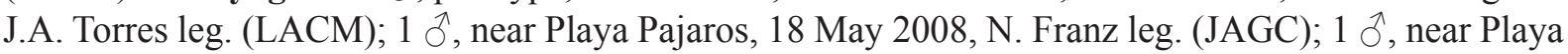

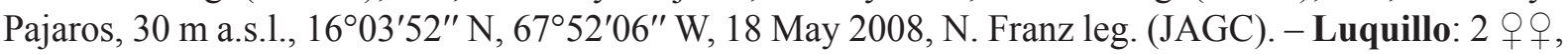
Balneiro de Luquillo, 13 May 1985, G.C. Eickwort leg. (CUIC); 1 q, same collection data as preceding, 14 May 1985 (CUIC); 1 q, [E1 Yunque] National Forest, 18 Nov. 1925, s. coll. (AMNH). - Quebradillas: 2 우, El Túnel de Guajataca, 30 May 2006, J.A. Genaro leg. (PCYU).
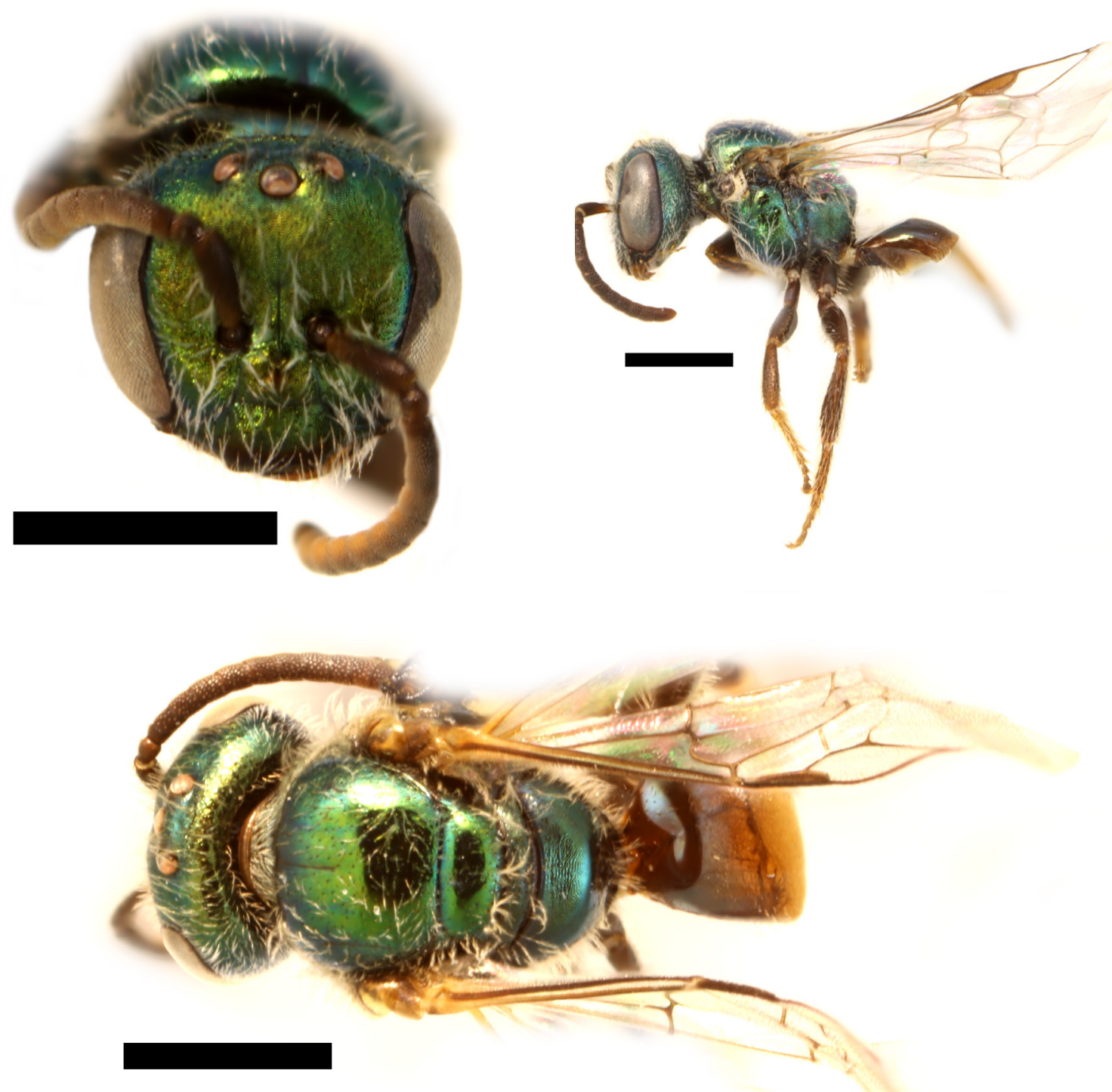

Fig. 28. Lasioglossum rufopanticis (Engel, 2001), $\widehat{o}$. A. Face. B. Lateral habitus. C. Dorsal habitus. Scale bars $=1 \mathrm{~mm}$. 
BRITISH VIRGIN ISLANDS: Guana Island: $4+q$, paratypes, hotel area, ex Capparis cynophallophora, 7 Oct. 1992, R.R. Snelling leg. (LACM); 14 우, paratypes, same collection data as preceding, 13-14 Oct. 1992 (LACM); , holotype, 14 우, paratypes, hotel area, ex Schaefferia frutescens, 26-27 Oct.

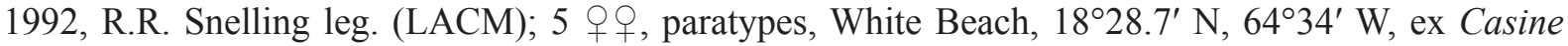
xylocarpus [sic], 2 Jul. 1993, R.R. Snelling leg. (LACM).

\section{Distribution}

British Virgin Islands (Guana Island), Puerto Rico (including Mona Island) (Fig. 29), U.S. Virgin Islands (St. John).

\section{DNA barcodes}

Two sequences are available (BOLD Process IDs: DIAL659-06 and DIAL658-06).

\section{Biology}

Observed nesting in vertical banks at Guánica and El Túnel de Guajataca, Quebradillas (Genaro \& Franz 2008). Specimens from Guana Island were collected from Capparis cynophallophora L. (Capparaceae), Schaefferia frutescens Jacq. (Celastraceae) and Cassine xylocarpa Vent. (Celastraceae) (Engel 2001b; Snelling 2005).

\section{Species removed from the checklist of Puerto Rican bees}

After examination of a large amount of material, no specimens belonging to the following three species have been seen from Greater Puerto Rico. A list of examined material for each species is provided to provide clarity on their confirmed range.

\section{Lasioglossum (Dialictus) busckiellum (Cockerel1, 1915)}

Halictus (Chloralictus) busckiellus Cockerell, 1915: 9 (holotype, ㅇ, NMNH-23176, examined).

Halictus busckiellus - Wolcott 1948: 855x (distribution, misidentification).

Dialictus busckiellus - Moure \& Hurd 1987: 93 (catalogue). — Engel 2000: 89 (phylogeny, outgroup). — Moure 2007: 847 (catalogue).

Lasioglossum (Dialictus) busckiellus - Genaro 2007: 249 (distribution, gender disagreement; see ICZN (1999) article 31.2).

Lasioglossum (Dialictus) busckiellum - Genaro \& Franz 2008: 6 (distribution, misidentification). Engel 2011: 66 (taxonomy).

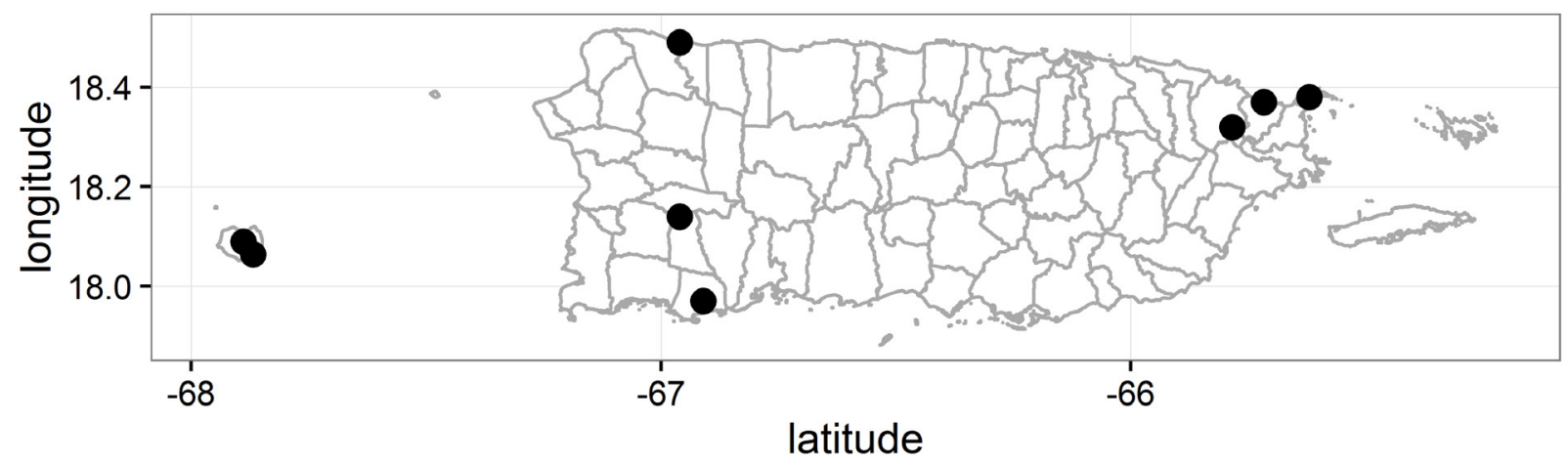

Fig. 29. Distribution map for Lasioglossum rufopanticis (Engel, 2001) in Puerto Rico. 


\section{Material examined}

DOMINICAN REPUBLIC: Independencia: 1 , 1 $\hat{\jmath}$, Los Rios, Lago Enriquillo, 25 May 1986, R.

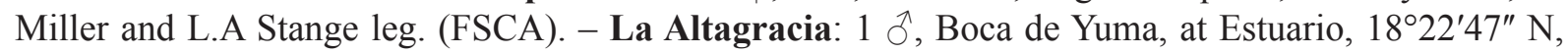

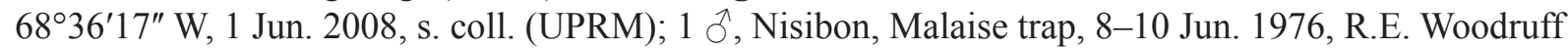
and E.E. Grissell leg. (FSCA); 2 q 9 , Parque del Este, $2.9 \mathrm{~km} \mathrm{SW}$ of Boca de Yuma, 18 21'51" N, $68^{\circ} 37^{\prime} 05^{\prime \prime} \mathrm{W}, 11 \mathrm{~m}$, semi humid dry forest, yellow pan, 28 May 2004, J. Rawlins et al. leg. (CMNH); 2 웅, Parque del Este, Caseta, Guaraguao, $4.4 \mathrm{~km}$ SE of Bayahibe, $18^{\circ} 19^{\prime} 59^{\prime \prime} \mathrm{N}, 68^{\circ} 48^{\prime} 42^{\prime \prime} \mathrm{W}, 3 \mathrm{~m}$, semi-humid forest, nr. sea, limestone, yellow pan, 26-27 May 2004, C. Young et al. leg. (CMNH). - La

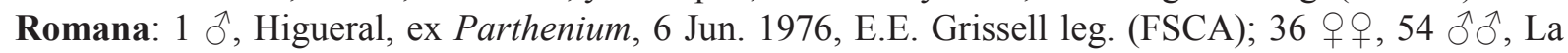

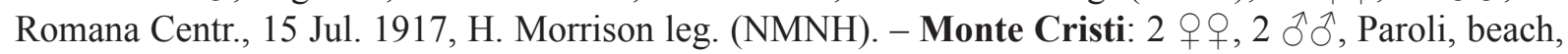
2 Jun. 1986, R. Miller and L.A. Stange leg. (FSCA). - Nacional: 3 우으, Santo Domingo (as Cuidad Trujillo), Nov. 1950, N.L.H. Krauss leg. (NMNH). - Pedernales: 1 ô, Cabo Rojo, Alcoa Rd., km 21, 24 Jul. 1985, G.C. Eickwort leg. (CUIC); 1 J , Cabo Rojo, Alcoa Rd., km 25, 26 Jul. 1985, G.C. Eickwort leg. (CUIC); 4 우, Cabo Rojo, Alcoa Rd., km 33-38, 25 Jul. 1985, G.C. Eickwort leg. (CUIC); $2 \widehat{\partial} \hat{\partial}$, Cabo Rojo headquarters, 24 Jul. 1985, G.C. Eickwort leg. (CUIC); 1 o, same collection data as preceding, 26 Jul. 1985 (CUIC); 2 우, Cabo Rojo, 21 km N, 20 Jun. 1976, E. E. Grissell and E. Marcano leg. (FSCA); 3 우, Sierra de Baoruco, Aceitillar, $25.2 \mathrm{~km}$ ENE of Pedernales, $18^{\circ} 05^{\prime} 29^{\prime \prime} \mathrm{N}, 71^{\circ} 31^{\prime} 16^{\prime \prime} \mathrm{W}$, $1272 \mathrm{~m}$ a.s.1., dense broadleaf forest, pine, yellow pan, 14 Jun. 2003, C. Young et al. leg. (CMNH). Pedro Sanchez: $2 \widehat{\partial} \hat{\partial}$, [no locality], tobacco field, 11 Jun. 1976, E.E. Grissell leg. (FSCA). - Puerto Plata: 1 + , Playa Cofresi, 30 Dec. 1984, L.A. Stange leg. (FSCA); 1 ô, Playa Sosúa, 27 Dec. 1984, L.A. Stange leg. (FSCA); 1 q, Punta Rusa, 4 Jun. 1986, R. Miller and L. Stange leg. (FSCA); 5 우,

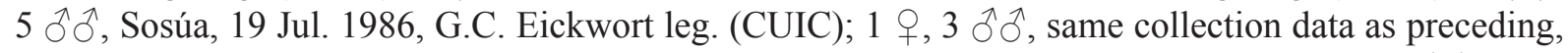

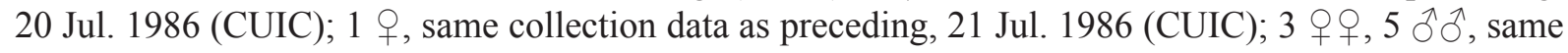
collection data as preceding, 23 Jul. 1986 (CUIC). - San Cristobal: 2 q $q$, Malaise trap, 16 Jun. 1986, C. Nunez leg. (FSCA); 4 + 9 , same collection data as preceding, 19 Jun. 1986 (FSCA).

HAITI: Artibonite: 1 , , St. Marc, 25 Oct. 1923, s. coll. (AMNH). - Ouest: 1 + , Damiens, Aug. 1977, A. Pauly leg. (CUIC); 1 क, Léogâne, 10 Aug. 1977, A. Pauly leg. (SEMC). - Sud: 4 q o, 1 Õ, Ville Formon, $31 \mathrm{~km} \mathrm{NW}$ of Les Cayes, S slope f Morne Formon, Massif de La Hotte, $18^{\circ} 20^{\prime} \mathrm{N}, 74^{\circ} 01^{\prime} \mathrm{W}$, 1405 m a.s.1., disturbed forest and fields, 7-8 Sep. 1995, Davidson et al. leg. (CMNH).

\section{Distribution}

Hispaniola (Dominican Republic and Haiti).

\section{Biology}

Lasioglossum busckiellum has been recorded from flowers of Parthenium L. (Asteraceae).

\section{Remarks}

Lasioglossum busckiellum was described from the Island of St. Domingo, now Hispaniola, probably from the Dominican Republic. This species belongs to the Lasioglossum parvum species group, which includes bees also referred to as the L. tegulare species group (Ellis 1914a; Gibbs 2009a). Wolcott (1948) recorded L. busckiellum from Puerto Rico based on specimens identified by Krombein and Sandhouse. Both L. busckiellum and L. parvum have distinctive punctation in the apical impressed areas of the metasomal T2-T3, which are absent in all L. parvum group specimens examined from Puerto Rico, including one identified as L. busckiellum by Krombein. Lasioglossum busckiellum has darker tegula than does L. parvum. Some specimens of $L$. enatum sp. nov. seem to have dark tegula suggestive of L. busckiellum. 


\section{Lasioglossum (Dialictus) mestrei (Baker, 1906)}

Augochlora parva Cresson, 1865: 171 (lectotype, $\widehat{\partial}$, deposited in ANSP; designated by Cresson (1916); lectotype not + (contra Cresson 1916); synonymy by G.C. Eickwort (in litt. in Moure \& Hurd 1987); synonymy confirmed herein; name preoccupied due to secondary homonymy with Panurgus parvus Cresson, 1865 (now also placed in Lasioglossum (Dialictus), see below)).

Chloralictus Mestrei Baker, 1906: 267 (syntypes, $q$, and ô deposited in AMNH, examined).

Augochlora parva - Dewitz 1881: 199 (distribution). — Ashmead 1900: 304 (distribution). —Friese 1902: 197 (distribution).

Chloralictus parva - Baker 1906: 259 (taxonomy, misidentification, incorrect subsequent spelling).

Halictus (Chloralictus) parva - Cockerell 1910: 489 (taxonomy).

Lasioglossum parvum - Alayo 1973: 198 (catalogue); 1976: 19 (catalogue).

Dialictus mestrei - Moure \& Hurd 1987: 112 (catalogue). — Engel 2000: 89 (phylogeny, outgroup). Moure 2007: 851 (catalogue).

\section{Material examined}

CUBA: Artemisa: 1 , Laguna de Ariguanabo, Nov. 1962, Barros-Garcia leg. (SEMC). - Cienfuegos: 1 q, Belmonte, Central Soledad, 1 Sep. 1930, R. Dow leg. (CUIC). - Havana: 3 q $ᄋ, 2$ $\widehat{\jmath}$, Calabazar,

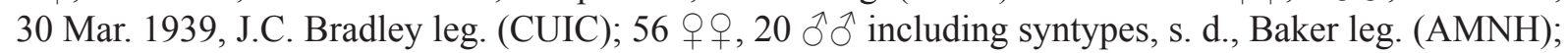
2 우, $4 \hat{\jmath} \widehat{\jmath}$, s. d., Baker leg. (CUIC); 1 , , Jan.-Feb. [unknown year], s. coll. (AMNH). - Mayabeque: 1 Ĵ, Güines, Habana, 7 Jan. 1987, J.A. Genaro leg. (LACM); 1 ㅇ, same collection data as preceding, Dec. 1987 (LACM); 4 우, same locality as preceding, Philanthus prey, 16 Dec. 1987, J.A. Genaro leg. (LACM). - Pinar del Rio: 1 , Cabanas, 5-8 Sep. 1913, s. coll. (AMNH). - Sancti Spiritu: 2 o , Zaza del Medio, 30 Sep. 1913, s. coll. (AMNH). - Santiago de Cuba: 3 우, Cristo, 3 Oct. 1915, s. coll. (AMNH); 1 §ె, botanical garden, Dec. 1994, J.A. Genaro leg. (SEMC).

DOMINICAN REPUBLIC: Dajabon: 1 , Mariano Cestero, 650 m a.s.1., Malaise trap, 13 Aug. 1980, A. Norrbom leg. (CMNH). - La Vega: 7 우, Jarabacoa, 17 Jul. 1986, G.C. Eickwort leg. (CUIC); 1 J, Jarabacoa, $600 \mathrm{~m}$ a.s.1., Malaise trap, 10 Aug. 1980, A. Norrbom leg. (CMNH). - Nacional: 2 우, Haina, Malaise trap, 20 May 1985, H.L. Dominguez leg. (FSCA); 1 ㅇ, same collection data as preceding, 30 May 1985 (FSCA); 2 우, same locality as preceding, Malaise trap, 25-30 May 1995, Woodruff and Stange leg. (LACM). - Puerto Plata: 1 , , Playa Cofresi, 30 Dec. 1084, L.A. Stange leg.

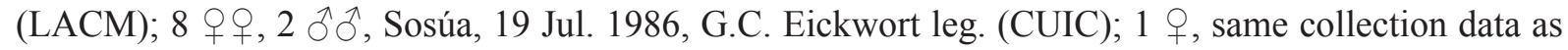
preceding, 20 Jul. 1986 (CUIC); 2 우, same collection data as preceding, 21 Jul. 1986 (CUIC); 1 , same collection data as preceding, 22 Jul. 1986 (CUIC); 4 우, $2 \hat{\mathrm{O}} \hat{\mathrm{o}}$, same collection data as preceding, 23 Jul. 1986 (CUIC); 1 q, same collection data as preceding, 24 Jul. 1986 (CUIC); 5 q, 1 กิ, Sosúa, Valley of the Caves, 20 Jul. 1986, G.C. Eickwort leg. (CUIC). - San Cristobal: 2 우, 2 o $\sigma^{\lambda}$, Malaise trap, 16 Jun. 1986, C. Nunez leg. (FSCA); 1 \&, 1 ऽે, same collection data as preceding, 19 Jun. 1986 (FSCA). - San Juan: 1 ठ, Pedro Corto, 15 km N San Juan, 21 May 1985, Woodruff and Stange leg. (LACM).

HAITI: Ouest: 1 , Bizoton, 25 Dec. 1921, s. coll. (AMNH); 2 +,+ 1 §, Carrefour, 7 Jun. 1922, s. coll. (AMNH); 1 \&, Damien, Aug. 1977, A. Pauly leg. (SEMC); 1 \%, Grand Goave, Jul. 1961, J. Maldonado C. leg. (NMNH). - Sud: 1 Ô, Aux Cayes, 15-22 Mar. 1922, s. coll. (AMNH). - Sud-Est: 1 q, Jacmel, bord de mer [seaside], 26 Aug. 1977, A. Pauly leg. (CUIC).

JAMAICA: Clarendon: 1, 3 $\widehat{\partial}$, Mason River, near Kellits, 1 Aug. 1985, G.C. Eickwort leg. (CUIC). - Portland: 1 †, $2 \widehat{\jmath}$, Somerset Falls, 31 Jul. 1988, G.C. Eickwort leg. (CUIC). - St. Catherine: 1 q, bog walk, 9 Jul. 1970, A. Raw leg. (CUIC); 1 +, 1 ô, Worthy Park, 2.2 mi N on Camperdown Road, Malaise trap, 10 May 1969, R.E. Woodruff leg. (LACM). - St. James: 1 đ, Montego Bay, 30 
Dec. 1913, s. coll. (AMNH). - Trelawney: 3 우, Clarkstown, 4.8 km E, ex Bidens pilosa, 5 Jun. 1985, C.D. Michener leg. (SEMC); 1 \%, Duanvale, 10 Aug. 1985, G.C. Eickwort leg. (CUIC); 1 + , 1 §, Good Hope, 9 Aug. 1985, G.C. Eickwort leg. (CUIC); 1 ổ, Rio Bueno, 4.8 km S, 7 Jun. 1985, C. D. Michener

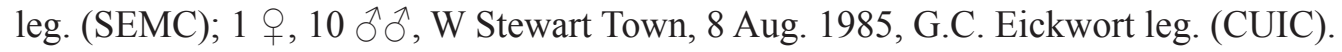

\section{Distribution}

Cuba, Hispaniola (Haiti and the Dominican Republic), and Jamaica.

\section{Biology}

Lasioglossum mestrei has been recorded from flowers of Bidens pilosa L. (Asteraceae).

\section{Remarks}

There has been some recent and past confusion regarding this species, such that some individuals recently reported to be $L$. mestrei are misidentified specimens of $L$. gundlachii. Historical records may be due to confusion over the secondary homonymy of the two Cresson (1865) names Augochlora parva and Panurgus parvus. It was first recorded from Puerto Rico as Augochlora parva by Dewitz (1881). Baker (1906) treated A. parva and P. parvus as if they belonged to the same species, which was accepted by later authors (Cockerell 1910; Alayo 1973, 1976). The lectotype designations for these two names by Cresson (1916) invalidates this interpretation. Some records of $L$. mestrei from Puerto Rico may be derived from specimens of L. enatum sp. nov. due to the earlier use of the Cresson names.

\section{Lasioglossum (Dialictus) parvum (Cresson, 1865)}

Panurgus? parvus Cresson, 1865: 175. (lectotype, ${ }^{\lambda}$, deposited in ANSP, designated by Cresson (1916), examined). [Label data: Cuba. // 95 // §ै// LectoTYPE 2182.]

Panurgus parvus - Dewitz 1881: 197 (catalogue). — Gundlach 1887: 152 (distribution). — Dalla Torre 1896: 182 (catalogue). — Ashmead 1900: 303 (catalogue). — Gowdey 1926: 98 (checklist).

Halictus parvus - Cockerell 1898: 185 (taxonomy, characters); 1915: 9 (comparative taxonomy).

Panurgus? parvus - Friese 1902: 197 (catalogue).

Dialictus parvus - Cockerell 1904: 235 (taxonomic position). - Sandhouse 1923: 193 (catalogue). — Moure \& Hurd 1987: 120. — Eickwort 1988: 236 (biology, distribution). — Engel 2000: 89 (phylogeny, outgroup). - Moure 2007: 853.

Chloralictus parvus - Baker 1906: 259 (taxonomic revision, misidentification in part).

Halictus (Chloralictus) parvus - Krombein 1953: 20 (distribution).

Lasioglossum parvum - Alayo 1973: 198; 1976: 18 (catalogue).

Lasioglossum parvus - Rodríguez Velázquez \& Mestre Novoa 2002: 9 (checklist, incorrect spelling: gender disagreement).

Lasioglossum (Dialictus) parvus - Genaro 2004: 178 (distribution, incorrect spelling: gender disagreement).

Lasioglossum (Dialictus) parvum - Genaro 2007: 249 (distribution). — Gibbs 2010a: 2 (taxonomy). — Engel 2011: 66 (taxonomy).

\section{Material examined}

CUBA: "Santa Clara": 1 , [no locality], s. d., Baker leg. (AMNH). - Camaguey: 4 $q$, [no locality],

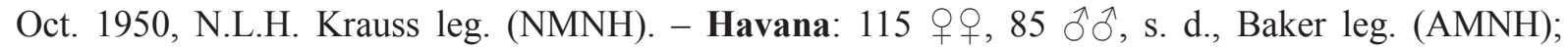

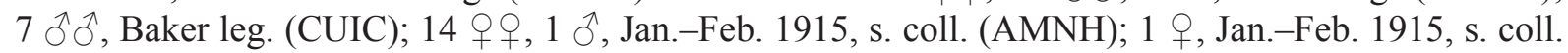
$(\mathrm{NMNH}) ; 1$, Dec. 1955, N.L.H. Krauss leg. (SEMC). - Matanzas: 1 , , Abra Figueroa Yumuri Mts, Jun. 1970, s. coll. (SEMC); 1 + , Playa Larga, Cienaga de Zapata, Jun. 1965, P. Alayo leg. (SEMC). - 
Pinar del Rio: 11 q $q$, Guane, 1918, W.M. Mann leg. (NMNH); 2 q , Guane, 24-26 Sep. 1913, s. coll. (AMNH); 1 , , La Palma, Nov. 1955, N.L.H. Krauss leg. (SEMC); 1 , , Mariel, Jun. 1968, P. Alayo leg. (SEMC); 1 q, Vinales, 7 km N, 16-22 Sep. 1913, s. coll. (AMNH); 3 우, Vinales, 7 km N, 20 Nov.

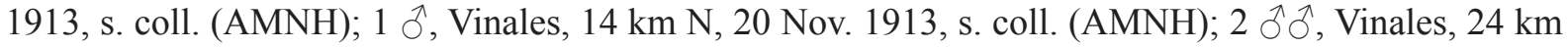
N, 16-22 Sep. 1913, s. coll. (AMNH). - Sancti Spiritu: 1 , Zaza del Medio, 30 Sep. 1913, s. coll. (AMNH). - Santiago De Cuba: 1 +, Cristo, 3 Oct. 1915, s. coll. (AMNH); 1 , , Siboney, sea level, 11 Dec. 1995, L. Masner leg. (PCYU).

\section{Distribution}

Bahamas (North and South Bimini, San Salvador), Cayman Islands, Cuba (Moure \& Hurd 1987; Genaro 2007).

\section{Remarks}

The Lasioglossum parvum species group is widespread in the New World, and includes members of the L. tegulare species group (Danforth 1999; Danforth et al. 2003; Gibbs 2009a; Gibbs et al. 2012b). The parvum group is common in the Greater Antilles. Lasioglossum busckiellum from Hispaniola (see above) and L. gemmatum (Smith, 1853) from Jamaica (= L. bruesi (Cockerell, 1912) syn. nov., $=$ L. jamaicae (Ellis, 1914) syn. nov.), L. monense sp. nov. and L. enatum sp. nov. all belong to this group. The name $L$. parvum has undoubtedly been applied too broadly in the past to include multiple species, probably including most of the above, as well as possibly undescribed species in this complex from Cuba, Jamaica and the Bahamas (Gibbs, unpublished data). Dewitz (1881) and Gundlach (1887) recorded it from Puerto Rico as Panurgus parvus, but the bee diversity of the region was not well known at that time. All specimens belonging to the parvum group examined from Puerto Rico were L. enatum sp. nov. or L. monense sp. nov. from Mona Island. Several specimens of the parvum group with two submarginal cells in at least one wing were examined from the Bahamas and Cayman Islands. Record details for these specimens are as follows:

BAHAMAS: Long Island: 1 + , Clarence Town, 13 Mar. 1953, L. Giovannoli leg. (AMNH). - San

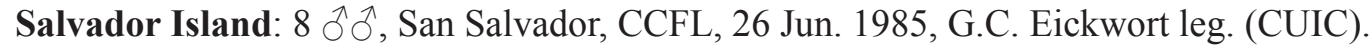

CAYMAN ISLANDS: Grand Cayman: 2 우, West Bay, Town Hall Cresent [sic], blacklight trap, 21 Jul.-1 Aug. 1986, D. Gicca leg. (NMNH).

The synonymy of the three species from Jamaica is based on an examination of the type series of each. The female of $L$.jamaicae is indistinguishable from that of $L$. gemmatum. The male holotype of $L$. bruesi is entirely pale, which is believed to be an artefact of preservation. It has identical collection information as the holotype of L. jamaicae and with exception of its colour matches male paratypes of that species.

\section{Key to Puerto Rican Lasioglossum}

1 Head and mesosoma brilliant metallic green to purple (subgenus Habralictellus) (Figs 2425); female T1 without appressed hairs on anterior surface (Fig. 27C); male inner metatibial spur pectinate; male genitalia without retrorse lobe (Fig 4C)

- Head and mesosoma dull metallic blue to golden green (subgenus Dialictus); female T1 with appressed hairs on anterior surface (Fig. 2C); male inner metatibial spur ciliate; male genitalia with retrorse lobe (Fig. 4A-B, D-F)

2 Face distinctly longer than broad (length/width ratio=1.04-1.06) (Figs 24A, 25A); three submarginal cells; mesoscutum densely punctate (Fig. 24C) ................ eickwortellum (Engel, 2001)

- Face distinctly wider than long (length/width ratio=0.87-0.88) (Figs 27A, 28A); two submarginal cells; mesoscutum sparsely punctate (Fig. 27C)

L. rufopanticis (Engel, 2001) 
3 Metasoma metallic; mesepisternum rugose; head relatively long (length/width ratio $=0.99-1.01$ ) (Figs 15A, 16A) ……............................................................................. ferrerii (Baker, 1906)

- Metasoma brown; mesepisternum punctate or if rugose then head wide (length/width ratio $=0.89-0.94)($ Figs 18A, 19A $)$

4 Mesepisternum smooth, sparsely punctate; female with dorsally interrupted T1 setal fan; male with long setose tufts on S4-S5 visible dorsally (Fig. 22D), S6 broadly emarginated

L. gundlachii (Baker, 1906)

- Mesepisternum punctate to rugose; female with complete T1 setal fan (Fig. 18C); male sterna not as above

5 Tegula enlarged, forming posterior angle (Figs 9C, 12C); mesoscutum finely punctate ............6

- Tegula ovoid (Fig. 2A); mesoscutum relatively coarsely punctate ................................................

6 Female supraclypeal area with punctures distinct; female propodeum with carinulate or rugulose dorsolateral slopes; female metasoma brown with pale apical margins; male mesepisternum

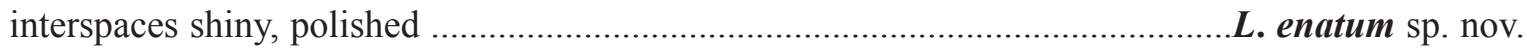

- Female supraclypeal area with punctures obscure; female propodeum with smooth-imbricate dorsolateral slopes; female metasoma entirely pale orange; male mesepisternum interspaces dull due to microsculpture (Mona Island) ……………............................................. monense sp. nov.

7 Female mesepisternum rugose, T1-T2 without tomentose patches basolaterally; male mesepisternum reticulate-rugose ........................................................................... genaroi sp. nov.

- Female mesepisternum punctate-reticulate, T1-T2 with tomentose patches basolaterally; male

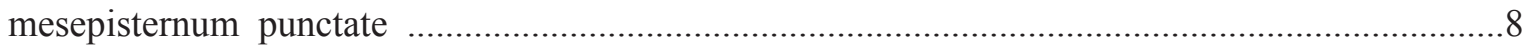

8 Female mesepisternum interspaces dull due to microsculpture; both sexes with dark tegula ........ .L. dispersum sp. nov.

- Female mesepisternum interspaces polished, shiny; both sexes with pale tegula (Mona Island) .. ..L. amona sp. nov.

\section{Discussion}

The bee fauna of the West Indies requires additional study, and halictid bees in particular remain poorly known. Much of our knowledge of the distribution and biology of halictid bees was summarized by Eickwort (1988), but thorough taxonomic studies are needed. This paper is the first attempt to revise all the Lasioglossum on an island in the Greater Antilles since Baker (1906) revised the Cuban species as the genus Chloralictus. A limited number of taxonomic studies on West Indian Lasioglossum have been published recently that are beginning to document the diversity of the fauna (Engel 2001b, 2011; Genaro 2001, 2016; Gibbs 2016). In a catalogue, Alayo (1976) documented ten undescribed species of Cuban Lasioglossum. Four of these, all ostensibly belonging to L. (Habralictellus), have now been described (Genaro 2001, 2016), but their subgeneric status remains in question due to the relatively substantial morphological differences between these Cuban species and other L. (Habralictellus). The discovery of five new species from Greater Puerto Rico and the correction of distribution records for three more species is relevant to understanding the conservation and historical biogeography of these bees. Furthermore, the description of the male of L. eickwortellum provides additional support for uniting members of the auratum and rufopanticis species groups within L. (Habralictellus).

Changing climate and rising sea-levels are likely to impact species endemic to islands (Lewsey et al. 2004; Wetzel et al. 2012). There are definite conservation implications for the restricted range and taxonomic limits of the species treated herein. For example, L. parvum is now believed to be largely 
restricted to Cuba rather than present on most islands in the Great Antilles. Although, none of the species of Lasioglossum in Puerto Rico is known to be in decline, there are insufficient data to make a proper assessment. Until this study, L. eickwortellum was known only from a single specimen and more than half the fauna was unknown to science. From the limited material available, some West Indian bees seem to have preferences for different altitudes within the islands (Figs 17, 20). Despite a number of historical collections from Puerto Rico, including the focused efforts of George Eickwort, nearly all the specimens of $L$. genaro $i$ sp. nov. examined come for a single survey of a small proportion of the island (Fig. 20). Species with preferences for low-elevation sites may be at risk from habitat loss due to human land-use and rising sea levels (Lewsey et al. 2004). However, high-elevation species are at greater risk, since species in montane ecosystems are more limited in their ability to move to suitable habitats (Hodkinson 2005).

From examination of material throughout the West Indies, it is clear that there are additional species in need of description from other islands (e.g., Gibbs 2016). As the number of species increases and the ranges of named taxa become better defined, it will become possible to understand the historical patterns of vicariance and dispersal that led to the current distribution of halictid bees in the West Indies. Additional surveys will be needed to capture uncommon species for molecular studies and to test species boundaries further. For example, Lasioglossum punctifrons, a species endemic to Dominica, has apparently not been captured since 1913 (Gibbs 2016), although it should be noted that sampling effort on the island has been limited. Efforts to recollect this species are warranted to determine its conservation status. The closely related species from Puerto Rico, L. eickwortellum, has also been quite rare in collections. Attempts to DNA barcode specimens of Lasioglossum ferrerii for this study were unsuccessful, so additional molecular-grade material is required. Two species putatively endemic to Mona Island are recognized, but additional study is needed to determine their status relative to related species on other islands. Molecular phylogenetic approaches would be valuable for determining the limits and validity of the subgenus $L$. (Habralictellus) and its relationship to other subgenera. Additional study to understand habitat preferences and nesting biology, and collections of material for population genetics and historical biogeography are needed.

\section{Acknowledgements}

This work would have been impossible without the generous loans of material used in this study. The following people made material available for examination: Brian Harris and Seán Brady (NMNH), Eli Wyman and Jerry Rozen (AMNH), Jason Dombroskie and Bryan Danforth (CUIC), Terry Griswold (BBSL, and his own loans from CMNH), Laurence Packer (PCYU), Alex Van Dam (UPRM), Kevin Williams (FSCA), Giar-Ann Kung and Brian Brown (LACM), David Notton (NHMUK), Jason Weintraub (ANSP), Sam Droege (PWRC), Jennifer Thomas and Michael Engel (SEMC), and Sara Prado (NCSU). Julio Genaro generously allowed me to visit his home in Toronto to examine specimens in his care and subsequently sent me specimens to examine. Laurence Packer arranged DNA barcoding of specimens. I thank the USDA-Specialty Crops Research Initiative and Project Integrated Crop Pollination (2012-51181-20105, Rufus Isaacs, PI) for post-doctoral funding. Images were taken with a system purchased with support from Project GREEEN, MSU AgBioResearch and a similar system at the J.B. Wallis / R.E. Roughley Museum of Entomology. Two anonymous reviewers provided useful comments on the manuscript.

\section{References}

Alayo P. 1973. Catálogo de los Himenopteros de Cuba. Editorial Pueblo y Educación, Havana, Cuba.

Alayo P. 1976. Introducción al estudio de los himenopteros de Cuba. Superfamilia Apoidea. Academía de Ciencias de Cuba, Instituto de Zoología, Serie Biológica 68: 1-41. 
Ascher J.S. \& Pickering J. 2016. Discover Life Bee Species Guide and World Checklist (Hymenoptera: Apoidea: Anthophila). Draft-45.

Available from http://www.discoverlife.org/mp/20q?guide=Apoidea_species [accessed 5 Jan. 2018].

Ashmead W.H. 1896. Notes on the Hymenoptera collected by the Bahamas Expedition from the State University of Iowa. Bulletin from the Laboratories of Natural History of the State University of Iowa 4: 28-32. Available from https://biodiversitylibrary.org/page/15404864 [accessed 5 Jan. 2018].

Ashmead W.H. 1900. Report upon the aculeate Hymenoptera of the islands of St. Vincent and Grenada, with additions to the parasitic Hymenoptera and a list of the described Hymenoptera of the West Indies. Transactions of the Entomological Society of London 2: 207-367.

https://doi.org/10.1111/j.1365-2311.1900.tb02379.x

Baker C.F. 1906. Halictinae de Cuba. In: Earle F.S. (ed.) Primer Informe de la Estacion Central Agronomica: 253-274. Ruiz y Hermano, Havana, Cuba.

Bivand R. \& Lewin-Koh N. 2017. maptools: Tools for reading and handling spatial objects, ver. 0.9-2. Available from https://cran.r-project.org/web/packages/maps/index.html [accessed 12 Jan. 2018].

Cockerell T.D.A. 1898. On some panurgine and other bees. Transactions of the American Entomological Society 25: 185-198.

Available from https://biodiversitylibrary.org/page/7522013 [accessed 5 Jan. 2018].

Cockerell T.D.A. 1904. New records of bees. The Entomologist 37: 231-236.

Available from https://biodiversitylibrary.org/page/25332701 [accessed 5 Jan. 2018].

Cockerell T.D.A. 1910. Some bees of the genus Augochlora from the West Indies. Proceedings of the United States National Museum 37: 489-497. https://doi.org/10.5479/si.00963801.37-1717.489

Cockerell T.D.A. 1912. Descriptions and records of bees.-XLVII. The Annals and Magazine of Natural History 10 (59): 484-493. https://doi.org/10.1080/00222931208693263

Cockerell T.D.A. 1915. Descriptions and records of bees.-LXVIII. The Annals and Magazine of Natural History 16 (91): 1-9. https://doi.org/10.1080/00222931508693679

Cresson E.T. 1865. On the Hymenoptera of Cuba. Proceedings of the Entomological Society of Philadelphia 4: 1-200.

Available from https://biodiversitylibrary.org/page/3783974 [accessed 5 Jan. 2018].

Cresson E.T. 1916. The Cresson types of Hymenoptera. Memoirs of the American Entomological Society 1: 1-141. Available from https://biodiversitylibrary.org/page/6513944 [accessed 5 Jan. 2018].

Dalla Torre C.G. 1896. Catalogus Hymenopterorum hucusque Descriptorum Systematicus et Synonymicus. Vol. 10. Apidae (Anthophila). Guillelmi Engelmann, Lipsiae [Leipzig].

Danforth B.N. 1999. Phylogeny of the bee genus Lasioglossum (Hymenoptera: Halictidae) based on mitochondrial COI sequence data. Systematic Entomology 24: 377-393.

https://doi.org/10.1046/j.1365-3113.1999.00087.x

Danforth B.N., Conway L. \& Ji S. 2003. Phylogeny of eusocial Lasioglossum reveals multiple losses of eusociality within a primitively eusocial clade of bees (Hymenoptera: Halictidae). Systematic Biology 52: 23-36. https://doi.org/10.1080/10635150390132687

Dereeper A., Guignon V., Blanc G., Audic S., Buffet S., Chevent F., Dufayard J.F., Guindon S., Lefort V., Lescot M., Claverie J.M. \& Gascuel O. 2008. Phylogeny.fr: robust phylogenetic analysis for the nonspecialist. Nucleic Acids Research 36: W465-W469. https://doi.org/10.1093/nar/gkn180

Dewitz H. 1881. Hymenopteren von Portorico. Berliner Entomologische Zeitschrift 25: 197-208. Available from https://biodiversitylibrary.org/page/9214321 [accessed 5 Jan. 2018]. 
Ducke A. 1902. Ein neues Subgenus von Halictus Latr. Zeitschrift für Systematische Hymenopterologie und Dipterologie 2: 102-103. Available from https://biodiversitylibrary.org/page/13367938 [accessed 5 Jan. 2018].

Edgar R.C. 2004. MUSCLE: multiple sequence alignment with high accuracy and high throughput. Nucleic Acids Research 32: 1792-1797. https://doi.org/10.1093/nar/gkh340

Eickwort G.C. 1988. Distribution patterns and biology of West Indian sweat bees (Hymenoptera: Halictidae). In: Liebherr J.K. (ed.) Zoogeography of Caribbean Insects: 232-253. Cornell University Press, Ithaca, New York.

Ellis M.D. 1914a. New bees of the genus Halictus (Hym.) from United States, Guatemala and Ecuador. Journal of the New York Entomological Society 22: 218-223.

Available from https://biodiversitylibrary.org/page/8188677 [accessed 5 Jan. 2018].

Ellis M.D. 1914b. New American bees of the genus Halictus (Hym.). Entomological News 25: 151-155.

Engel M.S. 2000. Classification of the bee tribe Augochlorini (Hymenoptera: Halictidae). Bulletin of the American Museum of Natural History 250: 1-89.

https://doi.org/10.1206/0003-0090(2000)250<0001:COTBTA >2.0.CO;2

Engel M.S. 2001a. A monograph of the Baltic amber bees and evolution of the Apoidea (Hymenoptera). Bulletin of the American Museum of Natural History 259: 1-192.

https://doi.org/10.1206/0003-0090(2001)259\%3C0001:AMOTBA\%3E2.0.CO;2

Engel M.S. 2001b. Three new Habralictellus bee species from the Caribbean (Hymenoptera: Halictidae). Solenodon 1: 33-37.

Engel M.S. 2011. A new species of Dialictus from Sombrero Island, Anguilla (Hymenoptera, Halictidae). ZooKeys 86: 61-68. https://doi.org/10.3897/zookeys.86.909

Friese H. 1902. Beitrag zur Apidenfauna der grossen Antillen (Hym). Zeitschrift für Systematische Hymenopterologie und Dipterologie 2: 196-201.

Available from https://biodiversitylibrary.org/page/13368040 [accessed 5 Jan. 2018].

Genaro J.A. 2001. Tres especies nuevas del genero Lasioglossum (Dialictus), grupo Habralictellus para Cuba (Hymenoptera: Halictidae). Solenodon 1: 38-44.

Genaro J.A. 2004. Las abejas de la Isla de la Juventud, Cuba (Hymenoptera: Apoidea). Boletín de la Sociedad Entomológica Aragonesa 34: 177-179.

Genaro J.A. 2007. Las abejas (Hymenoptera: Apoidea: Anthophila) de la Hispaniola, Antillas. Boletín de la Sociedad Entomológica Aragonesa 40: 247-254.

Genaro J.A. 2016. Especies nuevas y nuevos registros de abejas para las Antillas (Hymenoptera: Anthophila; Colletidae, Halictidae). Novitates Caribaea 10: 38-51.

Genaro J.A. \& Franz N.M. 2008. The bees of Greater Puerto Rico (Hymenoptera: Apoidea: Anthophila). Insecta Mundi 40: 1-27.

Gibbs J. 2009a. Integrative taxonomy identifies new (and old) species in the Lasioglossum (Dialictus) tegulare (Robertson) species group (Hymenoptera, Halictidae). Zootaxa 2032: 1-38.

Gibbs J.J. 2009b. New species in the Lasioglossum petrellum species group identified through an integrative taxonomic approach. The Canadian Entomologist 141: 371-396.

https://doi.org/10.4039/n09-020

Gibbs J. 2010a. Atypical wing venation in Dialictus and Hemihalictus and its implications for subgeneric classification of Lasioglossum. Psyche 2010: 1-6. https://doi.org/10.1155/2010/605390

Gibbs J. 2010b. Revision of the metallic species of Lasioglossum (Dialictus) in Canada (Hymenoptera, Halictidae, Halictini). Zootaxa 2591: 1-382. 
Gibbs J. 2011. Revision of the metallic Lasioglossum (Dialictus) of eastern North America (Hymenoptera: Halictidae: Halictini). Zootaxa 3073: 1-216.

Gibbs J. 2016. Bees of the family Halictidae Thomson, 1869 from Dominica, Lesser Antilles (Hymenoptera: Apoidea). European Journal of Taxonomy 180: 1-50.

https://doi.org/10.5852/ejt.2016.180

Gibbs J., Albert J. \& Packer L. 2012a. Dual origins of social parasitism in North American Dialictus (Hymenoptera: Halictidae) confirmed using a phylogenetic approach. Cladistics 28: 195-207.

https://doi.org/10.1111/j.1096-0031.2011.00373.x

Gibbs J., Brady S.G., Kanda K. \& Danforth B.N. 2012b. Phylogeny of halictine bees supports a shared origin of eusociality for Halictus and Lasioglossum (Apoidea: Anthophila: Halictidae). Molecular Phylogenetics and Evolution 65: 926-939. https://doi.org/10.1016/j.ympev.2012.08.013

Gibbs J., Packer L., Dumesh S. \& Danforth B.N. 2013. Revision and reclassification of Lasioglossum (Evylaeus), L. (Hemihalictus) and L. (Sphecodogastra) in eastern North America (Hymenoptera: Apoidea: Halictidae). Zootaxa 3672: 1-117. https://doi.org/10.11646/zootaxa.3672.1.1

Gowdey C.C. 1926. Catalogus insectorum Jamaicensis. Department of Agriculture Jamaica, Entomological Bulletin 4: 1-114.

Graenicher S. 1927. Bees of the genus Halictus from Miami, Florida. Psyche 34: 202-208. https://doi.org/10.1155/1927/75420

Gundlach J. 1887. Apuntes para la fauna Puerto-riqueña. Anales de la Sociedad Española de Historia Natural 15: 115-199.

Harris R.A. 1979. A glossary of surface sculpturing. Occasional Papers in Entomology 28: 1-31.

Hebert P.D.N., Penton E.H., Burns J.M., Janzen D.H. \& Hallwachs W. 2004. Ten species in one: DNA barcoding reveals cryptic species in the neotropical skipper butterfly Astraptes fulgerator. Proceedings of the National Academy of Sciences 101: 14812-14817. https://doi.org/10.1073/pnas.0406166101

Hodkinson I.D. 2005. Terrestrial insects along elevation gradients: species and community responses to altitude. Biological Reviews 80: 489-513. https://doi.org/10.1017/S1464793105006767

ICZN 1997. International Commission of Zoological Nomenclature. Opinion 1882: Dialictus Robertson, 1902 and Chloralictus Robertson, 1902 (Insecta, Hymenoptera): given precedence over Paralictus Robertson, 1901. Bulletin of Zoological Nomenclature 54: 201-202.

ICZN 1999. International Code of Zoological Nomenclature. $4^{\text {th }}$ ed. The International Trust for Zoological Nomenclature, London, UK. Available from http://www.iczn.org/iczn/index.jsp [accessed 5 Jan. 2018].

Krombein K.V. 1953. The wasps and bees of the Bimini Island group, Bahamas, British West Indies (Hymenoptera: Aculeata). American Museum Novitates 1633: 1-29.

Lewsey C., Cid G. \& Kruse E. 2004. Assessing climate change impacts on coastal infrastructure in the Eastern Caribbean. Marine Policy 28: 393-409. https://doi.org/10.1016/j.marpol.2003.10.016

Michener C.D. 1936. Some bees of the genus Halictus. Annals and Magazine of Natural History, Series 1018 (104): 281-287. https://doi.org/10.1080/00222933608655192

Michener C.D. 1974. The Social Behavior of the Bees. Belknap Press, Cambridge, Massachusetts.

Michener C.D. 1979. Biogeography of the bees. Annals of the Missouri Botanical Garden 66: 277. https://doi.org/10.2307/2398833

Michener C.D. 2007. The Bees of the World, $2^{\text {nd }}$ ed. The Johns Hopkins University Press, Baltimore, Maryland.

Moure J.S. 1947. Novos agrupamentos genéricos e algumas espécies novas de abelhas sulamericanas. Museu Paranaense Publicações Avulsas 3: 1-37. 
Moure J.S. 2001. Uma pequena abelha com cabeça e mandíbulas excepcionais (Hymenoptera, Halictidae). Revista Brasileira de Zoologia 18: 493-497.

Moure J.S. 2007. Halictini Thomson, 1869. In: Moure J.S., Urban D. \& Melo G.A.R. (eds) Catalogue of Bees (Hymenoptera, Apoidea) in the Neotropical Region: 823-870. Sociedade Brasileira de Entolologia, Curitiba, Brazil.

Moure J.S. \& Hurd P.D. 1982. On two new groups of Neotropical halictine bees (Hymenoptera, Apoidea). Dusenia 23: 46.

Moure J.S. \& Hurd P.D. 1987. An Annotated Catalog of the Halictid Bees of the Western Hemisphere (Hymenoptera: Halictidae). Smithsonian Institution Press, Washington, DC.

Pauly A. 1984. Classification des Halictidae de Madagascar et des îles voisines I. Halictinae (Hymenoptera Apoidea). Verhandlungen der Naturforschenden Gesellschaft in Basel 94: 121-156.

Pesenko Y.A. 2007. Subgeneric classification of the Palaearctic bees of the genus Evylaeus Robertson (Hymenoptera: Halictidae). Zootaxa 1500: 1-54.

R Core Team 2015. R: A language and environment for statistical computing. $\mathrm{R}$ version 3.2.2. R Foundation for Statistical Computing, Vienna. Available from https://www.R-project.org/ [accessed 5 Jan. 2018].

Ratnasingham S. \& Hebert P.D.N. 2007. bold: The Barcode of Life Data System(http://www.barcodinglife. org). Molecular Ecology Notes 7: 355-364. https://doi.org/10.1111/j.1471-8286.2007.01678.x

Raw A. 1985. The ecology of Jamaican bees (Hymenoptera). Revista Brasileira de Entomologia 29: $1-16$.

Robertson C. 1901. Some new and little-known bees. The Canadian Entomologist 33: 229-231. https://doi.org/10.4039/Ent33229-8

Robertson C. 1902a. Some new or little-known bees-II. The Canadian Entomologist 34: 48-49. https://doi.org/10.4039/Ent3448-2

Robertson C. 1902b. Synopsis of Halictinae. The Canadian Entomologist 34: 243-250. https://doi.org/10.4039/Ent34243-9

Rodríguez Velázquez D. \& Mestre Novoa N. 2002. Lista de los Collembola e Insecta (Coleoptera, Dermaptera, Dictyoptera, Mantodea, Diptera e Hymenoptera) de la Sierra de los Organos, Pinar del Río (Arthropoda: Hexapoda). Cocuyo 2: 6-10.

Sakagami S., Ebmer A.W. \& Tadauchi O. 1996. The halictine bees of Sri Lanka and the vicinity III. Sudila (Hymenoptera, Halictidae). Part 1. Esakia 36: 143-189.

Sandhouse G.A. 1923. The bee-genus Dialictus. The Canadian Entomologist 55: 193-195. https://doi.org/10.4039/Ent55193-8

Sandhouse G.A. 1924. New North American species belonging to the genus Halictus (Chloralictus). Proceedings of the United States National Museum 65 (19): 1-43.

Available from https://biodiversitylibrary.org/page/7767257 [accessed 5 Jan. 2018].

Schrottky C. 1911. Descripção de abelhas novas do Brazil e de regiões visinhas. Revista do Museo Paulista 8: 71-88.

Schwarz M.P., Richards M.H. \& Danforth B.N. 2007. Changing paradigms in insect social evolution: Insights from halictine and allodapine bees. Annual Review of Entomology 52: 127-150.

https://doi.org/10.1146/annurev.ento.51.110104.150950

Smith F. 1853. Catalogue of Hymenopterous Insects in the Collection of the British Museum. Part I. Andrenidae and Apidae. British Museum, London. 
Smith M.A., Bertrand C., Crosby K., Eveleigh E.S., Fernandez-Triana J., Fisher B.L., Gibbs J., Hajibabaei M., Hallwachs W., Hind K., Hrcek J., Huang D.-W., Janda M., Janzen D.H., Li Y., Miller S.E., Packer L., Quicke D., Ratnasingham S., Rodriguez J., Rougerie R., Shaw M.R., Sheffield C., Stahlhut J.K., Steinke D., Whitfield J., Wood M. \& Zhou X. 2012. Wolbachia and DNA barcoding insects: patterns, potential, and problems. PLoS One 7: e36514. https://doi.org/10.1371/journal.pone.0036514

Snelling R.R. 1993. Collecting on Guana Island, British Virgin Islands and Puerto Rico. Melissa 6: 5-6.

Snelling R.R. 2005. Wasps, ants and bees: aculeate Hymenoptera. In: Lazell J. (ed.) Island: Fact and Theory in Nature: 283-296. University of California Press, Berkeley, California.

Thomas R. \& Joglar R. 1996. The herpetology of Puerto Rico: Past, present, and future. Annals of the New York Academy of Sciences 776: 181-196. https://doi.org/10.1111/j.1749-6632.1996.tb17420.x

Warncke K. 1975. Beiträge zur Systematik und Verbreitung der Furchenbienen in der Türkei (Hymenoptera, Apoidea, Halictus). Polskie Pismo Entomologiczne 45: 81-123.

Wcislo W.T. 1997. Behavioral environments of sweat bees (Halictinae) in relation to variability in social organization. In: Choe J.C. \& Crespi B.J. (eds) The Evolution of Social Behaviour in Insects and Arachnids: 316-332. Cambridge University Press, Cambridge, New York.

Wetzel F.T., Kissling W.D., Beissmann H. \& Penn D.J. 2012. Future climate change driven sea-level rise: secondary consequences from human displacement for island biodiversity. Global Change Biology 18: 2707-2719. https://doi.org/10.1111/j.1365-2486.2012.02736.x

Wickham H. 2009. ggplot2: Elegant Graphics for Data Analysis. Springer, New York.

Wolcott G.N. 1948. The insects of Puerto Rico. The Journal of Agriculture of the University of Puerto Rico 32: 749-975.

Yanega D. 1997. Demography and sociality in halictine bees (Hymenoptera: Halictidae). In: Choe J.C. \& Crespi B.J. (eds) The Evolution of Social Behaviour in Insects and Arachnids: 293-315. Cambridge University Press, Cambridge, New York.

Zink R.M., Groth J.G., Vázquez-Miranda H. \& Barrowclough G.F. 2016. Geographic variation, null hypotheses, and subspecies limits in the California gnatcatcher: A response to McCormack and Maley. The Auk 133: 59-68. https://doi.org/10.1642/AUK-15-63.1

Manuscript received: 26 June 2017

Manuscript accepted: 1 August 2017

Published on: 1 February 2018

Topic editor: Gavin Broad

Desk editor: Danny Eibye-Jacobsen

Printed versions of all papers are also deposited in the libraries of the institutes that are members of the EJT consortium: Muséum national d'Histoire naturelle, Paris, France; Botanic Garden Meise, Belgium; Royal Museum for Central Africa, Tervuren, Belgium; Natural History Museum, London, United Kingdom; Royal Belgian Institute of Natural Sciences, Brussels, Belgium; Natural History Museum of Denmark, Copenhagen, Denmark; Naturalis Biodiversity Center, Leiden, the Netherlands; Museo Nacional de Ciencias Naturales-CSIC, Madrid, Spain; Real Jardín Botánico de Madrid CSIC, Spain. 